\title{
Unified correspondence as a proof-theoretic tool
}

GIUSEPPE GRECO, Faculty of Technology, Policy and Management, Delft University of Technology, Jaffalaan 5, 2628 BX, Delft, the Netherlands. E-mail:G.Greco@tudelft.nl

MINGHUI MA, Institute for Logic and Intelligence, Southwest University, Tiansheng Road No.2, Beibei, Chongqing, 400715, China.

E-mail:mmh.thu@gmail.com

ALESSANDRA PALMIGIANO, Faculty of Technology, Policy and Management, Delft University of Technology, Jaffalaan 5, 2628 BX, Delft, the Netherlands and Department of Pure and Applied Mathematics, University of Johannesburg, PO Box 524, Auckland Park 2006, South Africa.

E-mail:A.Palmigiano@tudelft.nl

APOSTOLOS TZIMOULIS AND ZHIGUANG ZHAO, Faculty of Technology, Policy and Management, Delft University of Technology, Jaffalaan 5, 2628 BX, Delft, the Netherlands.

E-mail: A.Tzimoulis-1@tudelft.nl; Z.Zhao-3@tudelft.nl

\begin{abstract}
The present article aims at establishing formal connections between correspondence phenomena, well known from the area of modal logic, and the theory of display calculi, originated by Belnap. These connections have been seminally observed and exploited by Marcus Kracht, in the context of his characterization of the modal axioms (which he calls primitive formulas) which can be effectively transformed into 'analytic' structural rules of display calculi. In this context, a rule is 'analytic' if adding it to a display calculus preserves Belnap's cut-elimination theorem. In recent years, the state-of-the-art in correspondence theory has been uniformly extended from classical modal logic to diverse families of non-classical logics, ranging from (bi-)intuitionistic (modal) logics, linear, relevant and other substructural logics, to hybrid logics and mu-calculi. This generalization has given rise to a theory called unified correspondence, the most important technical tools of which are the algorithm ALBA, and the syntactic characterization of Sahlqvist-type classes of formulas and inequalities which is uniform in the setting of normal DLE-logics (logics the algebraic semantics of which is based on bounded distributive lattices). We apply unified correspondence theory, with its tools and insights, to extend Kracht's results and prove his claims in the setting of DLE-logics. The results of the present article characterize the space of properly displayable DLE-logics.
\end{abstract}

Keywords: Display calculi, unified correspondence, distributive lattice expansions, properly displayable logics.

\section{Introduction}

The present article applies the results and insights of unified correspondence theory [16] to establish formal connections between correspondence phenomena, well known from the area of modal logic, and the theory of display calculi, introduced by Belnap [2]. 
Sahlqvist correspondence theory: Sahlqvist theory [49] is among the most celebrated and useful results of the classical theory of modal logic, and one of the hallmarks of its success. It provides an algorithmic, syntactic identification of a class of modal formulas whose associated normal modal logics are strongly complete with respect to elementary (i.e. first-order definable) classes of frames.

Unified correspondence. In recent years, building on duality-theoretic insights [21], an encompassing perspective has emerged which has made it possible to export the state-of-the-art in Sahlqvist theory from modal logic to a wide range of logics which includes, among others, intuitionistic and distributive lattice-based (normal modal) logics [18], non-normal (regular) modal logics [46], substructural logics [20], hybrid logics [24] and mu-calculus [12-14].

The breadth of this work has stimulated many and varied applications. Some are closely related to the core concerns of the theory itself, such as the understanding of the relationship between different methodologies for obtaining canonicity results $[19,45]$, or of the phenomenon of pseudocorrespondence [22]. Other, possibly surprising applications include the dual characterizations of classes of finite lattices [32] and the epistemic interpretation of modalities on RS-frames [15]. Finally, the insights of unified correspondence theory have made it possible to determine the extent to which the Sahlqvist theory of classes of normal Distributive Lattice Expansions (DLEs) can be reduced to the Sahlqvist theory of normal Boolean expansions, by means of Gödel-type translations [23]. These and other results have given rise to a theory called unified correspondence [16].

Tools of unified correspondence theory. The most important technical tools in unified correspondence are: (i) a very general syntactic definition of the class of Sahlqvist formulas, which applies uniformly to each logical signature and is given purely in terms of the order-theoretic properties of the algebraic interpretations of the logical connectives; (ii) the Ackermann Lemma Based Algorithm (ALBA), which effectively computes first-order correspondents of input term-inequalities, and is guaranteed to succeed on a wide class of inequalities (the so-called inductive inequalities) which, like the Sahlqvist class, can be defined uniformly in each mentioned signature, and which properly and significantly extends the Sahlqvist class.

Unified correspondence and display calculi. The present article aims at applying the tools of unified correspondence to address the identification of the syntactic shape of axioms which can be translated into analytic structural rules ${ }^{1}$ of a display calculus, and the definition of an effective procedure for transforming axioms into such rules. In recent years, these questions have been intensely investigated in the context of various proof-theoretic formalisms (cf. [7, 8, 11, 37, 39-41, 43, 44]). Perhaps the first paper in this line of research is [38], which addresses these questions in the setting of display calculi for basic normal modal and tense logic. Interestingly, in [38], the connections between Sahlqvist theory and display calculi started to be observed, but have not been systematically explored there nor (to the knowledge of the authors) in subsequent papers in the same research line.

Contributions. The two tools of unified correspondence can be put to use to generalize Kracht's transformation procedure from axioms into analytic rules. This generalization concerns more than one aspect. First, in the same way in which the definitions of Sahlqvist and inductive inequalities can be given uniformly in each logical signature, the definition of primitive formulas/inequalities is introduced for any logical framework the algebraic semantics of which is based on distributive

\footnotetext{
${ }^{1}$ Analytic rules (cf. Definition 4) are those which can be added to a proper display calculus (cf. Section 2.2) obtaining another proper display calculus.
} 
lattices with operators (these will be referred to as DLE-logics, (cf. Definition 10 and Footnote 13 for terminology). Secondly, in the context of each such logical framework, we introduce a hierarchy of subclasses of inductive inequalities, progressively extending the primitive inequalities, the largest of which is the class of so-called analytic inductive inequalities. This is a syntactic generalization of the class of primitive formulas/inequalities. We provide an effective procedure, based on ALBA, which transforms each analytic inductive inequality into an equivalent set of analytic rules. Moreover, we show that any analytic rule can be effectively and equivalently transformed into some analytic inductive inequality. Finally, we show that any analytic rule can be effectively and equivalently transformed into one of a particularly nice shape, collectively referred to as special rules.

Structure of the article. In Section 2, preliminaries on display calculi are collected. In Section 3, the setting of basic DLE-logics is introduced, and the algorithm ALBA for them. In Section 4, the display calculi DL and DL* for DLE-logics are introduced, and their basic properties are proven. In Section 5, Kracht's notion of primitive formulas is generalized to primitive inequalities in each DLElanguage, as well as their connection with special structural rules for display calculi (cf. Definition 6). It is also shown that, for any language $\mathcal{L}_{\mathrm{DLE}}$, each primitive $\mathcal{L}_{\mathrm{DLE}}$-inequality is equivalent on perfect $\mathcal{L}_{\mathrm{DLE}}$-algebras to a set of special structural rules in the language of the associated display calculus DL, and that the validity of each such special structural rule is equivalent to the validity of

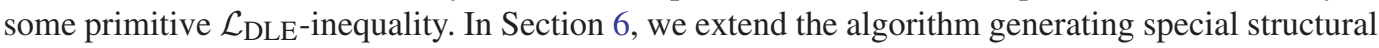
rules in the language of DL from input primitive $\mathcal{L}_{\mathrm{DLE}}$-inequalities to a hierarchy of classes of non-primitive $\mathcal{L}_{\mathrm{DLE}}$-inequalities, the most general of which is referred to as restricted analytic inductive inequalities (cf. Definition 51). Our procedure for obtaining this extension makes use of ALBA to equivalently transform any restricted analytic inductive $\mathcal{L}_{\mathrm{DLE}}$-inequality into one or more primitive $\mathcal{L}_{\mathrm{DLE}}^{*}$-inequalities. In Section 7 , the class of restricted analytic inductive inequalities is further extended to the analytic inductive inequalities (cf. Definition 55). Each analytic inductive inequality can be equivalently transformed into some analytic rule of a restricted shape, captured in the notion of quasi-special structural rule (cf. Definition 8) in the language of DL. Once again, the key step of the latter procedure makes use of ALBA, this time to equivalently transform any analytic inductive inequality into one or more suitable quasi-inequalities in $\mathcal{L}_{\mathrm{DLE}}^{*}$. We also show that each analytic rule is equivalent to some analytic inductive inequality. This back-and-forth correspondence between analytic rules and analytic inductive inequalities characterizes the space of properly displayable DLElogics as the axiomatic extensions of the basic DLE-logic obtained by means of analytic inductive inequalities. In Section 8 , we show that for any language $\mathcal{L}_{\text {DLE }}$, any properly displayable DLE-logic is specially displayable, which implies that any properly displayable $\mathcal{L}_{\mathrm{DLE}}^{*}$-logic can be axiomatized by means of primitive $\mathcal{L}_{\mathrm{DLE}}^{*}$-inequalities. This last result generalizes an analogous statement made by Kracht in the setting of properly displayable tense modal logics, which was proven in $[9,10]$ in the same setting. In Section 9, we outline a comparison between the present treatment and that of $[9,10]$. In Section 10, we present our conclusions. Various proofs are collected in Appendices A-D.

\section{Preliminaries on display calculi}

In the present section, we provide an informal introduction to the main features of display calculi without any attempt at being self-contained. We refer the reader to [56] for an expanded treatment. Our presentation follows [29, Section 2.2].

Display calculi are among the approaches in structural proof theory aimed at the uniform development of an inferential theory of meaning of logical constants (logical connectives) aligned 
with the principles of proof-theoretic semantics [50, 51]. Display calculi have been successful in giving adequate proof-theoretic semantic accounts of logics—such as certain modal and substructural logics [35], and more recently also Dynamic Epistemic Logic [30] and PDL [27]—which have notoriously been difficult to treat with other approaches. Here we mainly report and elaborate on the work of Belnap [2], Wansing [56], Goré [34, 35] and Restall [48].

\subsection{Belnap's display logic}

Nuel Belnap introduced the first display calculus, which he calls Display Logic [2], as a sequent system augmenting and refining Gentzen's basic observations on structural rules. Belnap's refinement is based on the introduction of a special syntax for the constituents of each sequent. Indeed, his calculus treats sequents $X \vdash Y$ where $X$ and $Y$ are so-called structures, i.e. syntactic objects inductively defined from formulas using an array of special meta-logical connectives. Belnap's basic idea is that, in the standard Gentzen formulation, the comma symbol ',' separating formulas in the precedent and in the succedent of sequents can be recognized as a metalinguistic connective, the behaviour of which is defined by the structural rules.

Belnap took this idea further by admitting not only the comma, but also other meta-logical connectives to build up structures out of formulas, and called them structural connectives. Just like the comma in standard Gentzen sequents is interpreted contextually (i.e. as conjunction when occurring on the left-hand side and as disjunction when occurring on the right-hand side), each structural connective typically corresponds to a pair of logical connectives, and is interpreted as one or the other of them contextually (more of this in Section 4.2). Structural connectives maintain relations with one another, the most fundamental of which take the form of adjunctions and residuations. These relations make it possible for the calculus to enjoy the powerful property which gives it its name, namely, the display property. Before introducing it formally, let us agree on some auxiliary definitions and nomenclature: structures are defined much in the same way as formulas, taking formulas as atomic components and closing under the given structural connectives; therefore, each structure can be uniquely associated with a generation tree. Every node of such a generation tree defines a substructure. A sequent $X \vdash Y$ is a pair of structures $X, Y$. The display property, stated similarly to the one below, appears in [2, Theorem 3.2]:

\section{DEFINITION 1}

A proof system enjoys the display property iff for every sequent $X \vdash Y$ and every substructure $Z$ of either $X$ or $Y$, the sequent $X \vdash Y$ can be equivalently transformed, using the rules of the system, into a sequent which is either of the form $Z \vdash W$ or of the form $W \vdash Z$, for some structure $W$. In the first case, $Z$ is displayed in precedent position, and in the second case, $Z$ is displayed in succedent position. ${ }^{2}$ The rules enabling this equivalent rewriting are called display postulates.

Thanks to the fact that display postulates are semantically based on adjunction and residuation, exactly one of the two alternatives mentioned in the definition above can soundly occur. In other words, in a calculus enjoying the display property, any substructure of any sequent $X \vdash Y$ is always displayed either only in precedent position or only in succedent position. This is why we can talk about occurrences of substructures in precedent or in succedent position, even if they are nested deep within a given sequent, as illustrated in the following example which is based on the display

\footnotetext{
${ }^{2}$ In the following sections, we will find it useful to differentiate between the full and the relativized display property (cf. discussion before Proposition 22).
} 
postulates between the structural connectives ; and >:

$\frac{\frac{Y \vdash X>Z}{X ; Y \vdash Z}}{\frac{Y ; X \vdash Z}{X \vdash Y>Z}}$

In the derivation above, the structure $X$ is on the right side of the turnstile, but it is displayable on the left, and, therefore, is in precedent position. The display property is a crucial technical ingredient for Belnap's cut-elimination metatheorem: for instance, it provides the core mechanism for the satisfaction of the crucial condition $\mathrm{C}_{8}$, discussed in the following subsection.

\subsection{Proper display calculi and canonical cut-elimination}

In [2], a metatheorem is proven, which gives sufficient conditions in order for a sequent calculus to enjoy cut-elimination. ${ }^{3}$ This metatheorem captures the essentials of the Gentzen-style cut-elimination procedure, and is the main technical motivation for the design of Display Logic. Belnap's metatheorem gives a set of eight conditions on sequent calculi, which are relatively easy to check, since most of them are verified by inspection on the shape of the rules. Together, these conditions guarantee that the cut is eliminable in the given sequent calculus, and that the calculus enjoys the subformula property. When Belnap's metatheorem can be applied, it provides a much smoother and more modular route to cut-elimination than the Gentzen-style proofs. Moreover, as we will see later, a Belnap style cut-elimination theorem is robust with respect to adding a general class of structural rules, and with respect to adding new logical connectives, whereas a Gentzen-style cutelimination proof for the modified system cannot be deduced from the old one, but must be proved from scratch.

In a slogan, we could say that Belnap-style cut-elimination is to ordinary cut-elimination what canonicity is to completeness: indeed, canonicity provides a uniform strategy to achieve completeness. In the same way, the conditions required by Belnap's metatheorem ensure that one and the same given set of transformation steps is enough to achieve Gentzen-style cut-elimination for any system satisfying them.

In what follows, we review and discuss eight conditions which are stronger in certain respects than those in [2], ${ }^{4}$ and which define the notion of proper display calculus in [56]. ${ }^{5}$

$\mathbf{C}_{1}$ : Preservation of formulas. This condition requires each formula occurring in a premise of a given inference to be the subformula of some formula in the conclusion of that inference. That is, structures may disappear, but not formulas. This condition is not included in the list of sufficient conditions of the cut-elimination metatheorem, but, in the presence of cut-elimination, it guarantees the subformula property of a system. Condition $\mathrm{C}_{1}$ can be verified by inspection on the shape of the rules. In practice, Condition $\mathrm{C}_{1}$ bans rules in which structure variables occurring in some premise to not occur also in the conclusion, since in concrete derivations these are typically instantiated with (structures containing) formulas which would then disappear in the application of the rule.

\footnotetext{
${ }^{3}$ As Belnap observed on page 389 in [2]: 'The eight conditions are supposed to be a reminiscent of those of Curry' in [25].

${ }^{4}$ See also $[3,48]$ and the 'second formulation' of condition C6/7 in Section 4.4 of [56].

${ }^{5}$ See the 'first formulation' of conditions C6, C7 in Section 4.1 of [56].
} 
$\mathbf{C}_{2}$ : Shape-alikeness of parameters. This condition is based on the relation of congruence between parameters (i.e. non-active parts) in inferences; the congruence relation is an equivalence relation which is meant to identify the different occurrences of the same formula or substructure along the branches of a derivation [2, Section 4], [48, Definition 6.5]. Condition $\mathrm{C}_{2}$ requires that congruent parameters be occurrences of the same structure. This can be understood as a condition on the design of the rules of the system if the congruence relation is understood as part of the specification of each given rule; i.e. each schematic rule of the system comes with an explicit specification of which elements are congruent to which (and then the congruence relation is defined as the reflexive and transitive closure of the resulting relation). In this respect, $\mathrm{C}_{2}$ is nothing but a sanity check, requiring that the congruence is defined in such a way that indeed identifies the occurrences which are intuitively 'the same'. 6

$\mathbf{C}_{3}$ : Non-proliferation of parameters. Like the previous one, also this condition is actually about the definition of the congruence relation on parameters. Condition $\mathrm{C}_{3}$ requires that, for every inference (i.e. rule application), each of its parameters is congruent to at most one parameter in the conclusion of that inference. Hence, the condition stipulates that for a rule such as the following,

$$
\frac{X \vdash Y}{X, X \vdash Y}
$$

the structure $X$ from the premise is congruent to only one occurrence of $X$ in the conclusion sequent. Indeed, the introduced occurrence of $X$ should be considered congruent only to itself. Moreover, given that the congruence is an equivalence relation, condition $\mathrm{C}_{3}$ implies that, within a given sequent, any substructure is congruent only to itself. In practice, in the general schematic formulation of rules, we will use the same structure variable for two different parametric occurrences if and only if they are congruent, so a rule such as the one above is de facto banned.

\section{REMARK 2}

Conditions $\mathrm{C}_{2}$ and $\mathrm{C}_{3}$ make it possible to follow the history of a formula along the branches of any given derivation. In particular, $\mathrm{C}_{3}$ implies that the the history of any formula within a given derivation has the shape of a tree, which we refer to as the history-tree of that formula in the given derivation. Notice, however, that the history-tree of a formula might have a different shape than the portion of the underlying derivation corresponding to it; for instance, the following application of the Contraction rule gives rise to a bifurcation of the history-tree of $A$ which is absent in the underlying branch of the derivation tree, given that Contraction is a unary rule.

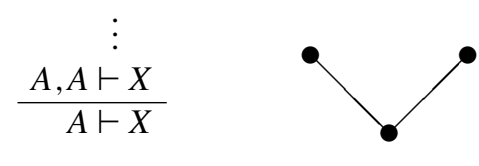

\footnotetext{
${ }^{6}$ Our convention throughout the article is that congruent parameters are denoted by the same letter. For instance, in the rule

$$
\frac{X ; Y \vdash Z}{Y ; X \vdash Z}
$$

the structures $X, Y$ and $Z$ are parametric and the occurrences of $X$ (resp. $Y, Z$ ) in the premise and the conclusion are congruent.
} 
$\mathbf{C}_{4}$ : Position-alikeness of parameters. This condition bans any rule in which a (sub)structure in precedent (resp. succedent) position in a premise is congruent to a (sub)structure in succedent (resp. precedent) position in the conclusion.

$\mathbf{C}_{5}$ : Display of principal constituents. This condition requires that any principal occurrence (i.e. a non-parametric formula occurring in the conclusion of a rule application, cf. [2, Condition C5]) be always either the entire antecedent or the entire consequent part of the sequent in which it occurs. In the following section, a generalization of this condition will be discussed, in view of its application to the main focus of interest of the present chapter.

The following conditions $\mathrm{C}_{6}$ and $\mathrm{C}_{7}$ are not reported below as they are stated in the original paper [2], but as they appear in [56, Section 4.1].

$\mathbf{C}_{6}$ : Closure under substitution for succedent parameters. This condition requires each rule to be closed under simultaneous substitution of arbitrary structures for congruent formulas which occur in succedent position. Condition $\mathrm{C}_{6}$ ensures, for instance, that if the following inference is an application of the rule $R$ :

$$
\frac{(X \vdash Y)\left([A]_{i}^{\mathrm{suc}} \mid i \in I\right)}{\left(X^{\prime} \vdash Y^{\prime}\right)[A]^{\text {suc }}} R
$$

and $\left([A]_{i}^{\text {suc }} \mid i \in I\right)$ represents all and only the occurrences of $A$ in the premiss which are congruent to the occurrence of $A$ in the conclusion, ${ }^{7}$ then also the following inference is an application of the same rule $R$ :

$$
\frac{(X \vdash Y)\left([Z / A]_{i}^{\mathrm{suc}} \mid i \in I\right)}{\left(X^{\prime} \vdash Y^{\prime}\right)[Z / A]^{\mathrm{suc}}} R
$$

where the structure $Z$ is substituted for $A$.

This condition caters for the step in the cut-elimination procedure in which the cut needs to be 'pushed up' over rules in which the cut-formula in succedent position is parametric. Indeed, condition $\mathrm{C}_{6}$ guarantees that, in the picture below, a well-formed subtree $\pi_{1}[Y / A]$ can be obtained from $\pi_{1}$ by replacing any occurrence of $A$ corresponding to a node in the history tree of the cut-formula $A$ by $Y$, and hence the following transformation step is guaranteed go through uniformly and 'canonically':

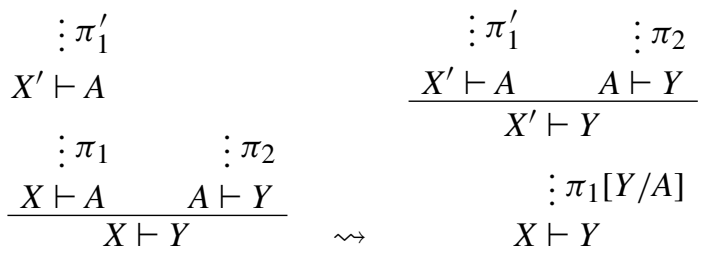

if each rule in $\pi_{1}$ verifies condition $\mathrm{C}_{6}$.

\footnotetext{
${ }^{7}$ Clearly, if $I=\varnothing$, then the occurrence of $A$ in the conclusion is congruent to itself.
} 
$\mathbf{C}_{7}$ : Closure under substitution for precedent parameters. This condition requires each rule to be closed under simultaneous substitution of arbitrary structures for congruent formulas which occur in precedent position. Condition $\mathrm{C}_{7}$ can be understood analogously to $\mathrm{C}_{6}$, relative to formulas in precedent position. Therefore, for instance, if the following inference is an application of the rule $R$ :

$$
\frac{(X \vdash Y)\left([A]_{i}^{\text {pre }} \mid i \in I\right)}{\left(X^{\prime} \vdash Y^{\prime}\right)[A]^{\text {pre }}} R
$$

then also the following inference is an instance of $R$ :

$$
\frac{(X \vdash Y)\left([Z / A]_{i}^{\text {pre }} \mid i \in I\right)}{\left(X^{\prime} \vdash Y^{\prime}\right)[Z / A]^{\text {pre }}} R
$$

Similarly to what has been discussed for condition $\mathrm{C}_{6}$, condition $\mathrm{C}_{7}$ caters for the step in the cutelimination procedure in which the cut needs to be 'pushed up' over rules in which the cut-formula in precedent position is parametric.

$\mathbf{C}_{8}$ : Eliminability of matching principal constituents. This condition requests a standard Gentzenstyle checking, which is now limited to the case in which both cut-formulas are principal, i.e. each of them has been introduced with the last rule application of each corresponding subdeduction. In this case, analogously to the proof Gentzen-style, condition $\mathrm{C}_{8}$ requires being able to transform the given deduction into a deduction with the same conclusion in which either the cut is eliminated altogether, or is transformed in one or more applications of cut involving proper subformulas of the original cut-formulas.

THEOREM 3 (cf. [57]Section 3.3, Appendix A)

Any calculus satisfying conditions $\mathrm{C}_{2}, \mathrm{C}_{3}, \mathrm{C}_{4}, \mathrm{C}_{5}, \mathrm{C}_{6}, \mathrm{C}_{7}, \mathrm{C}_{8}$ enjoys cut-elimination. If $\mathrm{C}_{1}$ is also satisfied, then the calculus enjoys the subformula property.

Rules introducing logical connectives. In display calculi, these rules, sometimes referred to as operational or logical rules as opposed to structural rules, typically occur in two flavors: operational rules which translate one structural connective in the premises in the corresponding connective in the conclusion, and operational rules in which both the operational connective and its structural counterpart are introduced in the conclusion. An example of this pattern is provided below for the case of the modal operator 'diamond':

$$
\frac{\circ A \vdash X}{\diamond A \vdash X} \diamond_{L} \quad \frac{X \vdash A}{\circ X \vdash \diamond A} \diamond_{R}
$$

In Section 4, this introduction pattern will be justified from a semantic viewpoint and generalized to logical connectives of arbitrary arity and polarity of their coordinates. From this example, it is clear that the introduction rules capture the rock bottom behaviour of the logical connective in question; additional properties (for instance, normality, in the case in point), which might vary depending on the logical system, are to be captured at the level of additional (purely structural) rules. This enforces a clear-cut division of labour between operational rules, which only encode the basic prooftheoretic meaning of logical connectives, and structural rules, which account for all extra relations and properties, and which can be modularly added or removed, thus accounting for the space of 
axiomatic extensions of a given base logic. Besides being important from the viewpoint of a prooftheoretic semantic account of logical connectives, this neat division of labour is also key to the research programme in proof theory aimed at developing more robust versions of Gentzen's cutelimination theory. Indeed, as we have seen, Belnap's strategy in this respect precisely pivots on the identification of conditions (mainly on the structural rules of a display calculus) which guarantee that structural rules satisfying them can be safely added in a modular fashion to proper display calculi without disturbing the canonical cut-elimination. In the following subsection, we will expand on the consequences of these conditions on the design of structural rules. Specifically, we report on three general shapes of structural rules. Identifying axioms or formulas which can be effectively translated into rules of one of these shapes is the main goal of the present article.

\subsection{Analytic, special and quasi-special structural rules}

In the remainder of the article, we will adopt the following convention regarding structural variables and terms: variables $X, Y, Z, W$ denote structures, and so do $S, T, U, V$. However, when describing rule schemas in abstract terms, we will often write e.g. $X \vdash S$, and in this context we understand that $X, Y, Z, W$ denote structure variables actually occurring in the given rule scheme, whereas $S, T, U, V$ are used as meta-variables for (possibly) compound structural terms such as $X ; Y$.

DEFINITION 4 (Analytic structural rules)

(cf. [10, Definition 3.13]) A structural rule which satisfies conditions $\mathrm{C}_{1}-\mathrm{C}_{7}$ is an analytic structural rule.

Clearly, adding analytic structural rules to a proper display calculus (cf. Section 2.2) yields a proper display calculus.

\section{REMARK 5}

In the setting of calculi with the relativized display property, ${ }^{8}$ if a given analytic structural rule $\rho$ can be applied in concrete derivations of the calculus then $\rho$ is interderivable, modulo applications of display postulates, with a rule of the following form:

$$
\frac{\left(S_{j}^{i} \vdash Y^{i} \mid 1 \leq i \leq n \text { and } 1 \leq j \leq n_{i}\right) \quad\left(X^{k} \vdash T_{\ell}^{k} \mid 1 \leq k \leq m \text { and } 1 \leq \ell \leq m_{k}\right)}{(S \vdash T)\left[Y^{i}\right]^{\text {suc }}\left[X^{k}\right]^{\text {pre }}}
$$

where $X^{k}$ (resp. $Y^{i}$ ) might occur in $S_{j}^{i}$ or in $T_{\ell}^{k}$ in precedent (resp. succedent) position for some $i, j, k, \ell$ and moreover, $X^{k}$ and $Y^{j}$ occur exactly once in $S \vdash T$ in precedent and succedent position, respectively, for all $j, k$.

The most common analytic rules occur in the following proper subclass:

DEFINITION 6 (Special structural rules)

(cf. [38, Section 5, discussion after Theorem 15]) Special structural rules are analytic structural rules of one of the following forms:

$$
\frac{\left(X \vdash T_{i} \mid 1 \leq i \leq n\right)}{X \vdash T} \quad \frac{\left(S_{i} \vdash Y \mid 1 \leq i \leq n\right)}{S \vdash Y}
$$

where $X$ (resp. $Y$ ) does not occur in any $T_{i}$ (resp. $S_{i}$ ) for $1 \leq i \leq n$ nor in $T$ (resp. $S$ ).

\footnotetext{
${ }^{8} \mathrm{Cf}$. discussion before Proposition 22
} 
In [38], Kracht establishes a correspondence between special rules and primitive formulas in the setting of tense modal logic, which will be generalized in Section 5.1 below.

REMARK 7

An alternative way to define special rules, which would also be perhaps more in line with the spirit of display calculi, would be as those rules

$$
\frac{\left(S_{i} \vdash T_{i} \mid 1 \leq i \leq n\right)}{S \vdash T}
$$

such that some variable $X$ occurs exactly once in each premise and in the conclusion, and always in the same (antecedent or consequent) position. In this way, the class of special rules would be closed under application of display postulates. Applying the general procedure described in Section 7.1 to primitive inequalities (cf. Definition 28) always yields special rules in the less restrictive sense here specified, but not in the sense of Definition 6 above. This fact might be taken as a motivation for adopting the less restrictive definition. However, the more restrictive definition can be immediately verified of a concrete rule, which is the reason why we prefer it over the less restricted one.

In [38], Kracht states without proof that any analytic structural rules in the language of classical tense logic $K t$ is equivalent to some special structural rule. Kracht's claim has been proved with model-theoretic techniques in [10,47]. In Section 8, we generalize these results using ALBA from classical tense logic to arbitrary DLE-logics. The following definition is instrumental in achieving this generalization:

DEFINITION 8 (Quasi-special structural rules) Quasi-special structural rules are analytic structural rules of the following form:

$$
\frac{\left(S_{j}^{i} \vdash Y^{i} \mid 1 \leq i \leq n \text { and } 1 \leq j \leq n_{i}\right) \quad\left(X^{k} \vdash T_{\ell}^{k} \mid 1 \leq k \leq m \text { and } 1 \leq \ell \leq m_{k}\right)}{(S \vdash T)\left[Y^{i}\right]^{\text {suc }}\left[X^{k}\right]^{\text {pre }}}
$$

where $X^{k}$ and $Y^{i}$ do not occur in any $S_{j}^{i}, T_{\ell}^{k}$ (and occur in $S \vdash T$ exactly once).

\section{Preliminaries on DLE-logics and ALBA}

In the present section, we collect preliminaries on logics for distributive lattice expansions (or DLElogics), reporting in particular on their language, axiomatization and algebraic semantics. Then we report on the definition of inductive DLE-inequalities, and outline, without any attempt at being self-contained, the algorithm ALBA $^{9}$ (cf. $[16,18]$ ) for each DLE-language.

\subsection{Syntax and semantics for DLE-logics}

Our base language is an unspecified but fixed language $\mathcal{L}_{\text {DLE }}$, to be interpreted over distributive lattice expansions of compatible similarity type. This setting uniformly accounts for many well-known logical systems, such as distributive and positive modal logic, intuitionistic and bi-intuitionistic (modal) logic, tense logic, and (distributive) full Lambek calculus.

\footnotetext{
${ }^{9} \mathrm{ALBA}$ is the acronym of Ackermann Lemma Based Algorithm.
} 
In our treatment, we will make heavy use of the following auxiliary definition: an order type over $n \in \mathbb{N}^{10}$ is an $n$-tuple $\varepsilon \in\{1, \partial\}^{n}$. For every order type $\varepsilon$, we denote its opposite order type by $\varepsilon^{\partial}$, i.e. $\varepsilon_{i}^{\partial}=1$ iff $\varepsilon_{i}=\partial$ for every $1 \leq i \leq n$. For any lattice $\mathbb{A}$, we let $\mathbb{A}^{1}:=\mathbb{A}$ and $\mathbb{A}^{\partial}$ be the dual lattice, i.e. the lattice associated with the converse partial order of $\mathbb{A}$. For any order type $\varepsilon$, we let $\mathbb{A}^{\varepsilon}:=\Pi_{i=1}^{n} \mathbb{A}^{\varepsilon_{i}}$.

The language $\mathcal{L}_{\mathrm{DLE}}\left(\mathcal{F}, \mathcal{G}\right.$ ) (from now on abbreviated as $\mathcal{L}_{\mathrm{DLE}}$ ) takes as parameters: (i) a denumerable set of proposition letters AtProp, elements of which are denoted $p, q, r$, possibly with indexes; (ii) disjoint sets of connectives $\mathcal{F}$ and $\mathcal{G} .{ }^{11}$ Each $f \in \mathcal{F}$ and $g \in \mathcal{G}$ has arity $n_{f} \in \mathbb{N}$ (resp. $n_{g} \in \mathbb{N}$ ) and is associated with some order type $\varepsilon_{f}$ over $n_{f}$ (resp. $\varepsilon_{g}$ over $n_{g}$ ). ${ }^{12}$ The terms (formulas) of $\mathcal{L}_{\text {DLE }}$ are defined recursively as follows:

$$
\phi::=p|\perp| \top|\phi \wedge \phi| \phi \vee \phi|f(\bar{\phi})| g(\bar{\phi})
$$

where $p \in$ AtProp, $f \in \mathcal{F}, g \in \mathcal{G}$. Terms in $\mathcal{L}_{\text {DLE }}$ will be denoted either by $s, t$, or by lowercase Greek letters such as $\varphi, \psi, \gamma$ etc. In the context of sequents and prooftrees, $\mathcal{L}_{\text {DLE}}$-formulas will be denoted by uppercase letters $A, B$, etc.

\section{DEFINITION 9}

For any tuple $(\mathcal{F}, \mathcal{G})$ of disjoint sets of function symbols as above, a distributive lattice expansion (abbreviated as DLE) is a tuple $\mathbb{A}=\left(D, \mathcal{F}^{\mathbb{A}}, \mathcal{G}^{\mathbb{A}}\right)$ such that $D$ is a bounded distributive lattice (BDL), $\mathcal{F}^{\mathbb{A}}=\left\{f^{\mathbb{A}} \mid f \in \mathcal{F}\right\}$ and $\mathcal{G}^{\mathbb{A}}=\left\{g^{\mathbb{A}} \mid g \in \mathcal{G}\right\}$, such that every $f^{\mathbb{A}} \in \mathcal{F}^{\mathbb{A}}$ (resp. $g^{\mathbb{A}} \in \mathcal{G}^{\mathbb{A}}$ ) is an $n_{f}$-ary (resp. $n_{g}$-ary) operation on $\mathbb{A}$. A DLE is normal if every $f^{\mathbb{A}} \in \mathcal{F}^{\mathbb{A}}$ (resp. $g^{\mathbb{A}} \in \mathcal{G}^{\mathbb{A}}$ ) preserves finite joins (resp. meets) in each coordinate with $\varepsilon_{f}(i)=1$ (resp. $\left.\varepsilon_{g}(i)=1\right)$ and reverses finite meets (resp. joins) in each coordinate with $\varepsilon_{f}(i)=\partial$ (resp. $\varepsilon_{g}(i)=\partial$ ). ${ }^{13}$ Let $\mathbb{D L} \mathbb{E}$ be the class of DLEs. Sometimes we will refer to certain DLEs as $\mathcal{L}_{\text {DLE}}$-algebras when we wish to emphasize that these algebras have a compatible signature with the logical language we have fixed.

In the remainder of the article, we will abuse notation and write e.g. $f$ for $f^{\mathbb{A}}$. Normal DLEs constitute the main semantic environment of the present article. Henceforth, every DLE is assumed to be normal; hence the adjective 'normal' will be typically dropped. The class of all DLEs is equational, and can be axiomatized by the usual distributive lattice identities and the following equations for any $f \in \mathcal{F}$ (resp. $g \in \mathcal{G})$ and $1 \leq i \leq n_{f}$ (resp. for each $1 \leq j \leq n_{g}$ ):

- if $\varepsilon_{f}(i)=1$, then $f\left(p_{1}, \ldots, p \vee q, \ldots, p_{n_{f}}\right)=f\left(p_{1}, \ldots, p, \ldots, p_{n_{f}}\right) \vee f\left(p_{1}, \ldots, q, \ldots, p_{n_{f}}\right) \quad$ and $f\left(p_{1}, \ldots, \perp, \ldots, p_{n_{f}}\right)=\perp$,

- if $\varepsilon_{f}(i)=\partial$, then $f\left(p_{1}, \ldots, p \wedge q, \ldots, p_{n_{f}}\right)=f\left(p_{1}, \ldots, p, \ldots, p_{n_{f}}\right) \vee f\left(p_{1}, \ldots, q, \ldots, p_{n_{f}}\right) \quad$ and $f\left(p_{1}, \ldots, \top, \ldots, p_{n_{f}}\right)=\perp$,

${ }^{10}$ Throughout the article, order types will be typically associated with arrays of variables $\vec{p}:=\left(p_{1}, \ldots, p_{n}\right)$. When the order of the variables in $\vec{p}$ is not specified, we will sometimes abuse notation and write $\varepsilon(p)=1$ or $\varepsilon(p)=\partial$.

${ }^{11}$ It will be clear from the treatment in the present and the following sections that the connectives in $\mathcal{F}$ (resp. $\mathcal{G}$ ) correspond to those referred to as positive (resp. negative) connectives in [7]. The reason why this terminology is not adopted in the present article is explained later on in Footnote 21. Our assumption that the sets $\mathcal{F}$ and $\mathcal{G}$ are disjoint is motivated by the desideratum of generality and modularity. Indeed, for instance, the order theoretic properties of Boolean negation $\neg$ guarantee that this connective belongs both to $\mathcal{F}$ and to $\mathcal{G}$. In such cases, we prefer to define two copies $\neg \mathcal{F} \in \mathcal{F}$ and $\neg \mathcal{G} \in \mathcal{G}$, and introduce structural rules which encode the fact that these two copies coincide.

${ }^{12}$ Unary $f$ (resp. $g$ ) will be sometimes denoted as $\diamond$ (resp. $\square$ ) if the order type is 1 , and $\triangleleft$ (resp. $\triangleright$ ) if the order type is $\partial$.

13 Normal DLEs are sometimes referred to as distributive lattices with operators. This terminology directly derives from the setting of Boolean algebras with operators, in which operators are understood as operations which preserve finite meets in each coordinate. However, this terminology results somewhat ambiguous in the lattice setting, in which primitive operations are typically maps which are operators if seen as $\mathbb{A}^{\varepsilon} \rightarrow \mathbb{A}^{\eta}$ for some order type $\varepsilon$ on $n$ and some order type $\eta \in\{1, \partial\}$. Rather than speaking of distributive lattices with $(\varepsilon, \eta)$-operators, we then speak of normal DLEs. 
- if $\varepsilon_{g}(j)=1$, then $g\left(p_{1}, \ldots, p \wedge q, \ldots, p_{n_{g}}\right)=g\left(p_{1}, \ldots, p, \ldots, p_{n_{g}}\right) \wedge g\left(p_{1}, \ldots, q, \ldots, p_{n_{g}}\right) \quad$ and $g\left(p_{1}, \ldots, \top, \ldots, p_{n_{g}}\right)=\top$,

- if $\varepsilon_{g}(j)=\partial$, then $g\left(p_{1}, \ldots, p \vee q, \ldots, p_{n_{g}}\right)=g\left(p_{1}, \ldots, p, \ldots, p_{n_{g}}\right) \wedge g\left(p_{1}, \ldots, q, \ldots, p_{n_{g}}\right) \quad$ and $g\left(p_{1}, \ldots, \perp, \ldots, p_{n_{g}}\right)=\mathrm{T}$.

Each language $\mathcal{L}_{\text {DLE }}$ is interpreted in the appropriate class of DLEs. In particular, for every DLE $\mathbb{A}$, each operation $f^{\mathbb{A}} \in \mathcal{F}^{\mathbb{A}}$ (resp. $g^{\mathbb{A}} \in \mathcal{G}^{\mathbb{A}}$ ) is finitely join-preserving (resp. meet-preserving) in each coordinate when regarded as a map $f^{\mathbb{A}}: \mathbb{A}^{\varepsilon_{f}} \rightarrow \mathbb{A}\left(\right.$ resp. $\left.g^{\mathbb{A}}: \mathbb{A}^{\varepsilon_{g}} \rightarrow \mathbb{A}\right)$.

The generic DLE-logic is not equivalent to a sentential logic. Hence, the consequence relation of these logics cannot be uniformly captured in terms of theorems, but rather in terms of sequents, which motivates the following definition:

\section{DEFINITION 10}

For any language $\mathcal{L}_{\mathrm{DLE}}=\mathcal{L}_{\mathrm{DLE}}(\mathcal{F}, \mathcal{G})$, the basic, or minimal $\mathcal{L}_{\mathrm{DLE}}$-logic is a set of sequents $\phi \vdash \psi$, with $\phi, \psi \in \mathcal{L}_{\text {DLE }}$, which contains the following axioms:

- Sequents for lattice operations ${ }^{14}$ :

$$
\begin{array}{llll}
p \vdash p, & \perp \vdash p, & p \vdash \top, & p \wedge(q \vee r) \vdash(p \wedge q) \vee(p \wedge r), \\
p \vdash p \vee q, & q \vdash p \vee q, & p \wedge q \vdash p, & p \wedge q \vdash q,
\end{array}
$$

- Sequents for additional connectives:

$$
\begin{aligned}
& f\left(p_{1}, \ldots, \perp, \ldots, p_{n_{f}}\right) \vdash \perp, \text { for } \varepsilon_{f}(i)=1, \\
& f\left(p_{1}, \ldots, \top, \ldots, p_{n_{f}}\right) \vdash \perp, \text { for } \varepsilon_{f}(i)=\partial, \\
& \top \vdash g\left(p_{1}, \ldots, \top, \ldots, p_{n_{g}}\right), \text { for } \varepsilon_{g}(i)=1, \\
& \top \vdash g\left(p_{1}, \ldots, \perp, \ldots, p_{n_{g}}\right), \text { for } \varepsilon_{g}(i)=\partial, \\
& f\left(p_{1}, \ldots, p \vee q, \ldots, p_{n_{f}}\right) \vdash f\left(p_{1}, \ldots, p, \ldots, p_{n_{f}}\right) \vee f\left(p_{1}, \ldots, q, \ldots, p_{n_{f}}\right), \text { for } \varepsilon_{f}(i)=1, \\
& f\left(p_{1}, \ldots, p \wedge q, \ldots, p_{n_{f}}\right) \vdash f\left(p_{1}, \ldots, p, \ldots, p_{n_{f}}\right) \vee f\left(p_{1}, \ldots, q, \ldots, p_{n_{f}}\right), \text { for } \varepsilon_{f}(i)=\partial, \\
& g\left(p_{1}, \ldots, p, \ldots, p_{n_{g}}\right) \wedge g\left(p_{1}, \ldots, q, \ldots, p_{n_{g}}\right) \vdash g\left(p_{1}, \ldots, p \wedge q, \ldots, p_{n_{g}}\right), \text { for } \varepsilon_{g}(i)=1, \\
& g\left(p_{1}, \ldots, p, \ldots, p_{n_{g}}\right) \wedge g\left(p_{1}, \ldots, q, \ldots, p_{n_{g}}\right) \vdash g\left(p_{1}, \ldots, p \vee q, \ldots, p_{n_{g}}\right), \text { for } \varepsilon_{g}(i)=\partial,
\end{aligned}
$$

and is closed under the following inference rules:

$$
\begin{gathered}
\frac{\phi \vdash \chi \quad \chi \vdash \psi}{\phi \vdash \psi} \frac{\phi \vdash \psi}{\phi(\chi / p) \vdash \psi(\chi / p)} \frac{\chi \vdash \phi \quad \chi \vdash \psi}{\chi \vdash \phi \wedge \psi} \quad \frac{\phi \vdash \chi \quad \psi \vdash \chi}{\phi \vee \psi \vdash \chi} \\
\frac{\phi \vdash \psi}{f\left(p_{1}, \ldots, \phi, \ldots, p_{n}\right) \vdash f\left(p_{1}, \ldots, \psi, \ldots, p_{n}\right)}\left(\varepsilon_{f}(i)=1\right) \\
\frac{\phi \vdash \psi}{f\left(p_{1}, \ldots, \psi, \ldots, p_{n}\right) \vdash f\left(p_{1}, \ldots, \phi, \ldots, p_{n}\right)}\left(\varepsilon_{f}(i)=\partial\right) \\
\frac{\phi \vdash \psi}{g\left(p_{1}, \ldots, \phi, \ldots, p_{n}\right) \vdash g\left(p_{1}, \ldots, \psi, \ldots, p_{n}\right)}\left(\varepsilon_{g}(i)=1\right)
\end{gathered}
$$

\footnotetext{
${ }^{14}$ In what follows we will use the turnstile symbol $\vdash$ both as sequent separator and also as the consequence relation of the logic.
} 


$$
\frac{\phi \vdash \psi}{g\left(p_{1}, \ldots, \psi, \ldots, p_{n}\right) \vdash g\left(p_{1}, \ldots, \phi, \ldots, p_{n}\right)}\left(\varepsilon_{g}(i)=\partial\right) .
$$

The minimal DLE-logic is denoted by $\mathbf{L}_{\text {DLE }}$. For any DLE-language $\mathcal{L}_{\text {DLE }}$, by a DLE-logic we understand any axiomatic extension of the basic $\mathcal{L}_{\text {DLE-logic in }} \mathcal{L}_{\text {DLE }}$.

For every DLE $\mathbb{A}$, the symbol $\vdash$ is interpreted as the lattice order $\leq$. A sequent $\phi \vdash \psi$ is valid in $\mathbb{A}$ if $h(\phi) \leq h(\psi)$ for every homomorphism $h$ from the $\mathcal{L}_{\text {DLE-algebra of formulas over AtProp }}$ to $\mathbb{A}$. The notation $\mathbb{D} \mathbb{E} \models \phi \vdash \psi$ indicates that $\phi \vdash \psi$ is valid in every DLE. Then, by means of a routine Lindenbaum-Tarski construction, it can be shown that the minimal DLE-logic $\mathbf{L}_{\text {DLE }}$ is sound and complete with respect to its correspondent class of algebras $\mathbb{D L E} \mathbb{E}$, i.e. that any sequent $\phi \vdash \psi$ is provable in $\mathbf{L}_{\text {DLE }}$ iff $\mathbb{D L} \mathbb{E} \models \phi \vdash \psi$.

\subsection{The expanded language $\mathcal{L}_{\mathrm{DLE}}^{*}$}

Any given language $\mathcal{L}_{\text {DLE }}=\mathcal{L}_{\text {DLE }}(\mathcal{F}, \mathcal{G})$ can be associated with the language $\mathcal{L}_{\text {DLE }}^{*}=\mathcal{L}_{\text {DLE }}\left(\mathcal{F}^{*}, \mathcal{G}^{*}\right)$, where $\mathcal{F}^{*} \supseteq \mathcal{F}$ and $\mathcal{G}^{*} \supseteq \mathcal{G}$ are obtained by expanding $\mathcal{L}_{\text {DLE }}$ with the following connectives:

(1) the binary connectives $\leftarrow$ and $\rightarrow$, the intended interpretations of which are the right residuals of $\wedge$ in the first and second coordinate, respectively, and $\prec$ and $\succ$, the intended interpretations of which are the left residuals of $\vee$ in the first and second coordinate, respectively;

(2) the $n_{f}$-ary connective $f_{i}^{\sharp}$ for $0 \leq i \leq n_{f}$, the intended interpretation of which is the right residual of $f \in \mathcal{F}$ in its $i$-th coordinate if $\varepsilon_{f}(i)=1$ (resp. its Galois-adjoint if $\varepsilon_{f}(i)=\partial$ );

(3) the $n_{g}$-ary connective $g_{i}^{b}$ for $0 \leq i \leq n_{g}$, the intended interpretation of which is the left residual of $g \in \mathcal{G}$ in its $i$-th coordinate if $\varepsilon_{g}(i)=1$ (resp. its Galois-adjoint if $\varepsilon_{g}(i)=\partial$ ). ${ }^{15}$

We stipulate that $>, \prec \in \mathcal{F}^{*}$, that $\rightarrow, \leftarrow \in \mathcal{G}^{*}$, and moreover, that $f_{i}^{\sharp} \in \mathcal{G}^{*}$ if $\varepsilon_{f}(i)=1$, and $f_{i}^{\sharp} \in \mathcal{F}^{*}$ if $\varepsilon_{f}(i)=\partial$. Dually, $g_{i}^{b} \in \mathcal{F}^{*}$ if $\varepsilon_{g}(i)=1$, and $g_{i}^{b} \in \mathcal{G}^{*}$ if $\varepsilon_{g}(i)=\partial$. The order type assigned to the additional connectives is predicated on the order type of their intended interpretations. That is, for any $f \in \mathcal{F}$ and $g \in \mathcal{G}$,

(1) if $\varepsilon_{f}(i)=1$, then $\varepsilon_{f_{i}^{\sharp}}(i)=1$ and $\varepsilon_{f_{i}^{\sharp}}(j)=\left(\varepsilon_{f}(j)\right)^{\partial}$ for any $j \neq i$.

(2) if $\varepsilon_{f}(i)=\partial$, then $\varepsilon_{f_{i}^{\sharp}}(i)=\partial$ and $\varepsilon_{f_{i}^{\sharp}}(j)=\varepsilon_{f}(j)$ for any $j \neq i$.

(3) if $\varepsilon_{g}(i)=1$, then $\varepsilon_{g_{i}}(i)=1$ and $\varepsilon_{g_{i}}(j)=\left(\varepsilon_{g}(j)\right)^{\partial}$ for any $j \neq i$.

(4) if $\varepsilon_{g}(i)=\partial$, then $\varepsilon_{g_{i}}(i)=\partial$ and $\varepsilon_{g_{i}}(j)=\varepsilon_{g}(j)$ for any $j \neq i$.

For instance, if $f$ and $g$ are binary connectives such that $\varepsilon_{f}=(1, \partial)$ and $\varepsilon_{g}=(\partial, 1)$, then $\varepsilon_{f_{1}^{\sharp}}=(1,1)$, $\varepsilon_{f_{2}^{\sharp}}=(1, \partial), \varepsilon_{g_{1}^{b}}=(\partial, 1)$ and $\varepsilon_{g_{2}^{b}}=(1,1){ }^{16}$

DEFINITION 11

For any language $\mathcal{L}_{\mathrm{DLE}}(\mathcal{F}, \mathcal{G})$, the basic bi-intuitionistic 'tense' $\mathcal{L}_{\mathrm{DLE}}$-logic is defined by specializing Definition 10 to the language $\mathcal{L}_{\text {DLE }}^{*}=\mathcal{L}_{\text {DLE }}\left(\mathcal{F}^{*}, \mathcal{G}^{*}\right)$ and closing under the following additional rules:

\footnotetext{
${ }^{15}$ The adjoints of the unary connectives $\square, \diamond, \triangleleft$ and $\triangleright$ are denoted $\diamond, \mathbf{\square}, \triangleleft$ and $\downarrow$, respectively.

${ }^{16}$ Warning: notice that this notation heavily depends from the connective which is taken as primitive, and needs to be carefully adapted to well known cases. For instance, consider the 'fusion' connective $\circ$ (which, when denoted as $f$, is such that $\left.\varepsilon_{f}=(1,1)\right)$. Its residuals $f_{1}^{\sharp}$ and $f_{2}^{\sharp}$ are commonly denoted / and $\backslash$, respectively. However, if $\backslash$ is taken as the primitive connective $g$, then $g_{2}^{b}$ is $\circ=f$, and $g_{1}^{b}\left(x_{1}, x_{2}\right):=x_{2} / x_{1}=f_{1}^{\sharp}\left(x_{2}, x_{1}\right)$. This example shows that, when identifying $g_{1}^{b}$ and $f_{1}^{\sharp}$, the conventional order of the coordinates is not preserved, and depends of which connective is taken as primitive.
} 
(1) Residuation rules for lattice connectives:

$$
\frac{\phi \wedge \psi \vdash \chi}{\overline{\psi \vdash \phi \rightarrow \chi}} \frac{\phi \wedge \psi \vdash \chi}{\overline{\phi \vdash \chi \leftarrow \psi}} \frac{\phi \vdash \psi \vee \chi}{\psi>\phi \vdash \chi} \frac{\phi \vdash \psi \vee \chi}{\overline{\phi-\chi \vdash \psi}}
$$

Notice that the rules for $\rightarrow$ and $\leftarrow$ are interderivable, since $\wedge$ is commutative; similarly, the rules for $>$ and $<$ are interderivable, since $\vee$ is commutative.

(2) Residuation rules for $f \in \mathcal{F}$ and $g \in \mathcal{G}$ :

$$
\begin{aligned}
& \left(\varepsilon_{f}(i)=1\right) \frac{f\left(\varphi_{1}, \ldots, \phi, \ldots, \varphi_{n_{f}}\right) \vdash \psi}{\phi \vdash f_{i}^{\sharp}\left(\varphi_{1}, \ldots, \psi, \ldots, \varphi_{n_{f}}\right)} \frac{\phi \vdash g\left(\varphi_{1}, \ldots, \psi, \ldots, \varphi_{n_{g}}\right)}{g_{i}^{\mathrm{b}}\left(\varphi_{1}, \ldots, \phi, \ldots, \varphi_{n_{g}}\right) \vdash \psi}\left(\varepsilon_{g}(i)=1\right) \\
& \left(\varepsilon_{f}(i)=\partial\right) \frac{f\left(\varphi_{1}, \ldots, \phi, \ldots, \varphi_{n_{f}}\right) \vdash \psi}{\frac{\phi \vdash g\left(\varphi_{1}, \ldots, \psi, \ldots, \varphi_{n_{g}}\right)}{f_{i}^{\sharp}\left(\varphi_{1}, \ldots, \psi, \ldots, \varphi_{n_{f}}\right) \vdash \phi}} \frac{\phi \vdash g_{i}^{\mathrm{b}}\left(\varphi_{1}, \ldots, \phi, \ldots, \varphi_{n_{g}}\right)}{\psi}\left(\varepsilon_{g}(i)=\partial\right)
\end{aligned}
$$

The double line in each rule above indicates that the rule is invertible. Let $\mathbf{L}_{\text {DLE }}^{*}$ be the minimal biintuitionistic 'tense' $\mathcal{L}_{\text {DLE-logic. }}{ }^{17}$ For any DLE-language $\mathcal{L}_{\text {DLE }}$, by a tense DLE-logic we understand any axiomatic extension of the basic tense bi-intuitionistic $\mathcal{L}_{\text {DLE }}$-logic in $\mathcal{L}_{\text {DLE }}^{*}$.

The algebraic semantics of $\mathbf{L}_{\text {DLE }}^{*}$ is given by the class of bi-intuitionistic 'tense' $\mathcal{L}_{\text {DLE}}$-algebras, defined as tuples $\mathbb{A}=\left(H, \mathcal{F}^{*}, \mathcal{G}^{*}\right)$ such that $H$ is a bi-Heyting algebra, ${ }^{18}$ and moreover:

(1) For every $f \in \mathcal{F}$ s.t. $n_{f} \geq 1$, all $a_{1}, \ldots, a_{n_{f}} \in D$ and $b \in D$, and each $1 \leq i \leq n_{f}$,

- if $\varepsilon_{f}(i)=1$, then $f\left(a_{1}, \ldots, a_{i}, \ldots a_{n_{f}}\right) \leq b$ iff $a_{i} \leq f_{i}^{\sharp}\left(a_{1}, \ldots, b, \ldots, a_{n_{f}}\right)$;

- if $\varepsilon_{f}(i)=\partial$, then $f\left(a_{1}, \ldots, a_{i}, \ldots a_{n_{f}}\right) \leq b$ iff $a_{i} \leq^{\partial} f_{i}^{\sharp}\left(a_{1}, \ldots, b, \ldots, a_{n_{f}}\right)$.

(2) For every $g \in \mathcal{G}$ s.t. $n_{g} \geq 1$, any $a_{1}, \ldots, a_{n_{g}} \in D$ and $b \in D$, and each $1 \leq i \leq n_{g}$,

- if $\varepsilon_{g}(i)=1$, then $b \leq g\left(a_{1}, \ldots, a_{i}, \ldots a_{n_{g}}\right)$ iff $g_{i}^{b}\left(a_{1}, \ldots, b, \ldots, a_{n_{g}}\right) \leq a_{i}$.

- if $\varepsilon_{g}(i)=\partial$, then $b \leq g\left(a_{1}, \ldots, a_{i}, \ldots a_{n_{g}}\right)$ iff $g_{i}^{\mathrm{b}}\left(a_{1}, \ldots, b, \ldots, a_{n_{g}}\right) \leq{ }^{\partial} a_{i}$.

It is also routine to prove using the Lindenbaum-Tarski construction that $\mathbf{L}_{\mathrm{DLE}}^{*}$ (as well as any of its sound axiomatic extensions) is sound and complete w.r.t. the class of bi-intuitionistic 'tense'

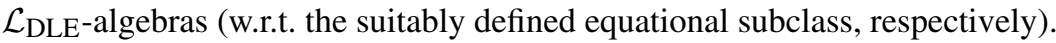

THEOREM 12

The logic $\mathbf{L}_{\mathrm{DLE}}^{*}$ is a conservative extension of $\mathbf{L}_{\mathrm{DLE}}$, i.e. for every $\mathcal{L}_{\mathrm{DLE}}$-sequent $\phi \vdash \psi, \phi \vdash \psi$ is derivable in $\mathbf{L}_{\text {DLE }}$ iff $\phi \vdash \psi$ is derivable in $\mathbf{L}_{\text {DLE }}^{*}$. Moreover, every DLE-logic can be extended conservatively to a DLE*-logic.

${ }^{17}$ Hence, for any language $\mathcal{L}_{\mathrm{DLE}}$, there are in principle two logics associated with the expanded language $\mathcal{L}_{\mathrm{DLE}}^{*}$, namely the minimal $\mathcal{L}_{\mathrm{DLE}}^{*}$-logic, which we denote by $\mathbf{L}_{\mathrm{DLE}}^{*}$, and which is obtained by instantiating Definition 10 to the language $\mathcal{L}_{\mathrm{DLE}}^{*}$, and the bi-intuitionistic 'tense' logic $\mathbf{L}_{\mathrm{DLE}}^{*}$, defined above. The logic $\mathbf{L}_{\mathrm{DLE}}^{*}$ is the natural logic on the language $\mathcal{L}_{\mathrm{DLE}}^{*}$, however, it is useful to introduce a specific notation for $\mathbf{L}_{\mathrm{DLE}}^{*}$, given that all the results holding for the minimal logic associated with an arbitrary DLE-language can be instantiated to the expanded language $\mathcal{L}_{\text {DLE }}^{*}$ and will then apply to $\mathbf{L}_{\mathrm{DLE}}^{*}$.

${ }^{18}$ That is, $H=(D, \rightarrow,-<)$ such that both $(D, \rightarrow)$ and $\left(D^{\partial},-<\right)$ are Heyting algebras. In particular, setting $c \leftarrow b:=b \rightarrow c$ and $b>-a:=a-b$ for all $a, b, c \in D$, the following equivalences hold

$$
a \wedge b \leq c \text { iff } b \leq a \rightarrow c \text { iff } a \leq c \leftarrow b, \quad a \leq b \vee c \text { iff } b>a \leq c \text { iff } a<c \leq b .
$$


ProOF. We only outline the proof. Clearly, every $\mathcal{L}_{\mathrm{DLE}}$-sequent which is $\mathbf{L}_{\mathrm{DLE}}$-derivable is also $\mathbf{L}_{\mathrm{DLE}}^{*}$-derivable. Conversely, if an $\mathcal{L}_{\mathrm{DLE}}$-sequent $\phi \vdash \psi$ is not $\mathbf{L}_{\mathrm{DLE}}$-derivable, then by the

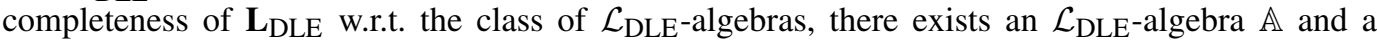
variable assignment $v$ under which $\phi^{\mathbb{A}} \not \psi^{\mathbb{A}}$. Consider the canonical extension $\mathbb{A}^{\delta}$ of $\mathbb{A} .{ }^{19}$ Since $\mathbb{A}$ is a subalgebra of $\mathbb{A}^{\delta}$, the sequent $\phi \vdash \psi$ is not satisfied in $\mathbb{A}^{\delta}$ under the variable assignment $\iota$ $\nu(\iota$ denoting the canonical embedding $\mathbb{A} \hookrightarrow \mathbb{A}^{\delta}$ ). Moreover, since $\mathbb{A}^{\delta}$ is a perfect $\mathcal{L}_{\text {DLE }}$-algebra, it is naturally endowed with a structure of bi-intuitionistic 'tense' $\mathcal{L}_{\mathrm{DLE}}$-algebra. Thus, by the completeness of $\mathbf{L}_{\text {DLE }}^{*}$ w.r.t. the class of bi-intuitionistic 'tense' $\mathcal{L}_{\text {DLE }}$-algebras, the sequent $\phi \vdash \psi$ is not derivable in $\mathbf{L}_{\mathrm{DLE}}^{*}$, as required.

Notice that the algebraic completeness of the logics $\mathbf{L}_{\mathrm{DLE}}$ and $\mathbf{L}_{\mathrm{DLE}}^{*}$ and the canonical embedding of DLEs into their canonical extensions immediately give completeness of $\mathbf{L}_{\mathrm{DLE}}$ and $\mathbf{L}_{\text {DLE }}^{*}$ w.r.t. the appropriate class of perfect DLEs.

\subsection{The algorithm ALBA, informally}

The contribution of the present article is an application of unified correspondence theory [16, 18], of which the algorithm ALBA is one of the main tools. In the present subsection, we will guide the reader through the main principles which make it work, by means of an example. This presentation is based on analogous illustrations in [14] and [22].

Let us start with one of the best known examples in correspondence theory, namely $\diamond \square p \rightarrow \square \diamond p$. It is well known that for every Kripke frame $\mathbb{F}=(W, R)$,

$$
\mathbb{F} \Vdash \diamond \square p \rightarrow \square \diamond p \text { iff } \mathbb{F} \models \forall x y z(R x y \wedge R x z \rightarrow \exists u(R y u \wedge R z u)) .
$$

As is discussed at length in $[16,18]$, every piece of argument used to prove this correspondence on frames can be translated by duality to complex algebras (cf. [5, Definition 5.21]). We will show how this is done in the case of the example above.

As is well known, complex algebras are characterized in purely algebraic terms as complete and atomic Boolean Algebras with Operators (BAOs) where the modal operations are completely join-preserving. These are also known as perfect BAOs [6, Definition 40, Chapter 6].

First of all, the condition $\mathbb{F} \Vdash \diamond \square p \rightarrow \square \diamond p$ translates to the complex algebra $\mathbb{A}=\mathbb{F}^{+}$of $\mathbb{F}$ as $\llbracket \diamond \square p \rrbracket \subseteq \llbracket \square \diamond p \rrbracket$ for every assignment of $p$ into $A$, so this validity clause can be rephrased as follows:

$$
\mathbb{A} \models \forall p[\diamond \square p \leq \square \diamond p],
$$

where the order $\leq$ is interpreted as set inclusion in the complex algebra. In perfect BAOs, every element is both the join of the completely join-prime elements (the set of which is denoted $J^{\infty}(\mathbb{A})$ )

19 The canonical extension of a BDL $D$ is a complete distributive lattice $D^{\delta}$ containing $D$ as a sublattice, such that:

(1) (denseness) every element of $D^{\delta}$ can be expressed both as a join of meets and as a meet of joins of elements from $D$;

(2) (compactness) for all $S, T \subseteq D$, if $\bigwedge S \leq \bigvee T$ in $D^{\delta}$, then $\bigwedge F \leq \bigvee G$ for some finite sets $F \subseteq S$ and $G \subseteq T$.

It is well known that the canonical extension of a BDL $D$ is unique up to isomorphism fixing $D$ (cf. e.g. [33, Section 2.2]), and that the canonical extension of a BDL is a perfect BDL, i.e. a complete and completely distributive lattice which is completely join-generated by its completely join-irreducible elements and completely meet-generated by its completely meet-irreducible elements (cf. e.g. [33, Definition 2.14]). The canonical extension of an $\mathcal{L}_{\text {DLE}}$-algebra $\mathbb{A}=\left(D, \mathcal{F}^{\mathbb{A}}, \mathcal{G}^{\mathbb{A}}\right)$ is the

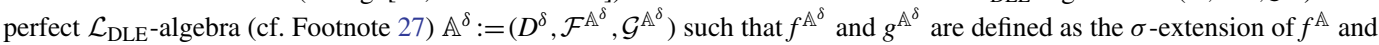
as the $\pi$-extension of $g^{\mathbb{A}}$, respectively, for all $f \in \mathcal{F}$ and $g \in \mathcal{G}$ (cf. [54, 55]). 
below it and the meet of the completely meet-prime elements (the set of which is denoted $M^{\infty}(\mathbb{A})$ ) above it. ${ }^{20}$ Hence, taking some liberties in our use of notation, the condition above can be equivalently rewritten as follows:

$$
\mathbb{A} \models \forall p\left[\bigvee\left\{i \in J^{\infty}(\mathbb{A}) \mid i \leq \square \diamond p\right\} \leq \bigwedge\left\{m \in M^{\infty}(\mathbb{A}) \mid \square \diamond p \leq m\right\}\right]
$$

By elementary properties of least upper bounds and greatest lower bounds in posets (cf. [26]), this condition is true if and only if every element in the join is less than or equal to every element in the meet; thus, condition (3.1) above can be rewritten as:

$$
\mathbb{A} \models \forall p \forall \mathbf{i} \forall \mathbf{m}[(\mathbf{i} \leq \diamond \square p \& \square \diamond p \leq \mathbf{m}) \Rightarrow \mathbf{i} \leq \mathbf{m}],
$$

where the variables $\mathbf{i}$ and $\mathbf{m}$ range over $J^{\infty}(\mathbb{A})$ and $M^{\infty}(\mathbb{A})$, respectively (following the literature, we will refer to the former variables as nominals, and to the latter ones as co-nominals). Since $\mathbb{A}$ is a perfect $\mathrm{BAO}$, the element of $\mathbb{A}$ interpreting $\square p$ is the join of the completely join-prime elements below it. Hence, if $i \in J^{\infty}(\mathbb{A})$ and $i \leq \diamond \square p$, because $\diamond$ is completely join-preserving on $\mathbb{A}$, we have that

$$
i \leq \diamond\left(\bigvee\left\{j \in J^{\infty}(\mathbb{A}) \mid j \leq \square p\right\}\right)=\bigvee\left\{\diamond j \mid j \in J^{\infty}(\mathbb{A}) \text { and } j \leq \square p\right\},
$$

which implies that $i \leq \diamond_{0}$ for some $j_{0} \in J^{\infty}(\mathbb{A})$ such that $j_{0} \leq \square p$. Hence, we can equivalently rewrite the validity clause above as follows:

$$
\mathbb{A} \models \forall p \forall \mathbf{i} \forall \mathbf{m}[(\exists \mathbf{j}(\mathbf{i} \leq \diamond \mathbf{j} \& \mathbf{j} \leq \square p) \& \square \diamond p \leq \mathbf{m}) \Rightarrow \mathbf{i} \leq \mathbf{m}],
$$

and then use standard manipulations from first-order logic to pull out quantifiers:

$$
\mathbb{A} \models \forall p \forall \mathbf{i} \forall \mathbf{m} \forall \mathbf{j}[(\mathbf{i} \leq \diamond \mathbf{j} \& \mathbf{j} \leq \square p \& \square \diamond p \leq \mathbf{m}) \Rightarrow \mathbf{i} \leq \mathbf{m}] .
$$

Now we observe that the operation $\square$ preserves arbitrary meets in the perfect BAO $\mathbb{A}$. By the general theory of adjunction in complete lattices, this is equivalent to $\square$ being a right adjoint (cf. [26, Proposition 7.34]). It is also well known that the left or lower adjoint (cf. [26, Definition 7.23]) of $\square$ is the operation $\downarrow$, which can be recognized as the backward-looking diamond $P$, interpreted with the converse $R^{-1}$ of the accessibility relation $R$ of the frame $\mathbb{F}$ in the context of tense logic (cf. [5, Example 1.25] and [26, Exercise 7.18] modulo translating the notation). Hence, the condition above can be equivalently rewritten as:

$$
\mathbb{A} \models \forall p \forall \mathbf{i} \forall \mathbf{m} \forall \mathbf{j}[(\mathbf{i} \leq \diamond \mathbf{j} \& \diamond \mathbf{j} \leq p \& \square \diamond p \leq \mathbf{m}) \Rightarrow \mathbf{i} \leq \mathbf{m}],
$$

and then as follows:

$$
\mathbb{A} \models \forall \mathbf{i} \forall \mathbf{m} \forall \mathbf{j}[(\mathbf{i} \leq \diamond \mathbf{j} \& \exists p(\diamond \mathbf{j} \leq p \& \square \diamond p \leq \mathbf{m})) \Rightarrow \mathbf{i} \leq \mathbf{m}]
$$

At this point we are in a position to eliminate the variable $p$ and equivalently rewrite the previous condition as follows:

$$
\mathbb{A} \models \forall \mathbf{i} \forall \mathbf{m} \forall \mathbf{j}[(\mathbf{i} \leq \diamond \mathbf{j} \& \square \diamond \diamond \mathbf{j} \leq \mathbf{m}) \Rightarrow \mathbf{i} \leq \mathbf{m}] .
$$

Let us justify this equivalence: for the direction from top to bottom, fix an interpretation $V$ of the variables $\mathbf{i}, \mathbf{j}$ and $\mathbf{m}$ such that $\mathbf{i} \leq \diamond \mathbf{j}$ and $\square \diamond \bullet \mathbf{j} \leq \mathbf{m}$. To prove that $\mathbf{i} \leq \mathbf{m}$ holds under $V$, consider the

\footnotetext{
${ }^{20}$ In BAOs the completely join-prime elements, the completely join-irreducible elements and the atoms coincide. Moreover, the completely meet-prime elements, the completely meet-irreducible elements and the co-atoms coincide.
} 
variant $V^{*}$ of $V$ such that $V^{*}(p)=\bullet \mathbf{j}$. Then it can be easily verified that $V^{*}$ witnesses the antecedent of (3.6) under $V$; hence $\mathbf{i} \leq \mathbf{m}$ holds under $V$. Conversely, fix an interpretation $V$ of the variables $\mathbf{i}$, $\mathbf{j}$ and $\mathbf{m}$ such that $\mathbf{i} \leq \diamond \mathbf{j} \& \exists p(\diamond \mathbf{j} \leq p \& \square \diamond p \leq \mathbf{m})$. Then, by monotonicity, the antecedent of (3.7) holds under $V$, and hence so does $\mathbf{i} \leq \mathbf{m}$, as required. This is an instance of the following result, known as Ackermann's lemma ([1], see also [17]):

LEMMA 13

Fix an arbitrary propositional language $L$. Let $\alpha, \beta(p), \gamma(p)$ be $L$-formulas such that $\alpha$ is $p$-free, $\beta$ is positive and $\gamma$ is negative in $p$. For any assignment $V$ on an $L$-algebra $\mathbb{A}$, the following are equivalent:

(1) $\mathbb{A}, V=\beta(\alpha / p) \leq \gamma(\alpha / p)$;

(2) there exists a $p$-variant $V^{*}$ of $V$ such that $\mathbb{A}, V^{*} \models \alpha \leq p$ and $\mathbb{A}, V^{*} \models \beta(p) \leq \gamma(p)$,

where $\beta(\alpha / p)$ and $\gamma(\alpha / p)$ denote the result of uniformly substituting $\alpha$ for $p$ in $\beta$ and $\gamma$, respectively.

The proof is essentially the same as [18, Lemma 4.2]. Whenever, in a reduction, we reach a shape in which the lemma above (or its order-dual) can be applied, we say that the condition is in Ackermann shape.

Taking stock, we note that we have equivalently transformed (3.1) into (3.7), which is a condition in which all propositional variables (corresponding to monadic second-order variables) have been eliminated, and all remaining variables range over completely join- and meet-irreducible elements of the complex algebra $\mathbb{A}$. Via discrete Stone duality, these elements, respectively, correspond to singletons and complements of singletons of the Kripke frame from which $\mathbb{A}$ arises. Moreover, $\bullet$ is interpreted on Kripke frames using the converse of the same accessibility relation used to interpret $\square$. Hence, clause (3.7) translates equivalently into a condition in the first-order correspondence language of $\mathbb{F}$.

To facilitate this translation, we first rewrite (3.7) as follows, by reversing the reasoning that brought us from (3.1) to (3.2):

$$
\mathbb{A}=\forall \mathbf{j}[\diamond \mathbf{j} \leq \square \diamond \bullet \mathbf{j}]
$$

By again applying the fact that $\square$ is a right adjoint we obtain

$$
\mathbb{A} \models \forall \mathbf{j}[\diamond \diamond \mathbf{j} \leq \diamond \diamond \mathbf{j}]
$$

Recalling that $\mathbb{A}$ is the complex algebra of $\mathbb{F}=(W, R)$, we can interpret the variable $\mathbf{j}$ as an individual variable ranging in the universe $W$ of $\mathbb{F}$, and the operations $\diamond$ and $\bullet$ as the set-theoretic operations defined on $\mathcal{P}(W)$ by the assignments $X \mapsto R^{-1}[X]$ and $X \mapsto R[X]$, respectively. Hence, clause (3.9) above can be equivalently rewritten on the side of the frames as

$$
\mathbb{F} \models \forall w\left(R\left[R^{-1}[w]\right] \subseteq R^{-1}[R[w]]\right) .
$$

Notice that $R\left[R^{-1}[w]\right]$ is the set of all states $x \in W$ which have a predecessor $z$ in common with $w$, while $R^{-1}[R[w]]$ is the set of all states $x \in W$ which have a successor in common with $w$. This can be spelled out as

$$
\forall x \forall w(\exists z(R z x \wedge R z w) \rightarrow \exists y(R x y \wedge R w y))
$$

or, equivalently,

$$
\forall z \forall x \forall w((R z x \wedge R z w) \rightarrow \exists y(R x y \wedge R w y))
$$

which is the familiar Church-Rosser condition. 
Finally, the example above illustrates another important feature of the ALBA-based approach to the computation of first-order correspondents. Namely, ALBA-computations are neatly divided into two stages: the reduction stage, carried out from (3.1) into (3.7) in the example above; and the translation stage, in which the expressions (equalities and quasi-inequalities) obtained by eliminating all proposition variables from an input inequality are suitably translated into frame-correspondent language. Only the reduction stage will be relevant to the remainder of the present article.

\subsection{Inductive inequalities}

In the present subsection, we will report on the definition of inductive $\mathcal{L}_{\mathrm{DLE}}$-inequalities on which the algorithm ALBA is guaranteed to succeed (cf. [16, 18]).

\section{DEFINITION 14 (Signed Generation Tree)}

The positive (resp. negative) generation tree of any $\mathcal{L}_{\mathrm{DLE}}$-term $s$ is defined by labelling the root node of the generation tree of $s$ with the sign + (resp. - ), and then propagating the labelling on each remaining node as follows:

- For any node labelled with $\vee$ or $\wedge$, assign the same sign to its children nodes.

- For any node labelled with $h \in \mathcal{F} \cup \mathcal{G}$ of arity $n_{h} \geq 1$, and for any $1 \leq i \leq n_{h}$, assign the same (resp. the opposite) sign to its $i$-th child node if $\varepsilon_{h}(i)=1$ (resp. if $\varepsilon_{h}(i)=\partial$ ).

Nodes in signed generation trees are positive (resp. negative) if are signed $+($ resp. -$){ }^{21}$

Signed generation trees will be mostly used in the context of term inequalities $s \leq t$. In this context, we will typically consider the positive generation tree $+s$ for the left-hand side and the negative one $-t$ for the right-hand side. We will also say that a term-inequality $s \leq t$ is uniform in a given variable $p$ if all occurrences of $p$ in both $+s$ and $-t$ have the same sign, and that $s \leq t$ is $\varepsilon$-uniform in a (sub)array $\vec{p}$ of its variables if $s \leq t$ is uniform in $p$, occurring with the sign indicated by $\varepsilon$, for every $p$ in $\vec{p} .^{22}$

For any term $s\left(p_{1}, \ldots p_{n}\right)$, any order type $\varepsilon$ over $n$, and any $1 \leq i \leq n$, an $\varepsilon$-critical node in a signed generation tree of $s$ is a leaf node $+p_{i}$ with $\varepsilon_{i}=1$ or $-p_{i}$ with $\varepsilon_{i}=\partial$. An $\varepsilon$-critical branch in the tree is a branch from an $\varepsilon$-critical node. The intuition, which will be built upon later, is that variable occurrences corresponding to $\varepsilon$-critical nodes are to be solved for, according to $\varepsilon$.

For every term $s\left(p_{1}, \ldots p_{n}\right)$ and every order type $\varepsilon$, we say that $+s$ (resp. $\left.-s\right)$ agrees with $\varepsilon$, and write $\varepsilon(+s)$ (resp. $\varepsilon(-s)$ ), if every leaf in the signed generation tree of $+s$ (resp. $-s$ ) is $\varepsilon$-critical. In other words, $\varepsilon(+s)$ (resp. $\varepsilon(-s)$ ) means that all variable occurrences corresponding to leaves of $+s$ (resp. $-s$ ) are to be solved for according to $\varepsilon$. We will also write $+s^{\prime} \prec * s$ (resp. $-s^{\prime} \prec * s$ ) to indicate that the subterm $s^{\prime}$ inherits the positive (resp. negative) sign from the signed generation tree $* s$. Finally, we will write $\varepsilon(\gamma) \prec * s$ (resp. $\varepsilon^{\partial}(\gamma) \prec * s$ ) to indicate that the signed subtree $\gamma$, with the sign inherited from $* s$, agrees with $\varepsilon$ (resp. with $\varepsilon^{\partial}$ ).

\section{DEFINITION 15}

Nodes in signed generation trees will be called $\Delta$-adjoints, syntactically left residual (SLR), syntactically right residual (SRR) and syntactically right adjoint (SRA), according to the specification

\footnotetext{
21 The terminology used in [7] regarding 'positive' and 'negative connectives' has not been adopted in the present article to avoid confusion with positive and negative nodes in signed generation trees.

${ }^{22}$ The following observation will be used at various points in the remainder of the present article: if a term inequality $s(\vec{p}, \vec{q}) \leq t(\vec{p}, \vec{q})$ is $\varepsilon$-uniform in $\vec{p}$ (cf. discussion after Definition 14), then the validity of $s \leq t$ is equivalent to the validity of $s\left(\overrightarrow{T^{\varepsilon(i)}}, \vec{q}\right) \leq t\left(\overrightarrow{T^{\varepsilon(i)}}, \vec{q}\right)$, where $T^{\varepsilon(i)}=\top$ if $\varepsilon(i)=1$ and $T^{\varepsilon(i)}=\perp$ if $\varepsilon(i)=\partial$.
} 
TABLE 1. Skeleton and PIA nodes for DLE.

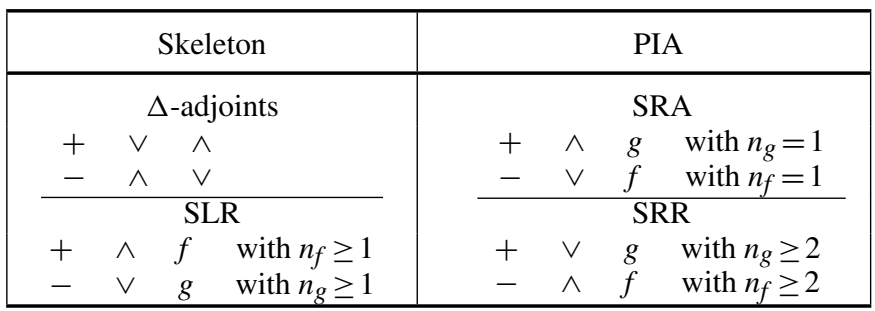
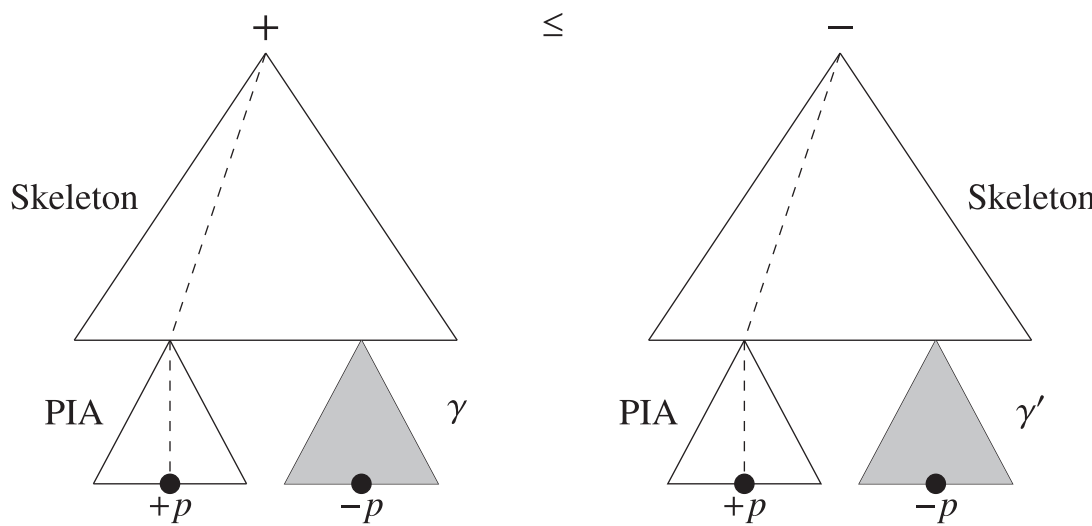

FIGURE 1. A schematic representation of inductive inequalities.

given in Table 1. A branch in a signed generation tree $* s$, with $* \in\{+,-\}$, is called a good branch if it is the concatenation of two paths $P_{1}$ and $P_{2}$, one of which may possibly be of length 0 , such that $P_{1}$ is a path from the leaf consisting (apart from variable nodes) only of PIA-nodes, ${ }^{23}$ and $P_{2}$ consists (apart from variable nodes) only of Skeleton nodes (for the definition of PIA- and Skeleton nodes see again table 1).

DEFINITION 16 (Inductive inequalities)

For any order type $\varepsilon$ and any irreflexive and transitive relation $<_{\Omega}$ on $p_{1}, \ldots p_{n}$, the signed generation tree $* s(* \in\{-,+\})$ of a term $s\left(p_{1}, \ldots p_{n}\right)$ is $(\Omega, \varepsilon)$-inductive if

(1) for all $1 \leq i \leq n$, every $\varepsilon$-critical branch with leaf $p_{i}$ is good (cf. Definition 15);

(2) every $m$-ary SRR-node occurring in the critical branch is of the form $\circledast\left(\gamma_{1}, \ldots, \gamma_{j-1}, \beta, \gamma_{j+1} \ldots, \gamma_{m}\right)$, where for any $h \in\{1, \ldots, m\} \backslash j$ :

(a) $\varepsilon^{\partial}\left(\gamma_{h}\right) \prec * s$ (cf. discussion before Definition 15), and

(b) $p_{k}<\Omega p_{i}$ for every $p_{k}$ occurring in $\gamma_{h}$ and for every $1 \leq k \leq n$.

We will refer to $<_{\Omega}$ as the dependency order on the variables. An inequality $s \leq t$ is $(\Omega, \varepsilon)$-inductive if the signed generation trees $+s$ and $-t$ are $(\Omega, \varepsilon)$-inductive. An inequality $s \leq t$ is inductive if it is $(\Omega, \varepsilon)$-inductive for some $\Omega$ and $\varepsilon$.

\footnotetext{
${ }^{23}$ For explanations of our choice of terminologies here, we refer to [46, Remark 3.24].
} 
In what follows, we will find it useful to refer to formulas $\phi$ such that only PIA nodes occur in $+\phi$ (resp. $-\phi$ ) as positive (resp. negative) PIA-formulas, and to formulas $\xi$ such that only Skeleton nodes occur in $+\xi$ (resp. $-\xi$ ) as positive (resp. negative) Skeleton formulas.

The proof of the following theorem is a straightforward generalization of [18, Theorem 10.11], and hence its proof is omitted.

\section{THEOREM 17}

For any language $\mathcal{L}_{\text {DLE }}$, its corresponding version of ALBA succeeds on all inductive $\mathcal{L}_{\mathrm{DLE}^{-}}$ inequalities, which are hence canonical ${ }^{24}$ and their corresponding logics are complete w.r.t. the elementary classes of relational structures ${ }^{25}$ defined by their first-order correspondents.

\subsection{The algorithm ALBA for $\mathcal{L}_{\mathrm{DLE}}$-inequalities}

The present subsection reports on the rules and execution of the algorithm ALBA in the setting of $\mathcal{L}_{\text {DLE }}$. ALBA manipulates inequalities and quasi-inequalities ${ }^{26}$ in the expanded language $\mathcal{L}_{\text {DLE }}^{*+}$, which is built up on the base of the lattice constants $T, \perp$ and an enlarged set of propositional variables NOM $\cup$ CONOM $\cup$ AtProp (the variables $\mathbf{i}, \mathbf{j}$ in $\mathrm{NOM}$ are referred to as nominals, and the variables $\mathbf{m}, \mathbf{n}$ in CONOM as conominals), closing under the logical connectives of $\mathcal{L}_{\mathrm{DLE}}^{*}$. The natural semantic environment of $\mathcal{L}_{\text {DLE }}^{*+}$ is given by perfect $\mathcal{L}_{\mathrm{DLE}}$-algebras. As already mentioned in the proof of Theorem 12, these algebras are endowed with a natural structure of bi-intuitionistic 'tense' $\mathcal{L}_{\mathrm{DLE}}$-algebra. Moreover, crucially, perfect $\mathcal{L}_{\mathrm{DLE}}$-algebras are both completely join-generated by their completely join-irreducible elements and completely meet-generated by their completely meet-irreducible elements. ${ }^{27}$ This property plays an important part in the algebraic account of the correspondence mechanism (cf. discussion in [16, Section 1.4]). Nominals and conominals, respectively, range over the sets of the completely join-irreducible elements and the completely meet-irreducible elements of perfect DLEs.

The version of ALBA relative to $\mathcal{L}_{\text {DLE }}$ runs as detailed in [18]. In a nutshell, $\mathcal{L}_{\text {DLE-inequalities }}$ are equivalently transformed into the conjunction of one or more $\mathcal{L}_{\text {DLE }}^{*+}$ quasi-inequalities, with the aim of eliminating propositional variable occurrences via the application of Ackermann rules. We refer the reader to [18] for details. In what follows, we illustrate how ALBA works, while at the same time we introduce its rules. The proof of the soundness and invertibility of the general rules for the DLE-setting is similar to the one provided in $[16,18]$. ALBA manipulates input inequalities $\phi \leq \psi$ and proceeds in three stages:

\subsubsection{First stage: preprocessing and first approximation}

ALBA preprocesses the input inequality $\phi \leq \psi$ by performing the following steps exhaustively in the signed generation trees $+\phi$ and $-\psi$ :

\footnotetext{
${ }^{24} \mathrm{An} \mathcal{L}_{\mathrm{DLE}}$-inequality $s \leq t$ is canonical if the class of $\mathcal{L}_{\mathrm{DLE}}$-algebras defined by $s \leq t$ is closed under the construction of canonical extension (cf. Footnote 19).

${ }^{25}$ Such are those introduced in $[54,55]$.

${ }^{26} \mathrm{~A}$ quasi-inequality of $\mathcal{L}_{\mathrm{DLE}}$ is an expression of the form $\mathcal{Q}_{i=1}^{n} s_{i} \leq t_{i} \Rightarrow s \leq t$, where $s_{i} \leq t_{i}$ and $s \leq t$ are $\mathcal{L}_{\mathrm{DLE}}$-inequalities for each $i$.

${ }^{27}$ A distributive lattice is perfect if it is complete, completely distributive and completely join-generated by the collection of its completely join-prime elements. Equivalently, a distributive lattice is perfect iff it is isomorphic to the lattice of upsets of some poset. A normal DLE is perfect if $D$ is a perfect distributive lattice, and each $f$-operation (resp. $g$-operation) is completely join-preserving (resp. meet-preserving) or completely meet-reversing (resp. join-reversing) in each coordinate.
} 
(1) (a) Push down, towards variables, occurrences of $+\wedge$, by distributing each of them over their children nodes labelled with $+\vee$ which are not in the scope of PIA nodes;

(b) Push down, towards variables, occurrences of $-\vee$, by distributing each of them over their children nodes labelled with $-\wedge$ which are not in the scope of PIA nodes;

(c) Push down, towards variables, occurrences of $+f$ for any $f \in \mathcal{F}$, by distributing each such occurrence over its $i$-th child node whenever the child node is labelled with $+\vee($ resp. $-\wedge)$ and is not in the scope of PIA nodes, and whenever $\varepsilon_{f}(i)=1$ (resp. $\varepsilon_{f}(i)=\partial$ );

(d) Push down, towards variables, occurrences of $-g$ for any $g \in \mathcal{G}$, by distributing each such occurrence over its $i$-th child node whenever the child node is labelled with $-\wedge($ resp. $+\vee)$ and is not in the scope of PIA nodes, and whenever $\varepsilon_{g}(i)=1$ (resp. $\varepsilon_{g}(i)=\partial$ ).

(2) Apply the splitting rules:

$$
\frac{\alpha \leq \beta \wedge \gamma}{\alpha \leq \beta \quad \alpha \leq \gamma} \quad \frac{\alpha \vee \beta \leq \gamma}{\alpha \leq \gamma \quad \beta \leq \gamma}
$$

(3) Apply the monotone and antitone variable-elimination rules:

$$
\frac{\alpha(p) \leq \beta(p)}{\alpha(\perp) \leq \beta(\perp)} \quad \frac{\beta(p) \leq \alpha(p)}{\beta(\top) \leq \alpha(\top)}
$$

for $\beta(p)$ positive in $p$ and $\alpha(p)$ negative in $p$.

\section{REMARK 18}

The standard ALBA preprocessing can be supplemented with the application of additional rules which replace SLR-nodes (resp. SRR-nodes) of the form $\circledast\left(\gamma_{1}, \ldots, \perp_{\circledast}^{\varepsilon_{\circledast}(i)}, \ldots, \gamma_{m}\right)$ (resp. $\left.\circledast\left(\gamma_{1}, \ldots, \top^{\varepsilon_{\circledast}}(i), \ldots, \gamma_{m}\right)\right)$ with $\perp$ (resp. $\left.\top\right)$. Although clearly sound, these rules have not been included in other ALBA settings such as [14, 18], since they are not strictly needed for the computation of first-order correspondents. However, in the present setting, ALBA is used for a different purpose than the one it was originally designed for. Allowing these rules to be applied during the preprocessing will address the problem of the occurrences of constants in the 'wrong' position, ${ }^{28}$ since it allows to transform e.g. a problematic premise into a tautology and make it hence disappear. These ideas will be expanded on in Sections 6.2 and 7.1.

Another step of the preprocessing which, although sound, is not included in standard executions of ALBA concerns the exhaustive application of the following distribution rules:

(a') Push down, towards variables, occurrences of $-\wedge$ in the scope of PIA-nodes which are not Skeleton nodes, by distributing each of them over their children nodes labelled with $-\vee$;

(b') Push down, towards variables, occurrences of $+\vee$ in the scope of PIA-nodes which are not Skeleton nodes, by distributing each of them over their children nodes labelled with $+\wedge$;

(c') Push down, towards variables, occurrences of $-f$ for any $f \in \mathcal{F}$, by distributing each such occurrence over its $i$-th child node whenever the child node is labelled with $-\vee($ resp. $+\wedge)$, and whenever $\varepsilon_{f}(i)=1$ (resp. $\varepsilon_{f}(i)=\partial$ );

(d') Push down, towards variables, occurrences of $+g$ for any $g \in \mathcal{G}$, by distributing each such occurrence over its $i$-th child node whenever the child node is labelled with $+\wedge$ (resp. $-\vee)$, and whenever $\varepsilon_{g}(i)=1$ (resp. $\varepsilon_{g}(i)=\partial$ ).

\footnotetext{
${ }^{28} \mathrm{As}$ we will see, in the context of analytic inductive inequalities, occurrences of $+\perp$ or $-\top$ as skeleton nodes and occurrences of $-\perp$ or $+\top$ as PIA-nodes are problematic. Indeed, in the context of the procedure which transforms inequalities into equivalent structural rules (cf. Sections 6 and 7.1), these logical constants would occur within certain sequents in positions (antecedent or succedent) in which they are not the interpretation of the corresponding structural constant. This fact would block the smooth transformation of logical axioms containing them into structural rules.
} 
Applied to PIA-terms, this additional step has the effect of surfacing all occurrences of $+\wedge$ and $-\vee$ up to the root of each PIA-term (so as to form a connected block of nodes including the root which are all labelled $+\wedge$ or all labelled $-\vee$ ). In this position, these occurrences can be all regarded as Skeleton nodes. Hence, after this step, no occurrences of $+\wedge$ and $-\vee$ will remain in the PIA subterms. ${ }^{29}$ Notice that applying this step to $(\Omega, \varepsilon)$-inductive terms produces $(\Omega, \varepsilon)$-inductive terms each PIA-subterm of which contains at most one $\varepsilon$-critical variable occurrence.

Let Preprocess $(\phi \leq \psi)$ be the finite set $\left\{\phi_{i} \leq \psi_{i} \mid 1 \leq i \leq n\right\}$ of inequalities obtained after the exhaustive application of the previous rules. We proceed separately on each of them, and hence, in what follows, we focus only on one element $\phi_{i} \leq \psi_{i}$ in Preprocess $(\phi \leq \psi)$, and we drop the subscript. Next, the following first approximation rule is applied only once to every inequality in $\operatorname{Preprocess}(\phi \leq \psi)$ :

$$
\frac{\phi \leq \psi}{\mathbf{i}_{0} \leq \phi \quad \psi \leq \mathbf{m}_{0}}
$$

Here, $\mathbf{i}_{0}$ and $\mathbf{m}_{0}$ are a nominal and a conominal, respectively. The first-approximation step gives rise to systems of inequalities $\left\{\mathbf{i}_{0} \leq \phi_{i}, \psi_{i} \leq \mathbf{m}_{0}\right\}$ for each inequality in $\operatorname{Preprocess}(\phi \leq \psi)$. Each such system is called an initial system, and is now passed on to the reduction-elimination cycle.

\subsubsection{Second stage: reduction-elimination cycle}

The goal of the reduction-elimination cycle is to eliminate all propositional variables from the systems received from the preprocessing phase. The elimination of each variable is effected by an application of one of the Ackermann rules given below. In order to apply an Ackermann rule, the system must have a specific shape. The adjunction, residuation, approximation, and splitting rules are used to transform systems into this shape. The rules of the reduction-elimination cycle, viz. the adjunction, residuation, approximation, splitting, and Ackermann rules, will be collectively called the reduction rules.

Residuation rules. Here below we provide the residuation rules relative to each $f \in \mathcal{F}$ and $g \in \mathcal{G}$ of arity at least 1 : for each $1 \leq h \leq n_{f}$ and each $1 \leq k \leq n_{g}$ :

$$
\begin{array}{llll}
\left(\varepsilon_{f}(h)=1\right) \frac{f\left(\psi_{1}, \ldots, \psi_{h}, \ldots, \psi_{n_{f}}\right) \leq \chi}{\psi_{h} \leq f_{h}^{\sharp}\left(\psi_{1}, \ldots, \chi, \ldots, \psi_{n_{f}}\right)} & & \frac{f\left(\psi_{1}, \ldots, \psi_{h}, \ldots, \psi_{n_{f}}\right) \leq \chi}{f_{h}^{\sharp}\left(\psi_{1}, \ldots, \chi, \ldots, \psi_{n_{f}}\right) \leq \psi_{h}}\left(\varepsilon_{f}(h)=\partial\right) \\
\left(\varepsilon_{g}(k)=\partial\right) \frac{\chi \leq g\left(\psi_{1}, \ldots, \psi_{k}, \ldots, \psi_{n_{g}}\right)}{\psi_{k} \leq g_{k}^{b}\left(\psi_{1}, \ldots, \chi, \ldots, \psi_{n_{g}}\right)} & \frac{\chi \leq g\left(\psi_{1}, \ldots, \psi_{k}, \ldots, \psi_{n_{g}}\right)}{g_{k}^{b}\left(\psi_{1}, \ldots, \chi, \ldots, \psi_{n_{g}}\right) \leq \psi_{k}}\left(\varepsilon_{g}(k)=1\right)
\end{array}
$$

Approximation rules. Here below we provide the approximation rules ${ }^{30}$ relative to each $f \in \mathcal{F}$ and $g \in \mathcal{G}$ of arity at least 1 : for each $1 \leq h \leq n_{f}$ and each $1 \leq k \leq n_{g}$,

\footnotetext{
${ }^{29}$ PIA subterms $* s$ in which no nodes $+\wedge$ and $-\vee$ occur are referred to as definite.

${ }^{30}$ The version of the approximation rules given in $[18,22,46]$ is slightly different from but equivalent to that of the approximation rules reported on here. That formulation is motivated by the need of enforcing the invariance of certain topological properties for the purpose of proving the canonicity of the inequalities on which ALBA succeeds. In this context, we do not need to take these constraints into account, and hence we can take this more flexible version of the approximation rules as primitive, bearing in mind that when proving canonicity one has to take a formulation analogous to that in [18, 22, 46] as primitive.
} 


$$
\begin{aligned}
& \left(\varepsilon_{f}(h)=1\right) \frac{\mathbf{i} \leq f\left(\psi_{1}, \ldots, \psi_{h}, \ldots, \psi_{n_{f}}\right)}{\mathbf{i} \leq f\left(\psi_{1}, \ldots, \mathbf{j}, \ldots, \psi_{n_{f}}\right) \quad \mathbf{j} \leq \psi_{h}} \quad \frac{g\left(\psi_{1}, \ldots, \psi_{k}, \ldots, \psi_{n_{g}}\right) \leq \mathbf{m}}{g\left(\psi_{1}, \ldots, \mathbf{n}, \ldots, \psi_{n_{g}}\right) \leq \mathbf{m} \quad \psi_{k} \leq \mathbf{n}}\left(\varepsilon_{g}(k)=1\right) \\
& \left(\varepsilon_{f}(h)=\partial\right) \frac{\mathbf{i} \leq f\left(\psi_{1}, \ldots, \psi_{h}, \ldots, \psi_{n_{f}}\right)}{\mathbf{i} \leq f\left(\psi_{1}, \ldots, \mathbf{n}, \ldots, \psi_{n_{f}}\right) \quad \psi_{k} \leq \mathbf{n}} \quad \frac{g\left(\psi_{1}, \ldots, \psi_{k}, \ldots, \psi_{n_{g}}\right) \leq \mathbf{m}}{g\left(\psi_{1}, \ldots, \mathbf{j}, \ldots, \psi_{n_{g}}\right) \leq \mathbf{m} \quad \mathbf{j} \leq \psi_{h}}\left(\varepsilon_{g}(k)=\partial\right)
\end{aligned}
$$

where the variable $\mathbf{j}$ (resp. $\mathbf{n}$ ) is a nominal (resp. a conominal). The nominals and conominals introduced by the approximation rules must be fresh, i.e. must not already occur in the system before applying the rule.

Ackermann rules. These rules are the core of ALBA, since their application eliminates proposition variables. As mentioned earlier, all the preceding steps are aimed at equivalently rewriting the input system into one of a shape in which the Ackermann rules can be applied. An important feature of Ackermann rules is that they are executed on the whole set of inequalities in which a given variable occurs, and not on a single inequality.

$$
\frac{\&\left\{\alpha_{i} \leq p \mid 1 \leq i \leq n\right\} \& \&\left\{\beta_{j}(p) \leq \gamma_{j}(p) \mid 1 \leq j \leq m\right\} \Rightarrow \mathbf{i} \leq \mathbf{m}}{\&\left\{\beta_{j}\left(\bigvee_{i=1}^{n} \alpha_{i}\right) \leq \gamma_{j}\left(\bigvee_{i=1}^{n} \alpha_{i}\right) \mid 1 \leq j \leq m\right\} \Rightarrow \mathbf{i} \leq \mathbf{m}}(\mathrm{RAR})
$$

where $p$ does not occur in $\alpha_{1}, \ldots, \alpha_{n}, \beta_{1}(p), \ldots, \beta_{m}(p)$ are positive in $p$, and $\gamma_{1}(p), \ldots, \gamma_{m}(p)$ are negative in $p$.

$$
\frac{\&\left\{p \leq \alpha_{i} \mid 1 \leq i \leq n\right\} \& \&\left\{\beta_{j}(p) \leq \gamma_{j}(p) \mid 1 \leq j \leq m\right\} \Rightarrow \mathbf{i} \leq \mathbf{m}}{\&\left\{\beta_{j}\left(\bigwedge_{i=1}^{n} \alpha_{i}\right) \leq \gamma_{j}\left(\bigwedge_{i=1}^{n} \alpha_{i}\right) \mid 1 \leq j \leq m\right\} \Rightarrow \mathbf{i} \leq \mathbf{m}}(\mathrm{LAR})
$$

where $p$ does not occur in $\alpha_{1}, \ldots, \alpha_{n}, \beta_{1}(p), \ldots, \beta_{m}(p)$ are negative in $p$, and $\gamma_{1}(p), \ldots, \gamma_{m}(p)$ are positive in $p$.

\subsubsection{Third stage: output}

If there was some system in the second stage from which not all occurring propositional variables could be eliminated through the application of the reduction rules, then ALBA reports failure and terminates. Else, each system $\left\{\mathbf{i}_{0} \leq \phi_{i}, \psi_{i} \leq \mathbf{m}_{0}\right\}$ obtained from Preprocess $(\varphi \leq \psi)$ has been reduced to a system, denoted Reduce $\left(\varphi_{i} \leq \psi_{i}\right)$, containing no propositional variables. Let $\operatorname{ALBA}(\varphi \leq \psi)$ be the set of quasi-inequalities

$$
\&\left[\operatorname{Reduce}\left(\varphi_{i} \leq \psi_{i}\right)\right] \Rightarrow \mathbf{i}_{0} \leq \mathbf{m}_{0}
$$

for each $\varphi_{i} \leq \psi_{i} \in \operatorname{Preprocess}(\varphi \leq \psi)$.

Notice that all members of $\operatorname{ALBA}(\varphi \leq \psi)$ are free of propositional variables. ALBA returns $\operatorname{ALBA}(\varphi \leq \psi)$ and terminates. An inequality $\varphi \leq \psi$ on which ALBA succeeds will be called an ALBA-inequality.

The proof of the following theorem is a straightforward generalization of [18, Theorem 8.1], and hence its proof is omitted.

THEOREM 19 (Correctness)

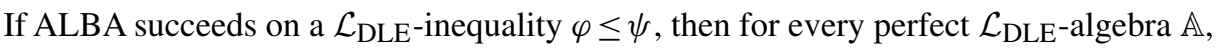

$$
\mathbb{A} \models \varphi \leq \psi \quad \text { iff } \quad \mathbb{A}=\operatorname{ALBA}(\varphi \leq \psi)
$$




\section{Display calculi for $L_{D L E}$ and $L_{D L E}^{*}$}

In the present section, we introduce the basic proof-theoretic environment of our treatment, given by the display calculi $\mathbf{D L}{ }^{*}$ and $\mathbf{D L}$ for the logics $\mathbf{L}_{\mathrm{DLE}}$ and $\mathbf{L}_{\mathrm{DLE}}^{*}$ associated with any given language $\mathcal{L}_{\text {DLE }}(\mathcal{F}, \mathcal{G})$. We also show some of their basic properties.

\subsection{Language and rules}

The present subsection is aimed at simultaneously introducing the display calculi $\mathbf{D L} \mathbf{L}^{*}$ and $\mathbf{D L}$ for $\mathbf{L}_{\text {DLE }}^{*}$ and $\mathbf{L}_{\text {DLE }}$, respectively. As is usual of existing logical systems which the present framework intends to capture (e.g. intuitionistic and bi-intuitionistic logics, or modal and tense logics [36]), the languages manipulated by these calculi are built up using one and the same set of structural terms, and differ only in the set of operational term constructors. In the tables below, each structural symbol in the upper rows corresponds to one or two logical (or operational) symbols. The idea, which will be made precise later on, is that each structural connective can be interpreted as the corresponding left-hand (resp. right-hand) side logical connective (if it exists) when occurring in antecedent (resp. consequent) position.

- Structural symbols for lattice operators:

\begin{tabular}{|r|c|c|c|c|c|c|c|c|}
\hline Structural symbols & \multicolumn{2}{|c|}{$\mathrm{I}$} & \multicolumn{2}{c|}{$;$} & \multicolumn{2}{c|}{$>$} & \multicolumn{2}{c|}{$<$} \\
\hline Operational symbols & $\top$ & $\perp$ & $\wedge$ & $\vee$ & $(\succ)$ & $(\rightarrow)$ & $(\smile)$ & $(\leftarrow)$ \\
\hline
\end{tabular}

- Structural symbols for any $f \in \mathcal{F}$ and $g \in \mathcal{G}$ :

\begin{tabular}{|r|r|l|l|l|}
\hline Structural symbols & \multicolumn{2}{|c|}{$H$} & \multicolumn{2}{c|}{$K$} \\
\hline Operational symbols & $f$ & & & $g$ \\
\hline
\end{tabular}

- Structural symbols for any $f_{i}^{\sharp}, g_{h}^{b} \in\left(\mathcal{F}^{*} \cup \mathcal{G}^{*}\right) \backslash(\mathcal{F} \cup \mathcal{G})$, and any $0 \leq i \leq n_{f}$ and $0 \leq h \leq n_{g}$ :

\begin{tabular}{|c|c|c|c|c|}
\hline Structural symbols & $H_{i}\left(\varepsilon_{f}(i)=1\right)$ & $H_{i}\left(\varepsilon_{f}(i)=\partial\right)$ & $K_{h}\left(\varepsilon_{g}(h)=1\right)$ & $K_{h}\left(\varepsilon_{g}(h)=\partial\right)$ \\
\hline Operational symbols & \begin{tabular}{l|l} 
& $\left(f_{i}^{\sharp}\right)$
\end{tabular} & \begin{tabular}{l|l}
$\left(f_{i}^{\sharp}\right)$ &
\end{tabular} & \begin{tabular}{l|l}
$\left(g_{h}^{b}\right)$ &
\end{tabular} & \begin{tabular}{l|l} 
& $\left(g_{h}^{b}\right)$
\end{tabular} \\
\hline
\end{tabular}

Some operational symbols above appear in brackets as a reminder that, unlike their associated structural symbols, they might occur only in the language and in the calculus for $\mathbf{L}_{\mathrm{DLE}}^{*}$.

REMARK 20

If $f \in \mathcal{F}$ and $g \in \mathcal{G}$ form a dual pair, ${ }^{31}$ then $n_{f}=n_{g}$ and $\varepsilon_{f}=\varepsilon_{g}$. Then $f$ and $g$ can be assigned one and the same structural operator, as follows:

\begin{tabular}{|c|c|c|}
\hline Structural symbols & \multicolumn{2}{|c|}{$H$} \\
\hline Operational symbols & $f$ & $g$ \\
\hline
\end{tabular}

\footnotetext{
${ }^{31}$ Examples of dual pairs are $(\top, \perp),(\wedge, \vee),(\succ, \rightarrow),(\prec, \leftarrow)$, and $(\diamond, \square)$ where $\diamond$ is defined as $\neg \square \neg$.
} 
Moreover, for any $1 \leq i \leq n_{f}=n_{g}$, the residuals $f_{i}^{\sharp}$ and $g_{i}^{b}$ are dual to one another. Hence, they can also be assigned one and the same structural connective as follows:

\begin{tabular}{|r|c|c|c|c|}
\hline Structural symbols & \multicolumn{2}{|c|}{$H_{i}\left(\varepsilon_{f}(i)=\varepsilon_{g}(i)=1\right)$} & \multicolumn{2}{c|}{$H_{i}\left(\varepsilon_{f}(i)=\varepsilon_{g}(i)=\partial\right)$} \\
\hline Operational symbols & $\left(g_{i}^{\mathrm{b}}\right)$ & $\left(f_{i}^{\sharp}\right)$ & $\left(f_{i}^{\sharp}\right)$ & $\left(g_{i}^{\mathrm{b}}\right)$ \\
\hline
\end{tabular}

\section{DEFINITION 21}

The display calculi DL* and DL consist of the following display postulates, structural rules, and operational rules ${ }^{32}$ :

(1) Identity and cut:

$$
p \vdash p \quad \frac{X \vdash A \quad A \vdash Y}{X \vdash Y}
$$

(2) Display postulates for lattice connectives:

$$
\frac{X ; Y \vdash Z}{Y \vdash X>Z} \quad \frac{Z \vdash X ; Y}{\overline{X>Z \vdash Y}} \quad \frac{X ; Y \vdash Z}{X \vdash Z<Y} \quad \frac{Z \vdash X ; Y}{\overline{Z<Y \vdash X}}
$$

(3) Display postulates for $f \in \mathcal{F}$ and $g \in \mathcal{G}$ : for any $1 \leq i \leq n_{f}$ and $1 \leq h \leq n_{g}$,

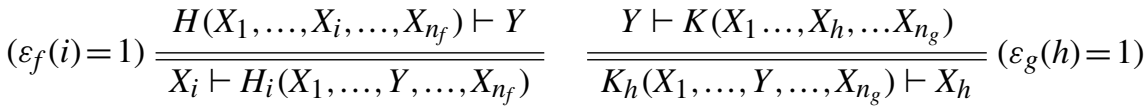

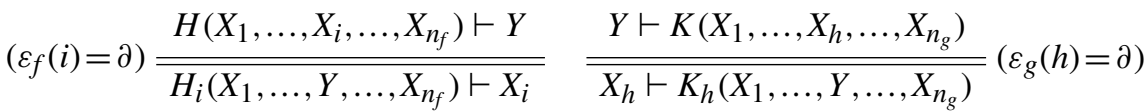

Notice that the display postulates for all the connectives in $\mathcal{F}^{*} \cup \mathcal{G}^{*}$ are derivable from the display postulates above. The rules for the case of connectives in the dual pairs are obtained by replacing $K$ for $H$ in the corresponding rules above.

(4) Necessitation for $f \in \mathcal{F}$ and $g \in \mathcal{G}$ : for any $1 \leq k \leq n_{f}$ and $1 \leq h \leq n_{g}$,

$$
\begin{array}{lll}
\left.\left(\varepsilon_{f}(k)=1\right) \frac{\left(X_{i} \vdash Y_{i}\right.}{Y_{j} \vdash X_{j} \mid i \neq k, 1 \leq i, j \leq n_{f}, \varepsilon_{f}(i)=1 \text { and } \varepsilon_{f}(j)=\partial}\right) & X_{k} \vdash \mathrm{I}_{k} \\
X_{k} \vdash H_{k}\left(X_{1}, \ldots, X_{k-1}, \mathrm{I}, X_{k+1}, \ldots, X_{n_{f}}\right) & \\
\left(\varepsilon_{f}(k)=\partial\right) \frac{\left(X_{i} \vdash Y_{i}\right.}{\left.Y_{j} \vdash X_{j} \mid j \neq k, 1 \leq i, j \leq n_{f}, \varepsilon_{f}(i)=1 \text { and } \varepsilon_{f}(j)=\partial\right)} & \mathrm{I}_{k} \vdash X_{k} \\
H_{k}\left(X_{1}, \ldots, X_{k-1}, \mathrm{I}, X_{k+1}, \ldots, X_{n_{f}}\right) \vdash X_{k} &
\end{array}
$$

\footnotetext{
${ }^{32}$ The display calculus associated with the basic DLE-logic $\mathbf{L}^{*}$ (cf. Footnote 17) in the expanded language $\mathcal{L}_{\text {DLE }}^{*}$ is denoted by $\mathbf{D L}^{*}$, and is defined by instantiating the definition of $\mathbf{D L}$ to the expanded language $\mathcal{L}_{\mathrm{DLE}}^{*}$.
} 


$$
\begin{array}{ccc}
\left.\left(\varepsilon_{g}(h)=1\right) \frac{\left(X_{j} \vdash Y_{j}\right.}{Y_{i} \vdash X_{i} \mid i \neq h, 1 \leq i, j \leq n_{f}, \varepsilon_{f}(i)=1 \text { and } \varepsilon_{f}(j)=\partial}\right) & \mathrm{I}_{h} \vdash X_{h} \\
K_{h}\left(X_{1}, \ldots, X_{h-1}, \mathrm{I}, X_{h+1}, \ldots, X_{n_{g}}\right) \vdash X_{h} & \\
\left(\varepsilon_{g}(h)=\partial\right) \frac{\left(X_{j} \vdash Y_{j}\right.}{\left.Y_{i} \vdash X_{i} \mid j \neq h, 1 \leq i, j \leq n_{f}, \varepsilon_{f}(i)=1 \text { and } \varepsilon_{f}(j)=\partial\right)} & X_{h} \vdash \mathrm{I}_{h} \\
X_{h} \vdash K_{h}\left(X_{1}, \ldots, X_{h-1}, \mathrm{I}, X_{h+1}, \ldots, X_{n_{g}}\right) &
\end{array}
$$

(5) Structural rules encoding the distributive lattice axiomatization:

$$
\begin{aligned}
& \mathrm{I}_{L} \frac{X \vdash Y}{\overline{\mathrm{I} ; X \vdash Y}} \quad \frac{Y \vdash X}{Y \vdash X ; \mathrm{I}} \mathrm{I}_{R} \quad E_{L} \frac{Y ; X \vdash Z}{X ; Y \vdash Z} \quad \frac{Z \vdash X ; Y}{Z \vdash Y ; X} E_{R} \\
& W_{L} \frac{Y \vdash Z}{X ; Y \vdash Z} \quad \frac{Z \vdash Y}{Z \vdash Y ; X} W_{R} \quad C_{L} \frac{X ; X \vdash Y}{X \vdash Y} \quad \frac{Y \vdash X ; X}{Y \vdash X} C_{R}
\end{aligned}
$$

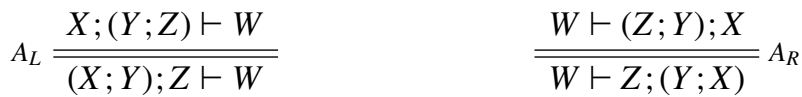

(6) Introduction rules for the propositional (BDL and bi-intuitionistic) connectives:

$$
\begin{aligned}
& \perp_{L} \frac{X \vdash \mathrm{I}}{\perp \vdash \mathrm{I}} \quad \perp_{R} \quad \mathrm{~T}_{L} \frac{\mathrm{I} \vdash X}{\mathrm{~T} \vdash X} \quad \stackrel{ }{\mathrm{I} \vdash \mathrm{T}} \mathrm{T}_{R} \\
& \wedge_{L} \frac{A ; B \vdash X}{A \wedge B \vdash X} \quad \frac{X \vdash A \quad Y \vdash B}{X ; Y \vdash A \wedge B} \wedge_{R} \quad \vee_{L} \frac{A \vdash X \quad B \vdash Y}{A \vee B \vdash X ; Y} \quad \frac{X \vdash A ; B}{X \vdash A \vee B} \vee_{R} \\
& \rightarrow_{L} \frac{X \vdash A \quad B \vdash Y}{A \rightarrow B \vdash X>Y} \quad \frac{X \vdash A>B}{X \vdash A \rightarrow B} \rightarrow R \quad \quad>_{L} \frac{A>B \vdash Z}{A>B \vdash Z} \quad \frac{A \vdash X \quad Y \vdash B}{X>Y \vdash A>B}>>_{R} \\
& \leftarrow_{L} \frac{X \vdash A \quad B \vdash Y}{A \leftarrow B \vdash X<Y} \quad \frac{X \vdash A<B}{X \vdash A \leftarrow B} \leftarrow_{R} \quad \quad<_{L} \frac{A<B \vdash Z}{A<B \vdash Z} \quad \frac{A \vdash X \quad Y \vdash B}{X<Y \vdash A-B}<_{R}
\end{aligned}
$$

In the presence of the exchange rules $E_{L}$ and $E_{R}$, the structural connective $<$ and the corresponding operational connectives $<$ and $\leftarrow$ are redundant.

(7) Introduction rules for $f \in \mathcal{F}$ and $g \in \mathcal{G}$ :

$$
\begin{gathered}
f_{L} \frac{H\left(A_{1}, \ldots, A_{n_{f}}\right) \vdash X}{f\left(A_{1}, \ldots, A_{n_{f}}\right) \vdash X} \quad \frac{X \vdash K\left(A_{1}, \ldots, A_{n_{g}}\right)}{X \vdash g\left(A_{1}, \ldots, A_{n_{g}}\right)} g_{R} \\
f_{R} \frac{\left(X_{i} \vdash A_{i} \quad A_{j} \vdash X_{j} \mid 1 \leq i, j \leq n_{f}, \varepsilon_{f}(i)=1 \text { and } \varepsilon_{f}(j)=\partial\right)}{H\left(X_{1}, \ldots, X_{n_{f}}\right) \vdash f\left(A_{1}, \ldots, A_{n}\right)} \\
g_{L} \frac{\left(A_{i} \vdash X_{i} \quad X_{j} \vdash A_{j} \mid 1 \leq i, j \leq n_{g}, \varepsilon_{g}(i)=1 \text { and } \varepsilon_{g}(j)=\partial\right)}{g\left(A_{1}, \ldots, A_{n_{g}}\right) \vdash K\left(X_{1}, \ldots, X_{n}\right)}
\end{gathered}
$$

In particular, if $f$ and $g$ are 0 -ary (i.e. they are constants), the rules $f_{R}$ and $g_{L}$ above reduce to the axioms (0-ary rule) $H \vdash f$ and $g \vdash K$. 
(8) Only for $\mathrm{DL}^{*}$, introduction rules for each $f_{i}^{\sharp}, g_{h}^{b} \in\left(\mathcal{F}^{*} \cup \mathcal{G}^{*}\right) \backslash(\mathcal{F} \cup \mathcal{G})$ :

(a) If $\varepsilon_{f}(i)=1$ and $\varepsilon_{g}(h)=1$,

$$
\begin{gathered}
g_{h L}^{b} \frac{K_{h}\left(A_{1}, \ldots, A_{n_{g}}\right) \vdash X}{g_{h}^{b}\left(A_{1}, \ldots, A_{n_{f}}\right) \vdash X} \quad \frac{X \vdash H_{i}\left(A_{1}, \ldots, A_{n_{g}}\right)}{X \vdash f_{i}^{\sharp}\left(A_{1}, \ldots, A_{n_{g}}\right)} f_{i R}^{\sharp} \\
g_{h R}^{\mathrm{b}} \frac{\left(X_{\ell} \vdash A_{\ell} \quad A_{m} \vdash X_{m} \mid 1 \leq \ell, m \leq n_{g}, \varepsilon_{g_{h}^{b}}(\ell)=1 \text { and } \varepsilon_{g_{h}^{b}}(m)=\partial\right)}{K_{h}\left(X_{1}, \ldots, X_{n_{g}}\right) \vdash g_{h}^{b}\left(A_{1}, \ldots, A_{n_{g}}\right)} \\
f_{i_{L}}^{\sharp} \frac{\left(A_{\ell} \vdash X_{\ell} \quad X_{m} \vdash A_{m} \mid 1 \leq \ell, m \leq n_{g}, \varepsilon_{f_{i}^{\sharp}}(\ell)=1 \text { and } \varepsilon_{f_{i}^{\sharp}}(m)=\partial\right)}{f_{i}^{\sharp}\left(A_{1}, \ldots, A_{n_{g}}\right) \vdash H_{i}\left(X_{1}, \ldots, X_{n_{g}}\right)}
\end{gathered}
$$

(b) If $\varepsilon_{f}(i)=\partial$ and $\varepsilon_{g}(h)=\partial$,

$$
\begin{aligned}
& f_{i L}^{\sharp} \frac{H_{i}\left(A_{1}, \ldots, A_{n_{f}}\right) \vdash X}{f_{i}^{\sharp}\left(A_{1}, \ldots, A_{n_{f}}\right) \vdash X} \quad \frac{X \vdash K_{h}\left(A_{1}, \ldots, A_{n_{g}}\right)}{X \vdash g_{h}^{b}\left(A_{1}, \ldots, A_{n_{g}}\right)} g_{h R}^{b} \\
& f_{i R}^{\sharp} \frac{\left(X_{\ell} \vdash A_{\ell} \quad A_{m} \vdash X_{m} \mid 1 \leq \ell, m \leq n_{f}, \varepsilon_{f_{i}^{\sharp}}(\ell)=1 \text { and } \varepsilon_{f_{i}^{\sharp}}(m)=\partial\right)}{H_{i}\left(X_{1}, \ldots, X_{n_{f}}\right) \vdash f_{i}^{\sharp}\left(A_{1}, \ldots, A_{n_{f}}\right)} \\
& g_{h L}^{b} \frac{\left(A_{\ell} \vdash X_{\ell} \quad X_{m} \vdash A_{m} \mid 1 \leq \ell, m \leq n_{g}, \varepsilon_{g_{h}^{b}}(\ell)=1 \text { and } \varepsilon_{g_{h}^{\mathrm{b}}}(m)=\partial\right)}{g_{h}^{\mathrm{b}}\left(A_{1}, \ldots, A_{n_{g}}\right) \vdash K_{h}\left(X_{1}, \ldots, X_{n_{g}}\right)}
\end{aligned}
$$

A display calculus enjoys the full display property (resp. the relativized display property) if for every (derivable) sequent $X \vdash Y$ and every substructure $Z$ of either $X$ or $Y$, the sequent $X \vdash Y$ can be equivalently transformed, using the rules of the system, into a sequent which is either of the form $Z \vdash W$ or of the form $W \vdash Z$, for some structure $W$. A routine check will show that the display calculi DL and $\mathbf{D L}{ }^{*}$ both enjoy the relativized display property, and moreover, if $\mathcal{F}$ and $\mathcal{G}$ are such that for every $f \in \mathcal{F}$ the dual of $f$ is in $\mathcal{G}$ and for every $g \in \mathcal{G}$ the dual of $g$ is in $\mathcal{F}$, then DL and DL* both enjoy the full display property. The proof of these facts is omitted.

PROPOSITION 22

The display calculi DL and $\mathbf{D L} \mathbf{L}^{*}$ enjoy the relativized display property, and under the assumption above on $\mathcal{F}$ and $\mathcal{G}$ they enjoy the full display property.

\subsection{Soundness, completeness, conservativity}

\subsubsection{Soundness}

Let us expand on how to interpret structures and sequents in the language manipulated by the calculi DL and DL* in any perfect $\mathcal{L}_{\mathrm{DLE}}$-algebra $\mathbb{A}$ (cf. Footnote 27 ). Structures will be translated into formulas, and formulas will be interpreted as elements of $\mathbb{A}$. In order to translate structures as formulas, structural terms need to be translated as formulas, as is specified in Definition 24 below. To this effect, any given occurrence of a structural connective in a sequent is translated as (one or the other of) its associated logical connective(s), as reported in Table 2, provided its operational counterpart relative to its position (antecedent or succedent) exists. Clearly, not 
TABLE 2. Translation of structural connectives into logical connectives

\begin{tabular}{|c|c|c|c|}
\hline $\begin{array}{r}\text { Structural } \\
\text { connective }\end{array}$ & $\begin{array}{l}\text { if in precedent } \\
\text { position }\end{array}$ & $\begin{array}{l}\text { if in succedent } \\
\text { position }\end{array}$ & \\
\hline I & $\top$ & $\perp$ & \\
\hline$A ; B$ & $A \wedge B$ & $A \vee B$ & \\
\hline$A>B$ & $A>-B$ & $A \rightarrow B$ & \\
\hline$H(\bar{A})$ & $f(\bar{A})$ & & \\
\hline$K(\bar{A})$ & \multirow{5}{*}{$\begin{array}{c}f_{i}^{\sharp}(\bar{A}) \\
g_{h}^{b}(\bar{A})\end{array}$} & $g(\bar{A})$ & \\
\hline$H_{i}(\bar{A})$ & & $f_{i}^{\sharp}(\bar{A})$ & if $\varepsilon_{f}(i)=1$ \\
\hline$H_{i}(\bar{A})$ & & & if $\varepsilon_{f}(i)=\partial$ \\
\hline$K_{h}(\bar{A})$ & & & if $\varepsilon_{g}(h)=1$ \\
\hline$K_{h}(\bar{A})$ & & $g_{h}^{b}(\bar{A})$ & if $\varepsilon_{g}(h)=\partial$ \\
\hline
\end{tabular}

all structural terms will in general have a translation as formulas. This motivates the following definition:

\section{DEFINITION 23}

A structural term $S$ is left-sided (resp. right-sided) if in its positive (resp. negative) signed generation tree, ${ }^{33}$ every positive node is labelled with a structural connective which is associated with a logical connective when occurring in antecedent position, and every negative node is labelled with a structural connective which is associated with a logical connective when occurring in succedent position.

Clearly, if every structural connective is associated with some logical connectives both when occurring in antecedent position and when occurring in succedent position, as is the case e.g. when $\mathcal{F}$ and $\mathcal{G}$ bijectively correspond via conjugation, every structural term is both left-sided and right-sided.

\section{DEFINITION 24}

For every left-sided (resp. right-sided) structural term $S$, let $l(S)$ (resp. $r(S)$ ) denote the formula associated with $S$ and defined inductively according to Table 2.

Structural sequents $S \vdash T$ such that $S$ is left-sided and $T$ is right-sided are those translatable as formula-sequents $l(S) \vdash r(T)$. These sequents in turn are interpreted in any $\mathcal{L}_{\mathrm{DLE}}$-algebra $\mathbb{A}$ in the standard way. Hence, for any assignment $v: \operatorname{AtProp} \rightarrow \mathbb{A}$, we denote by $\mathbb{I} \cdot \mathbb{1}_{v}$ the unique homomorphic extension of $v$ to the formula algebra, interpret sequents $l(S) \vdash r(T)$ as inequalities $\llbracket l(S) \rrbracket_{v} \leq \llbracket r(T) \rrbracket_{v}$ and rules $\left(S_{i} \vdash T_{i} \mid i \in I\right) / S \vdash T$ as implications of the form 'if $\llbracket l\left(S_{i}\right) \rrbracket_{v} \leq \llbracket r\left(T_{i}\right) \rrbracket_{v}$ for every $i \in I$, then $\llbracket l(S) \rrbracket_{v} \leq \llbracket r(T) \rrbracket_{v}{ }^{\prime}$.

Under these stipulations, it is routine to check that all axioms and rules of the calculi DL and DL* are satisfied under any assignment. Hence, it is immediate to prove, by induction on the depth of the derivation tree, that

\section{PROPOSITION 25}

If $S \vdash T$ is DL-derivable (resp. DL*-derivable), then $S$ is left-sided, $T$ is right-sided and $\llbracket l(S) \rrbracket_{v} \leq$ $\llbracket r(T) \rrbracket_{v}$ is satisfied on every perfect $\mathcal{L}_{\mathrm{DLE}}$-algebra $\mathbb{A}$ and under any assignment $v:$ AtProp $\rightarrow \mathbb{A}$.

\footnotetext{
${ }^{33}$ Signed generation trees of structural terms are defined analogously to signed generation trees of logical terms. Logical formulas label the leaves of the signed generation trees of structural terms.
} 


\subsubsection{Completeness}

At the end of Section 3.2, we outlined the proof of the completeness of $\mathbf{L}_{\mathrm{DLE}}$ and $\mathbf{L}_{\text {DLE }}^{*}$ w.r.t. perfect

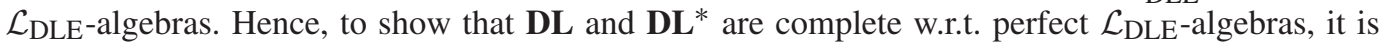
enough to show that the axioms and rules of $\mathbf{L}_{\mathrm{DLE}}$ (resp. $\mathbf{L}_{\mathrm{DLE}}^{*}$ ) are derivable in $\mathbf{D L}$ (resp. $\mathbf{D L} \mathbf{L}^{*}$ ). These verifications are routine. For instance, let $f \in \mathcal{F}$ be binary and s.t. $\varepsilon_{f}=(1, \partial)$. Then the following sequents are derivable in DL:

$$
\begin{array}{lr}
f_{1}^{\sharp}(A, C) \wedge f_{1}^{\sharp}(B, C) \vdash f_{1}^{\sharp}(A \wedge B, C) \quad f_{1}^{\sharp}(A, B) \wedge f_{1}^{\sharp}(A, C) \vdash f_{1}^{\sharp}(A, B \wedge C) \\
f_{2}^{\sharp}(A \vee B, C) \vdash f_{2}^{\sharp}(A, C) \vee f_{2}^{\sharp}(B, C) \quad f_{2}^{\sharp}(A, B \wedge C) \vdash f_{2}^{\sharp}(A, B) \vee f_{2}^{\sharp}(A, C) .
\end{array}
$$

By way of example, a derivation for $f_{1}^{\sharp}(A, C) \wedge f_{1}^{\sharp}(B, C) \vdash f_{1}^{\sharp}(A \wedge B, C)$ is reported below.

$$
\begin{array}{cr}
\frac{A \vdash A}{f_{1}^{\sharp}(A, C) \vdash H_{1}[A, C]} & \frac{B \vdash B}{f_{1}^{\sharp}(B, C) \vdash H_{1}[B, C]} \\
\frac{f_{1}^{\sharp}(A, C) ; f_{1}^{\sharp}(B, C) \vdash H_{1}[A, C]}{\frac{f_{1}^{\sharp}(A, C) \wedge f_{1}^{\sharp}(B, C) \vdash H_{1}[A, C]}{H\left[f_{1}^{\sharp}(A, C) \wedge f_{1}^{\sharp}(B, C), C\right] \vdash A}} & \frac{f_{1}^{\sharp}(A, C) ; f_{1}^{\sharp}(B, C) \vdash H_{1}[B, C]}{f_{1}^{\sharp}(A, C) \wedge f_{1}^{\sharp}(B, C) \vdash H_{1}[B, C]} \\
\frac{H\left[f_{1}^{\sharp}(A, C) \wedge f_{1}^{\sharp}(B, C), C\right] ; H\left[f_{1}^{\sharp}(A, C) \wedge f_{1}^{\sharp}(B, C), C\right] \vdash A \wedge B}{H\left[f_{1}^{\sharp}(A, C) \wedge f_{1}^{\sharp}(B, C), C\right] \vdash B} \\
\frac{H\left[f_{1}^{\sharp}(A, C) \wedge f_{1}^{\sharp}(B, C), C\right] \vdash A \wedge B}{f_{1}^{\sharp}(A, C) \wedge f_{1}^{\sharp}(B, C) \vdash H_{1}[A \wedge B, C]} \\
f_{1}^{\sharp}(A, C) \wedge f_{1}^{\sharp}(B, C) \vdash f_{1}^{\sharp}(A \wedge B, C)
\end{array}
$$

\subsubsection{Conservativity}

Let $A \vdash B$ be a $\mathbf{D L}^{*}$-derivable sequent in the language of $\mathbf{D L}$ (i.e. no operational connective in $\left(\mathcal{F}^{*} \cup\right.$ $\left.\mathcal{G}^{*}\right) \backslash(\mathcal{F} \cup \mathcal{G})$ occurs in the sequent). Hence, by the soundness of $\mathbf{D L}^{*}$ w.r.t. perfect $\mathcal{L}_{\mathrm{DLE}}$-algebras, the inequality $A \leq B$ is valid on these algebras. By the completeness of $\mathbf{L}_{\mathrm{DLE}}$ w.r.t. perfect $\mathcal{L}_{\mathrm{DLE}^{-}}$ algebras, the inequality $A \leq B$ is derivable in $\mathbf{L}_{\mathrm{DLE}}$, which implies, by the syntactic completeness of DL w.r.t. $\mathbf{L}_{\mathrm{DLE}}$, that $A \vdash B$ is $\mathbf{D L}$-derivable, as required.

\subsection{Cut-elimination and subformula property}

The calculi DL and $\mathbf{D L}{ }^{*}$ are proper display calculi, and hence, by Theorem 3, they enjoy Belnap-style cut-elimination and subformula property.

THEOREM 26

The calculi DL and $\mathbf{D L} *$ are proper display calculi.

Proof. The conditions $\mathrm{C}_{1}-\mathrm{C}_{7}$ can be straightforwardly verified by inspection on the rules. As to $\mathrm{C}_{8}$, cf. Fact 67 in the Appendix. 


\subsection{Properly displayable $\mathcal{L}_{\mathrm{DLE}}$-logics}

DEFINITION 27

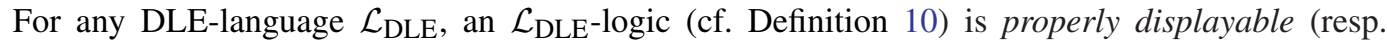
specially displayable) if it is exactly captured by a display calculus obtained by adding analytic rules (resp. special rules) — cf. Definition 4 (resp. Definition 6) — to the calculus DL for $\mathcal{L}_{\mathrm{DLE}}$.

\section{Primitive inequalities and special rules}

In [38, Theorem 16], Kracht showed that primitive formulas of basic normal/tense modal logic on a classical propositional base can be equivalently transformed into (a set of) special structural rules satisfying the defining conditions of proper display calculi (cf. Section 2.2). In the present section, we extend this result to any language $\mathcal{L}_{\text {DLE }}$. We base this extension on the notion of primitive inequalities. Namely, in Section 5.1, we introduce the class of (left- and right-)primitive inequalities in any language $\mathcal{L}_{\text {DLE }}$ (cf. Definition 28), and show (cf. Lemma 32) that these inequalities can be equivalently (and effectively) transformed into special structural rules (cf. in the restricted sense of Definition 6). We also show that special structural rules can be equivalently (and effectively) transformed into primitive inequalities. In Section 5.2, we identify the crucial order-theoretic feature induced by the syntactic shape of definite primitive inequalities (cf. Lemma 35), on the basis of which a special ALBA-type reduction for definite primitive inequalities is given (cf. Proposition 37). In Section 5.3, we take stock of the previous results and outline the way they will be further extended in Section 6.

\subsection{Left-primitive and right-primitive inequalities and special rules}

In what follows, for each connective $f \in \mathcal{F}$ and $g \in \mathcal{G}$, we will write $f(\vec{p}, \vec{q})$ and $g(\vec{p}, \vec{q})$, stipulating that $\varepsilon_{f}(p)=\varepsilon_{g}(p)=1$ for all $p$ in $\vec{p}$, and $\varepsilon_{f}(q)=\varepsilon_{g}(q)=\partial$ for all $q$ in $\vec{q}$. Moreover, we write e.g. $f(\vec{u} / \vec{p}, \vec{v} / \vec{q})$ to indicate that the arrays $\vec{u}$ and $\vec{p}$ (resp. $\vec{v}$ and $\vec{q}$ ) have the same length $n$ (resp. $m$ ) and that, for each $1 \leq i \leq n$ (resp. for each $1 \leq j \leq m$ ), the formula $u_{i}$ (resp. $v_{j}$ ) has been uniformly substituted in $f$ for the variable $p_{i}$ (resp. $q_{j}$ ).

DeFinition 28 (Primitive inequalities)

For any language $\mathcal{L}_{\mathrm{DLE}}=\mathcal{L}_{\mathrm{DLE}}(\mathcal{F}, \mathcal{G})$, the left-primitive $\mathcal{L}_{\mathrm{DLE}}$-formulas $\psi$ and right-primitive $\mathcal{L}_{\mathrm{DLE}^{-}}$ formulas $\phi$ are defined by simultaneous recursion as follows:

$$
\begin{aligned}
& \psi:=p|\top| \vee|\wedge| f(\vec{\psi} / \vec{p}, \vec{\phi} / \vec{q}), \\
& \phi:=p|\perp| \wedge|\vee| g(\vec{\phi} / \vec{p}, \vec{\psi} / \vec{q}) .
\end{aligned}
$$

A left-primitive (resp. right-primitive) $\mathcal{L}_{\mathrm{DLE}}$-formula is definite if there are no occurrences of $+\vee$ or $-\wedge\left(\right.$ resp. $+\wedge$ or $-\vee$ ) in its positive generation tree. An $\mathcal{L}_{\mathrm{DLE}}$-inequality $s_{1} \leq s_{2}$ is left-primitive (resp. right-primitive) if both $s_{1}$ and $s_{2}$ are left-primitive (resp. right-primitive) formulas and moreover:

(1) Each proposition variable in $s_{1}$ (resp. $s_{2}$ ) occurs at most once, in which case we say that $s_{1}$ (resp. $s_{2}$ ) is scattered.

(2) $s_{1}$ and $s_{2}$ have the same order type relative to the variables they have in common.

(3) $s_{2}$ (resp. $s_{1}$ ) is $\varepsilon$-uniform w.r.t. some order type $\varepsilon$ on its occurring variables.

Sometimes, the scattered side of a primitive inequality will be referred to as its head and the other one as its tail. 
It immediately follows from the axiomatization of the basic logic $\mathbf{L}_{\mathrm{DLE}}$ that left-primitive (resp. rightprimitive) $\mathcal{L}_{\mathrm{DLE}}$-formulas can be equivalently written in disjunction (resp. conjunction) normal form of definite left-primitive (resp. right-primitive) formulas. The condition that the head of a primitive inequality is scattered implies that the head is $\varepsilon$-uniform for the order type $\varepsilon$ of its occurring variables in e.g. its positive generation tree. Notice that the definition above does not exclude the possibility that some variables which do not occur in the head of a primitive inequality might occur in its tail. However, item (3) of the definition above requires the tail to be uniform in these variables. This observation will be helpful later on in the treatment of these cases (cf. proof of Lemma 32).

REMARK 29

The notion of primitive terms provides the first and most basic connection of unified correspondence theory to the characterization problem of the properly displayable DLE-logics (cf. Definition 27). Indeed, it can be easily verified by direct inspection that left-primitive terms are both positive Skeleton terms and negative PIA-terms (cf. discussion after Definition 16), and right-primitive terms are both positive PIA-terms and negative Skeleton terms. In principle, not all positive PIA-terms (or negative Skeleton terms) are right-primitive, since $-\perp$ and $+\top$ are allowed to occur in their positive generation tree, while they are not allowed to occur in $+s$ for any right-primitive term $s$. Likewise, not all negative PIA-terms (or positive Skeleton terms) are left-primitive, since $+\perp$ and $-\top$ are allowed to occur in their positive generation tree, while they are not allowed to occur in $+s$ for any left-primitive term $s$.

EXAMPLE 30

Let $\mathcal{L}_{\text {DLE }}(\mathcal{F}, \mathcal{G})$ be s.t. $\mathcal{F}=\{\diamond\}$ and $\mathcal{G}=\{\rightarrow, \square\}$. Of the following Fischer Servi inequalities (cf. $[52,53])$,

$$
\diamond(q \rightarrow p) \leq \square q \rightarrow \diamond p \quad \diamond q \rightarrow \square p \leq \square(q \rightarrow p),
$$

the second one is right-primitive, whereas the first one is neither right- nor left-primitive.

Early on, in Definition 24, left-sided and right-sided structural terms were associated with formulas. In fact, it is not difficult to show, by induction on the shape of left-sided and right-sided structural terms, that the set of definite left-primitive (resp. right-primitive) formulas (cf. Definition 28) is exactly the image of the map $l$ (resp. $r$ ). The inverse maps of $l$ and $r$ are defined as follows:

DEFINITION 31 (Structures associated with definite primitive formulas)

Any definite left-primitive formula $s$ and any definite right-primitive formula $t$ is associated with structures $S=l^{-1}(s)$ and $T=r^{-1}(t)$, respectively, by the following simultaneous induction on $s$ and $t$.

$$
\begin{array}{ll}
\text { if } s=p \text { then } S:=\zeta(p) & \text { if } t=p \text { then } T:=\zeta(p) \\
\text { if } s=\top \text { then } S:=\mathrm{I} & \text { if } t=\perp \text { then } T:=\mathrm{I} \\
\text { if } s=s_{1} \wedge s_{2} \text { then } S=S_{1} ; S_{2} & \text { if } t=t_{1} \vee t_{2} \text { then } T=T_{1} ; T_{2} \\
\text { if } s=f\left(\overrightarrow{s^{\prime}} / \vec{p}, \overrightarrow{t^{\prime}} / \vec{q}\right) \text { then } S:=H\left(\vec{S}^{\prime}, \vec{T}^{\prime}\right) & \text { if } t=g\left(\overrightarrow{t^{\prime}} / \vec{p}, \overrightarrow{s^{\prime}} / \vec{q}\right) \text { then } T:=K\left(\vec{T}^{\prime}, \vec{S}^{\prime}\right)
\end{array}
$$

where $\zeta$ is an injective map from AtProp to the set of structural variables.

\section{LEMMA 32}

Every left-primitive (resp. right-primitive) inequality $s \leq t$ is semantically equivalent to a set of special structural rules in the display calculus DL.

PROOF. Assume that $s \leq t$ is right-primitive, and that both $s$ and $t$ are in conjunction normal form, i.e. $s=\bigwedge_{i \leq n} s_{i}$ and $t=\bigwedge_{j \leq k} t_{j}$, where $s_{i}$ and $t_{j}$ are definite right-primitive formulas for any $i \leq n$ and any $j \leq k$. If some variables occur in $s$ which do not occur in $t$, then item (3) of Definition 28 
guarantees that $s$, and hence the whole inequality, is uniform in these variables. Hence, as discussed in Footnote 22, the inequality $s \leq t$ can be transformed into some inequality $s^{\prime} \leq t$ in which each positive (resp. negative) occurrence of these variables has been suitably replaced by $\top$ (resp. $\perp$ ). The assumption that each term $s_{i}$ is definite right-primitive implies that each term in which the substitution has been effected is equivalent to $T$, and hence can be removed from the conjunction normal form. If the substitution has been effected on each $s_{i}$, then the inequality $s \leq t$ is equivalent to $\top \leq t$, which can be equivalently transformed into the 0 -ary rule $\mathrm{I} \vdash T$, where $T:=r^{-1}(t)$ as in Definition 31, which is immediately verified to be analytic. Assume now that all the variables which occur in $s$ occur as well in $t$. The following chain of equivalences is sound on any $\mathcal{L}_{\mathrm{DLE}}$-algebra $\mathbb{A}$ :

$$
\begin{array}{lll} 
& \forall \vec{p}[s \leq t] & \\
\text { iff } & \forall \vec{p} \forall p[p \leq s \Rightarrow p \leq t] & \\
\text { iff } & \forall \vec{p} \forall p\left[p \leq \bigwedge_{i \leq n} s_{i} \Rightarrow p \leq \bigwedge_{j \leq k} t_{j}\right] \\
\text { iff } & \forall \vec{p} \forall p\left[\&_{i \leq n} p \leq s_{i} \Rightarrow \mathbb{Q}_{j \leq k} p \leq t_{j}\right] \\
\text { iff } & \&_{j \leq k}\left(\forall \vec{p} \forall p\left[\&_{i \leq n} p \leq s_{i} \Rightarrow p \leq t_{j}\right]\right) .
\end{array}
$$

Recalling the definition of satisfaction of rules of DL on algebras (cf. Section 4.2), the chain of equivalences above proves that for every perfect $\mathcal{L}_{\mathrm{DLE}}$-algebra $\mathbb{A}$, the validity of $s \leq t$ on $\mathbb{A}$ is equivalent to the simultaneous validity on $\mathbb{A}$ of the following rules:

$$
\left(\frac{\left(X \vdash S_{i} \mid i \leq n\right)}{X \vdash T_{j}} \mid j \leq k\right)
$$

where for every $i \leq n$ and $j \leq k$, the structures $S_{i}$ and $T_{j}$ are the ones associated with $s_{i}$ and $t_{j}$, respectively, as indicated in Definition 31. With a similar argument, it can be shown that if $s \leq t$ is left-primitive and both $s$ and $t$ are in disjunction normal form (i.e. $s=\bigvee_{i \leq n} s_{i}$ and $t=\bigvee_{j \leq k} t_{j}$, where $s_{i}$ and $t_{j}$ are definite left-primitive formulas for any $i \leq n$ and any $j \leq k$ ), the validity of $s \leq t$ on $\mathbb{A}$ is equivalent to the simultaneous validity on $\mathbb{A}$ of the following rules:

$$
\left(\frac{\left(T_{j} \vdash Y \mid j \leq k\right)}{S_{i} \vdash Y} \mid i \leq n\right),
$$

where for every $i \leq n$ and $j \leq k$, the structures $S_{i}$ and $T_{j}$, are the ones associated with $s_{i}$ and $t_{j}$, respectively, as indicated in Definition 31. It remains to be shown that these rules are analytic, i.e. that they satisfy conditions $\mathrm{C}_{1}-\mathrm{C}_{7}$. Condition $\mathrm{C}_{1}$ follows from the assumption that all the variables which occur in the tail occur as well in the head. $\mathrm{C}_{5}$ imposes restrictions on the introduction of formulas, and hence is vacuously true on structural rules. Conditions $\mathrm{C}_{2}, \mathrm{C}_{6}$ and $\mathrm{C}_{7}$ are immediate. Condition $\mathrm{C}_{3}$ follows from the requirement that every proposition variable occurs only once in the head of a primitive inequality. Finally, condition $\mathrm{C}_{4}$ follows from the requirement that the formulas have the same order type on the variables they have in common.

Notice that the rules obtained from primitive inequalities in the way described above have the following special cases:

- if $s \leq t$ is a left-primitive (resp. right-primitive) inequality such that $t$ (resp. $s$ ) is definite, then the corresponding set of rules consists of unary rules;

- if $s \leq t$ is a left-primitive (resp. right-primitive) inequality $s \leq t$ such that $s$ (resp. $t$ ) is definite, then the corresponding set of rules consists of one single rule;

- if $s \leq t$ is a left-primitive (resp. right-primitive) inequality $s \leq t$ such that both $s$ and $t$ are definite, then the corresponding set of rules consists of one single unary rule. 
The other direction is also true:

LEMMA 33

Every special structural rule in the language of $\mathbf{D L}$ is semantically equivalent to some left-primitive or right-primitive inequality. ${ }^{34}$

PROOF. Let us treat the case in which the special rule is of the form

$$
\frac{\left(X \vdash S_{i} \mid i \leq n\right)}{X \vdash T} \rho,
$$

where $X$ does not occur in any $S_{i}$ nor in $T$. Let $l(X)=p$ and let $\vec{q}$ be the variables that appear in $r\left(S_{i}\right)$ and $r(T)$. As discussed in Section 4.2, the semantic validity of the rule above can be expressed as follows:

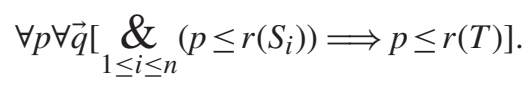

The fact that $X$ does not occur in any $S_{i}$ nor in $T$ implies that $p$ does not occur in $r\left(S_{i}\right)$ and $r(T)$. Then the above quasi-inequality can be equivalently rewritten as follows:

$$
\forall \vec{q}\left[\bigwedge_{i \leq n} r\left(S_{i}\right) \leq r(T)\right]
$$

The inequality between brackets is right-primitive: indeed, similarly to what has been discussed above Definition 31 it is not difficult to show that $\bigwedge_{i \leq n} r\left(S_{i}\right)$ and $r(T)$ are right-primitive terms. Moreover, the assumption that $\rho$ is special implies that it is analytic, and hence $\rho$ satisfies conditions $\mathrm{C}_{1}-\mathrm{C}_{7}$. Condition $\mathrm{C}_{3}$ guarantees that $r(T)$ is scattered and hence item (1). of Definition 28 is satisfied. Condition $\mathrm{C}_{1}$ guarantees Condition $\mathrm{C}_{4}$ guarantees that $\bigwedge_{i<n} r\left(S_{i}\right)$ and $r(T)$ are uniform w.r.t. the same order type and hence items (2). and (3). are satisfied.

\section{EXAMPLE 34}

Let $\mathcal{F}=\{\diamond\}$ and $\mathcal{G}=\{\rightarrow, \square\}$. The logical connectives of the display calculi DL and DL* associated with the basic $\mathcal{L}_{\mathrm{DLE}}(\mathcal{F}, \mathcal{G})$-logic can be represented synoptically as follows:

\begin{tabular}{|r|c|c|c|c|c|c|c|c|c|c|}
\hline Structural symbols & \multicolumn{2}{|c|}{$\mathrm{I}$} & \multicolumn{2}{|c|}{$;$} & \multicolumn{2}{|c|}{$>$} & \multicolumn{2}{|c|}{$\circ$} & \multicolumn{2}{|c|}{$\bullet$} \\
\hline Operational symbols & $\top$ & $\perp$ & $\wedge$ & $\vee$ & $(>-)$ & $\rightarrow$ & $\diamond$ & $\square$ & $(\bullet)$ & $(\mathbf{\square})$ \\
\hline
\end{tabular}

Below we illustrate schematically how to apply the procedure above to the Fischer Servi inequality $\diamond q \rightarrow \square p \leq \square(q \rightarrow p)$, which is right-primitive (cf. Example 30):

$$
\diamond q \rightarrow \square p \leq \square(q \rightarrow p) \quad \rightsquigarrow \quad \frac{x \vdash \diamond q \rightarrow \square p}{x \vdash \square(q \rightarrow p)} \rightsquigarrow \frac{X \vdash \circ Z>\circ Y}{X \vdash \circ(Z>Y)} .
$$

\footnotetext{
${ }^{34}$ Notice that translating rules as axioms of the original DLE-language instead of as inequalities (as done e.g. in [10, Theorem 4.5]) is possible only if the basic logic has an implication-type connective with modus ponens. In the present logical setting this is not possible in general.
} 


\subsection{Order-theoretic properties of primitive inequalities}

The following lemma identifies the most important order-theoretic feature induced by the syntactic shape of primitive inequalities. Notice that, by definition, any scattered term $s$ is monotone, hence $s$ can be associated with an order type on its variables, which is denoted $\varepsilon_{s}$. In these cases, we will sometimes write $s(\vec{p}, \vec{q})$ with the convention that $\varepsilon_{S}(p)=1$ for any $p$ in $\vec{p}$, and $\varepsilon_{s}(q)=\partial$ for any $q$ in $\vec{q}$. Also, in what follows we will find it convenient to represent an array $\vec{s}=\left(s_{1}, \ldots, s_{n}\right)$ as $\left(\overrightarrow{s_{-i}}, s_{i}\right)$ for $1 \leq i \leq n$, where $\overrightarrow{s_{-i}}:=\left(s_{1}, \ldots, s_{i-1}, s_{i+1}, \ldots, s_{n}\right)$. Finally, we write e.g. $s(\vec{u} / \vec{p})$ to indicate that the arrays $\vec{u}$ and $\vec{p}$ have the same length $n$ and that, for each $1 \leq i \leq n$, the formula $u_{i}$ has been uniformly substituted in $s$ for the variable $p_{i}$.

\section{LEMMA 35}

For every language $\mathcal{L}_{\text {DLE }}$, any definite and scattered left-primitive (resp. right-primitive) $\mathcal{L}_{\text {DLE-term }}$ $s$ and any $\mathcal{L}_{\text {DLE-algebra }} \mathbb{A}$, the term function $s^{\mathbb{A}}: \mathbb{A}^{\varepsilon_{s}} \rightarrow \mathbb{A}$ is a (dual) operator, and if $\mathbb{A}$ is perfect, then $s^{\mathbb{A}}: \mathbb{A}^{\varepsilon_{s}} \rightarrow \mathbb{A}$ is a complete (dual) operator. ${ }^{35}$

ProOF. By induction on the complexity of $s$. If $s$ is a constant or a proposition variable, the verification of the statement is immediate. Let $s=f(\vec{u}, \vec{v})=f(\vec{u} / \vec{p}, \vec{v} / \vec{q})$. The assumptions that $s$ is definite, leftprimitive and scattered and those on the order type of $s$ imply that each $u$ in $\vec{u}$ is definite, leftprimitive, and scattered, and each $v$ in $\vec{v}$ is definite, right-primitive, and scattered. Hence, by induction hypothesis, the term function $u^{\mathbb{A}}: \mathbb{A}^{\varepsilon_{s}} \rightarrow \mathbb{A}$ is an operator for each $u$, and $v^{\mathbb{A}}: \mathbb{A}^{\varepsilon_{s}^{\partial}} \rightarrow \mathbb{A}$ is a dual operator for each $v$. Let $r$ be a variable occurring in $s$, and assume that the only occurrence of $r$ belongs to a subterm $u_{i}$. If $\varepsilon_{s}(r)=1$, then $\varepsilon_{u_{i}}(r)=1$, and hence

$$
\begin{aligned}
f\left(\vec{u}_{-i}, u_{i}\left[\left(\bigvee_{j \in I} \phi_{j}\right) / r\right], \vec{v}\right) & =f\left(\vec{u}_{-i},\left(\bigvee_{j \in I} u_{i}\left[\phi_{j} / r\right]\right), \vec{v}\right) \quad \text { (induction hypothesis) } \\
& =\bigvee_{j \in I} f\left(\vec{u}_{-i}, u_{i}\left[\phi_{j} / r\right], \vec{v}\right) .
\end{aligned}
$$

If $\varepsilon_{S}(r)=\partial$, then $\varepsilon_{u_{i}}(r)=\partial$, hence

$$
\begin{aligned}
f\left(\vec{u}_{-i}, u_{i}\left[\left(\bigwedge_{j \in I} \phi_{j}\right) / r\right], \vec{v}\right) & =f\left(\vec{u}_{-i},\left(\bigvee_{j \in I} u_{i}\left[\phi_{j} / r\right]\right), \vec{v}\right) \quad \text { (induction hypothesis) } \\
& =\bigvee_{j \in I} f\left(\vec{u}_{-i}, u_{i}\left[\phi_{j} / r\right], \vec{v}\right) .
\end{aligned}
$$

The remaining cases can be proven with similar arguments.

\section{COROLlary 36}

The following rules are sound and invertible in perfect DLEs, and derivable in ALBA for any definite scattered left-primitive term $s(\vec{p}, \vec{q})$ and definite scattered right-primitive term $t(\vec{p}, \vec{q})$ :

$$
(\operatorname{Approx}(s)) \frac{\mathbf{j} \leq s(\vec{p}, \vec{q})}{\mathbf{j} \leq s(\overrightarrow{\mathbf{i}}, \overrightarrow{\mathbf{m}}) \quad \overrightarrow{\mathbf{i}} \leq \vec{p} \quad \vec{q} \leq \overrightarrow{\mathbf{m}}} \quad \frac{t(\vec{p}, \vec{q}) \leq \mathbf{m}}{t(\overrightarrow{\mathbf{n}}, \overrightarrow{\mathbf{i}}) \leq \mathbf{m} \quad \vec{p} \leq \overrightarrow{\mathbf{m}} \quad \overrightarrow{\mathbf{i}} \leq \vec{q}}(\operatorname{Approx}(t))
$$

ProOF. The first part of the statement is an immediate consequence of Lemma 35. The second part can be straightforwardly shown by induction on $s$ and $t$. The details of the proof are omitted

\footnotetext{
${ }^{35} \mathrm{An}$ operation on a lattice $\mathbb{A}$ is an operator (resp. a dual operator) if it preserves finite joins (resp. meets) in each coordinate. Notice that this condition includes the preservation of the empty join $\perp$ (resp. the empty meet $T$ ). An operation on a complete lattice is a complete operator (resp. a complete dual operator) if it preserves all joins (resp. meets) in each coordinate.
} 
PROPOSITION 37

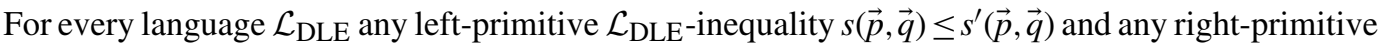

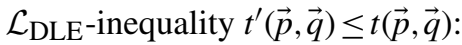

(1) If $s(\vec{p}, \vec{q})$ is definite, then the following are equivalent for every perfect DLE $\mathbb{A}$ :

(a) $\mathbb{A} \models s(\vec{p}, \vec{q}) \leq s^{\prime}(\vec{p}, \vec{q})$;

(b) $\mathbb{A} \models s(\overrightarrow{\mathbf{i}}, \overrightarrow{\mathbf{m}}) \leq s^{\prime}(\overrightarrow{\mathbf{i}}, \overrightarrow{\mathbf{m}})$.

(2) If $t(\vec{p}, \vec{q})$ is definite, then the following are equivalent for every perfect DLE $\mathbb{A}$ :

(a) $\mathbb{A} \models t^{\prime}(\vec{p}, \vec{q}) \leq t(\vec{p}, \vec{q})$;

(b) $\mathbb{A} \models t^{\prime}(\overrightarrow{\mathbf{m}}, \overrightarrow{\mathbf{i}}) \leq t(\overrightarrow{\mathbf{m}}, \overrightarrow{\mathbf{i}})$.

PROOF. We only prove 1, the proof of item (2) being order dual. By the assumptions and Corollary 36 , the following chain of equivalences can be obtained via an ALBA reduction and hence is sound on perfect DLEs:

$$
\begin{array}{lll} 
& \forall \vec{p} \forall \vec{q}\left[s(\vec{p}, \vec{q}) \leq s^{\prime}(\vec{p}, \vec{q})\right] & \\
\text { iff } & \forall \vec{p} \forall \vec{q} \forall \mathbf{j}\left[\mathbf{j} \leq s(\vec{p}, \vec{q}) \Rightarrow \mathbf{j} \leq s^{\prime}(\vec{p}, \vec{q})\right] \\
\text { iff } & \forall \vec{p} \forall \vec{q} \forall \mathbf{j} \forall \mathbf{i} \forall \mathbf{m}\left[(\overrightarrow{\mathbf{i}} \leq \vec{p} \& \vec{q} \leq \overrightarrow{\mathbf{m}} \& \mathbf{j} \leq s(\overrightarrow{\mathbf{i}}, \overrightarrow{\mathbf{m}})) \Rightarrow \mathbf{j} \leq s^{\prime}(\vec{p}, \vec{q})\right] & (\text { Approx }(s)) \\
\text { iff } & \forall \mathbf{j} \forall \overrightarrow{\mathbf{i}} \forall \overrightarrow{\mathbf{m}}\left[\mathbf{j} \leq s(\overrightarrow{\mathbf{i}}, \overrightarrow{\mathbf{m}}) \Rightarrow \mathbf{j} \leq s^{\prime}(\overrightarrow{\mathbf{i}}, \overrightarrow{\mathbf{m}})\right] & \text { (Ackermann, } s, s^{\prime} \text { same order type) } \\
\text { iff } & \forall \overrightarrow{\mathbf{i}} \forall \overrightarrow{\mathbf{m}}\left[s(\overrightarrow{\mathbf{i}}, \overrightarrow{\mathbf{m}}) \leq s^{\prime}(\overrightarrow{\mathbf{i}}, \overrightarrow{\mathbf{m}})\right] &
\end{array}
$$

\section{REMARK 38}

Proposition 37 can be straightforwardly generalized to primitive inequalities the heads of which are not definite. For any such inequality, the preprocessing stage of ALBA produces a set of definite primitive inequalities with definite heads, to each of which Proposition 37 can then be applied separately. Notice that the preprocessing does not affect the order type of the occurring variables. Then, one can reverse the preprocessing steps and transform the set of pure definite primitive inequalities into a substitution instance of the input primitive inequality in which proposition variables have been suitably substituted for nominals and conominals.

EXAMPLE 39

Let us illustrate the reduction strategy of the proposition above by applying it to the right-primitive Fischer Servi inequality discussed in Examples 30 and 34 (cf. [42, Lemma 27]).

$$
\begin{array}{ll} 
& \forall q \forall p[\diamond q \rightarrow \square p \leq \square(q \rightarrow p)] \\
\text { iff } & \forall q \forall p \forall \mathbf{i} \forall \mathbf{m}[(\mathbf{i} \leq \diamond q \rightarrow \square p \& \square(q \rightarrow p) \leq \mathbf{m}) \Rightarrow \mathbf{i} \leq \mathbf{m}] \\
\text { iff } & \forall q \forall p \forall \mathbf{i} \forall \mathbf{m} \forall \mathbf{n}[(\mathbf{i} \leq \diamond q \rightarrow \square p \& \square(q \rightarrow \mathbf{n}) \leq \mathbf{m} \& p \leq \mathbf{n}) \Rightarrow \mathbf{i} \leq \mathbf{m}] \\
\text { iff } & \forall q \forall \mathbf{i} \forall \mathbf{m} \forall \mathbf{n}[(\mathbf{i} \leq \diamond q \rightarrow \square \mathbf{n} \& \square(q \rightarrow \mathbf{n}) \leq \mathbf{m}) \Rightarrow \mathbf{i} \leq \mathbf{m}] \\
\text { iff } & \forall q \forall \mathbf{i} \forall \mathbf{m} \forall \mathbf{n} \forall \mathbf{j}[(\mathbf{i} \leq \diamond q \rightarrow \square \mathbf{n} \& \square(\mathbf{j} \rightarrow \mathbf{n}) \leq \mathbf{m} \& \mathbf{j} \leq q) \Rightarrow \mathbf{i} \leq \mathbf{m}] \\
\text { iff } & \forall \mathbf{i} \forall \mathbf{m} \forall \mathbf{n} \forall \mathbf{j}[(\mathbf{i} \leq \diamond \mathbf{j} \rightarrow \square \mathbf{n} \& \square(\mathbf{j} \rightarrow \mathbf{n}) \leq \mathbf{m}) \Rightarrow \mathbf{i} \leq \mathbf{m}] \\
\text { iff } & \forall \mathbf{i} \forall \mathbf{n} \forall \mathbf{j}[\mathbf{i} \leq \diamond \mathbf{j} \rightarrow \square \mathbf{n} \Rightarrow \forall \mathbf{m}[\square(\mathbf{j} \rightarrow \mathbf{n}) \leq \mathbf{m} \Rightarrow \mathbf{i} \leq \mathbf{m}]] \\
\text { iff } & \forall \mathbf{i} \forall \mathbf{n} \forall \mathbf{j}[\mathbf{i} \leq \diamond \mathbf{j} \rightarrow \square \mathbf{n} \Rightarrow \mathbf{i} \leq \square(\mathbf{j} \rightarrow \mathbf{n})] \\
\text { iff } & \forall \mathbf{n} \forall \mathbf{j}[\diamond \mathbf{j} \rightarrow \square \mathbf{n} \leq \square(\mathbf{j} \rightarrow \mathbf{n})] .
\end{array}
$$




\subsection{Special rules via ALBA: main strategy}

Before moving on to the next section, in the present subsection we take stock of the facts we have collected so far, and spell out their role in the context of the method we will apply in the following section. This method is to extend the class of primitive inequalities in any given language $\mathcal{L}_{\text {DLE }}$ to classes of inequalities each element of which can be equivalently (and effectively) transformed into (a set of) special structural rules, hence giving rise to specially displayable DLE-logics (cf. Definition 27). This method is based on the simple but crucial observation that the languages of the display calculi DL, DL* and DL* (cf. Definition 21 and Footnote 32) are built using the same set of structural connectives. For each language $\mathcal{L}_{\mathrm{DLE}}$, we are going to identify classes of non-primitive $\mathcal{L}_{\text {DLE}}$-inequalities which can be equivalently and effectively transformed into (conjunctions of) primitive inequalities in the expanded language $\mathcal{L}_{\text {DLE }}^{*}$ (cf. Section 3.2). By Lemma 32 applied to $\mathcal{L}_{\text {DLE }}^{*}$, each primitive $\mathcal{L}_{\text {DLE }}^{*}$-inequality can then be equivalently transformed into a set of special structural rules in the language of $\mathbf{D L}^{*}$, which, as observed above, coincides with the structural language of DL.

Proposition 37 provides a key step in the procedure to equivalently transform input $\mathcal{L}_{\mathrm{DLE}^{-}}$ inequalities into primitive $\mathcal{L}_{\mathrm{DLE}}^{*}$-inequalities. Indeed, it guarantees that each definite primitive $\mathcal{L}_{\text {DLE }}^{*}$-inequality is equivalent to a 'substitution instance of itself' in which all the nominals and conominals have been uniformly substituted for proposition variables, as illustrated by the right-hand vertical equivalence in the diagram below:

$$
\begin{array}{ll}
\mathbb{A} \models s(\vec{p}, \vec{q}) \leq s^{\prime}(\vec{p}, \vec{q}) & \mathbb{A} \models \&\left\{s_{i}^{*}(\vec{p}, \vec{q}) \leq s_{i}^{\prime *}(\vec{p}, \vec{q}) \mid i \in I\right\} \\
\mathbb{1} \text { Theorems } 19 \text { and } 17 & \text { 步 Proposition } 37 \\
\mathbb{A} \models \&\left\{s_{i}^{*}(\overrightarrow{\mathbf{i}}, \overrightarrow{\mathbf{m}}) \leq s_{i}^{\prime *}(\overrightarrow{\mathbf{i}}, \overrightarrow{\mathbf{m}}) \mid i \in I\right\} & \mathbb{A} \models \&\left\{s_{i}^{*}(\overrightarrow{\mathbf{i}}, \overrightarrow{\mathbf{m}}) \leq s_{i}^{\prime *}(\overrightarrow{\mathbf{i}}, \overrightarrow{\mathbf{m}}) \mid i \in I\right\}
\end{array}
$$

Our task in the following section will be to perform ALBA-reductions aimed at equivalently transforming $\mathcal{L}_{\mathrm{DLE}}$-inequalities into sets of definite pure primitive $\mathcal{L}_{\mathrm{DLE}}^{*}$-inequalities, so as to provide the left-hand side leg of the diagram above.

\section{Extending the class of primitive inequalities}

In the present section, we introduce a hierarchy of classes of $\mathcal{L}_{\mathrm{DLE}}$-inequalities which properly extend primitive inequalities, and which can be equivalently (and effectively) transformed into sets of special structural rules (cf. Definition 6), via progressively more complex ALBA-reduction strategies. The classes of inequalities treated in the present section are all proper subclasses of the class of analytic inductive inequalities (cf. Definition 55), which is the most general, and which, in Section 7, will be also shown to capture analytic rules modulo equivalence. However, the procedure described in Section 7 does not deliver special rules in the restricted sense of Definition 6 in general, whereas the finer analysis provided in the present section is guaranteed to yield special rules in this restricted sense (cf. Remark 7) in each instance in which it is applicable. Thus, unlike the general procedure, the procedure described in the present section provides a direct and fully mechanized way ${ }^{36}$ to obtain

\footnotetext{
${ }^{36}$ In Section 8, we will show that in fact, all DLE-logics axiomatized by analytic inductive inequalities are specially displayable. However, the general procedure, derived from the results in Sections 7 and 8, to extract special rules from analytic inductive inequalities is indirect, as it consists of more than one back-and-forth toggle between inequalities and rules.
} 
specially displayable DLE-logics (cf. Definition 27). Section 7 is independent from the present section, hence the reader is not constrained to read the present section before the next. Finally, the present article is intended for two very different readerships; in this respect, the present section, which is the richest in examples of the whole article, can be useful to the reader who wishes to become familiar with ALBA reductions.

Throughout the present section, we adopt the convention that $f(\vec{p}, \vec{q})$ and $g(\vec{p}, \vec{q})$ are s.t. $\varepsilon_{f}(p)=$ $\varepsilon_{g}(p)=1$ for every $p \in \vec{p}$ and $\varepsilon_{f}(q)=\varepsilon_{g}(q)=\partial$ for every $q \in \vec{q}$. For any sequence of formulas $\vec{\psi}=$ $\left(\psi_{1}, \ldots, \psi_{n}\right)$ and any $1 \leq i \leq n$, we let $\overrightarrow{\psi_{-i}}:=\left(\psi_{1}, \ldots, \psi_{i-1}, \psi_{i+1}, \ldots, \psi_{n}\right)$.

\subsection{Type 2: allowing multiple occurrences of critical variables}

By definition, each proposition letter in the head of a primitive inequality is required to occur at most once (i.e. the head of primitive inequalities is required to be scattered). The present subsection is aimed at showing that this condition can be relaxed.

DEFINITION 40 (Quasi-primitive inequalities)

An inequality $s_{1} \leq s_{2}$ is quasi left-primitive (resp. quasi right-primitive) if both $s_{1}$ and $s_{2}$ are monotone (w.r.t. some order type $\varepsilon_{s_{i}}$ ) and left-primitive (resp. right-primitive) formulas, and moreover $s_{1}$ and $s_{2}$ have the same order type relative to the variables they have in common.

The definition above differs from Definition 28 in that the requirement that the head be scattered is dropped.

\section{REMARK 41}

In what follows, we are going to provide an effective procedure to equivalently transform quasiprimitive inequalities into pure primitive inequalities. We will restrict our focus to quasi-primitive inequalities with definite head (cf. Proposition 44). Indeed, during the pre-processing stage of the execution of ALBA, each quasi-primitive inequality with non-definite head can be equivalently transformed into (the conjunction of) a set of quasi-primitive inequalities with definite head, on each of which the procedure described below can be effected in parallel. Thus, this restriction is without loss of generality.

\section{DEFINITION 42}

For every left-primitive (resp. right-primitive) formula $s(\vec{p}, \vec{q})$, a scattered transform of $s$ is a scattered left-primitive (resp. right-primitive) term $s^{*}\left(\overrightarrow{p^{\prime}}, \overrightarrow{q^{\prime}}\right)$ for which there exists a substitution $\sigma: \operatorname{AtProp}\left(s^{*}\right) \rightarrow \operatorname{AtProp}(s)$ such that $s(\vec{p}, \vec{q})=\sigma\left(s^{*}\left(\overrightarrow{p^{\prime}}, \overrightarrow{q^{\prime}}\right)\right)$.

Clearly, we can always assume without loss of generality that $s(\vec{p}, \vec{q})$ and $s^{*}\left(\vec{p}^{\prime}, \vec{q}^{\prime}\right)$ share no variables. In particular, in the following lemma, we will find it useful to consider scattered transforms which are pure, i.e. of the form $s^{*}(\overrightarrow{\mathbf{i}}, \overrightarrow{\mathbf{m}})$ or $s^{*}(\overrightarrow{\mathbf{m}}, \overrightarrow{\mathbf{i}})$, and such that their associated substitution $\sigma$ maps nominals and conominals to proposition variables in a suitable way according to their polarity. This can always be done without loss of generality.

LEMMA 43

The following rules are sound and invertible in perfect DLEs and are derivable in ALBA:

(1) For any definite quasi left-primitive term $s(\vec{p}, \vec{q})$,

$$
\underset{\mathbf{j} \leq s^{*}(\overrightarrow{\mathbf{i}}, \overrightarrow{\mathbf{m}}) \quad \overrightarrow{\mathbf{j} \leq s(\vec{p}, \vec{q})}}{\overrightarrow{\bigvee \sigma^{-1}[p]} \leq \vec{p} \quad \vec{q} \leq \overrightarrow{\bigwedge \sigma^{-1}[q]}}\left(\operatorname{Approx}_{\sigma}(s)\right)
$$


where, for every $p$ in $\vec{p}$ and every $q$ in $\vec{q}$, every variable in $\sigma^{-1}[p]$ is a (fresh) nominal, and every variable in $\sigma^{-1}[q]$ is a (fresh) conominal, and $s^{*}$ is the scattered transform of $s$ induced by $\sigma$.

(2) For any definite quasi right-primitive term $t(\vec{p}, \vec{q})$ :

$$
\underset{t^{*}(\overrightarrow{\mathbf{n}}, \overrightarrow{\mathbf{i}}) \leq \mathbf{m} \quad \vec{p} \leq \overrightarrow{\bigwedge \sigma^{-1}[p]} \quad \overrightarrow{\vee \sigma^{-1}[q]} \leq \vec{q}}{\longrightarrow}\left(\operatorname{Approx}_{\sigma}(t)\right)
$$

where, for every $p$ in $\vec{p}$ and every $q$ in $\vec{q}$, every variable in $\sigma^{-1}[p]$ is a (fresh) conominal, and every variable in $\sigma^{-1}[q]$ is a (fresh) nominal, and $t^{*}$ is the scattered transform of $t$ induced by $\sigma$.

Proof. We only prove item (1), item (2) being order-dual.

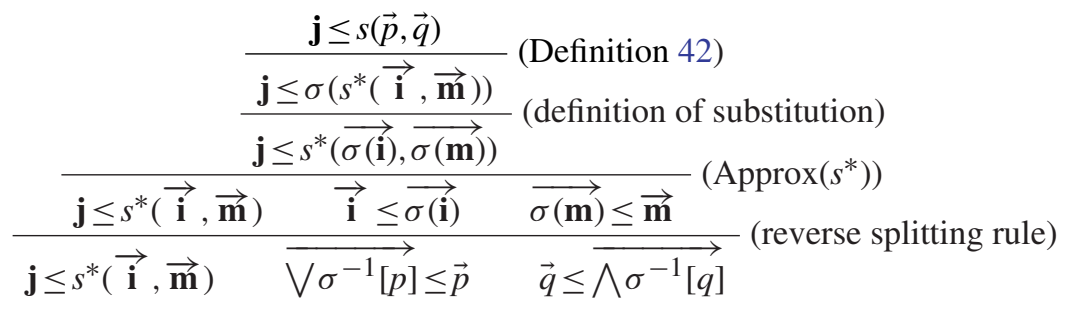

The following proposition and its proof provide an effective procedure to equivalently transform quasi-primitive inequalities with definite head into pure primitive inequalities.

\section{PROPOSITION 44}

For every quasi left-primitive inequality $s(\vec{p}, \vec{q}) \leq s^{\prime}(\vec{p}, \vec{q})$ such that $s$ is definite and every quasi right-primitive inequality $t^{\prime}(\vec{p}, \vec{q}) \leq t(\vec{p}, \vec{q})$ such that $t$ is definite:

(1) The following are equivalent for every perfect $\mathcal{L}_{\mathrm{DLE}}$ algebra $\mathbb{A}$ :

(a) $\mathbb{A} \models s(\vec{p}, \vec{q}) \leq s^{\prime}(\vec{p}, \vec{q})$;

(b) $\mathbb{A} \models s^{*}(\overrightarrow{\mathbf{i}}, \overrightarrow{\mathbf{m}}) \leq s^{\prime}\left(\overrightarrow{\bigvee \sigma^{-1}[p]}, \overrightarrow{\wedge \sigma^{-1}[q]}\right)$,

where $s^{*}$ is a pure scattered transform of $s$ witnessed by a map $\sigma: \operatorname{Prop}\left(s^{*}\right) \rightarrow \operatorname{Prop}(s)$ such that, for every $p$ in $\vec{p}$ and every $q$ in $\vec{q}$, every variable in $\sigma^{-1}[p]$ is a nominal and every variable in $\sigma^{-1}[q]$ is a conominal.

(2) The following are equivalent for every perfect $\mathcal{L}_{\text {DLE }}$ algebra $\mathbb{A}$ :

(a) $\mathbb{A} \models t^{\prime}(\vec{p}, \vec{q}) \leq t(\vec{p}, \vec{q})$;

(b) $\mathbb{A} \models t^{\prime}\left(\overrightarrow{\bigwedge \sigma^{-1}[p]}, \overrightarrow{\bigvee \sigma^{-1}[q]}\right) \leq t^{*}(\overrightarrow{\mathbf{m}}, \overrightarrow{\mathbf{i}})$,

where $t^{*}$ is a pure scattered transform of $t$ witnessed by a map $\sigma: \operatorname{Prop}\left(t^{*}\right) \rightarrow \operatorname{Prop}(t)$ such that, for every $p$ in $\vec{p}$ and every $q$ in $\vec{q}$, every variable in $\sigma^{-1}[p]$ is a conominal and every variable in $\sigma^{-1}[q]$ is a nominal. 
ProOF. We only prove item (1), item (2) being order-dual. The assumptions and Lemma 43 guarantee that the following ALBA reduction is sound:

$$
\begin{aligned}
& \forall \vec{p} \forall \vec{q}\left[s(\vec{p}, \vec{q}) \leq s^{\prime}(\vec{p}, \vec{q})\right] \\
& \text { iff } \quad \forall \vec{p} \forall \vec{q} \forall \mathbf{j}\left[\mathbf{j} \leq s(\vec{p}, \vec{q}) \Rightarrow \mathbf{j} \leq s^{\prime}(\vec{p}, \vec{q})\right] \\
& \text { iff } \forall \vec{p} \forall \vec{q} \forall \mathbf{j} \forall \overrightarrow{\mathbf{i}} \forall \overrightarrow{\mathbf{m}}\left[\left(\mathbf{j} \leq s^{*}(\overrightarrow{\mathbf{i}}, \overrightarrow{\mathbf{m}}) \& \overrightarrow{\sigma^{-1}[p]} \leq \vec{p} \& \vec{q} \leq \overrightarrow{\bigwedge \sigma^{-1}[q]}\right) \Rightarrow \mathbf{j} \leq t(\vec{p}, \vec{q})\right]\left(\operatorname{Approx}_{\sigma}(\mathrm{s})\right) \\
& \text { iff } \quad \forall \mathbf{j} \forall \overrightarrow{\mathbf{i}} \forall \overrightarrow{\mathbf{m}}\left[\mathbf{j} \leq s^{*}(\overrightarrow{\mathbf{i}}, \overrightarrow{\mathbf{m}}) \Rightarrow \mathbf{j} \leq s^{\prime}\left(\overrightarrow{\bigvee \sigma^{-1}[p]}, \overrightarrow{\wedge \sigma^{-1}[q]}\right)\right] \text { (Ackermann, } s, s^{\prime} \text { same order type) } \\
& \text { iff } \forall \overrightarrow{\mathbf{i}} \forall \overrightarrow{\mathbf{m}}\left[s^{*}(\overrightarrow{\mathbf{i}}, \overrightarrow{\mathbf{m}}) \leq s^{\prime}\left(\overrightarrow{\bigvee \sigma^{-1}[p]}, \overrightarrow{\wedge \sigma^{-1}[q]}\right)\right] \text {. }
\end{aligned}
$$

\subsubsection{A concrete instantiation of the method}

Let $\mathcal{F}=\{\cdot, \diamond\}$ and $\mathcal{G}=\varnothing$, where $\cdot$ is binary and of order type $(1,1)$. The inequality $\diamond \diamond p \cdot \diamond p \leq \diamond p$ is quasi left-primitive and definite, and fails to be left-primitive because its head (the term on the left-hand side) is not scattered. First, we run ALBA on this inequality, so as to equivalently transform it into a pure non-definite left-primitive inequality as follows:

$$
\begin{array}{ll} 
& \forall p[\diamond \diamond p \cdot \diamond p \leq \diamond p] \\
\text { iff } & \forall p \forall \mathbf{j} \forall \mathbf{m}[(\mathbf{j} \leq \diamond \diamond p \cdot \diamond p \& \diamond p \leq \mathbf{m}) \Rightarrow \mathbf{j} \leq \mathbf{m}] \\
\text { iff } & \forall p \forall \mathbf{j} \forall \mathbf{m} \forall \mathbf{i}[(\mathbf{j} \leq \diamond \diamond \mathbf{i} \cdot \diamond p \& \mathbf{i} \leq p \& \diamond p \leq \mathbf{m}) \Rightarrow \mathbf{j} \leq \mathbf{m}] \\
\text { iff } & \forall p \forall \mathbf{j} \forall \mathbf{m} \forall \mathbf{i} \forall \mathbf{h}[(\mathbf{j} \leq \diamond \diamond \mathbf{i} \cdot \diamond \mathbf{h} \& \mathbf{i} \leq p \& \mathbf{h} \leq p \& \diamond p \leq \mathbf{m}) \Rightarrow \mathbf{j} \leq \mathbf{m}] \\
\text { iff } & \forall p \forall \mathbf{j} \forall \mathbf{m} \forall \mathbf{i} \forall \mathbf{h}[(\mathbf{j} \leq \diamond \diamond \mathbf{i} \cdot \diamond \mathbf{h} \& \mathbf{i} \vee \mathbf{h} \leq p \& \diamond p \leq \mathbf{m}) \Rightarrow \mathbf{j} \leq \mathbf{m}] \\
\text { iff } & \forall \mathbf{j} \forall \mathbf{m} \forall \mathbf{i} \forall \mathbf{h}[(\mathbf{j} \leq \diamond \diamond \mathbf{i} \cdot \diamond \mathbf{h} \& \diamond(\mathbf{i} \vee \mathbf{h}) \leq \mathbf{m}) \Rightarrow \mathbf{j} \leq \mathbf{m}] \\
\text { iff } & \forall \mathbf{j} \forall \mathbf{i} \forall \mathbf{h}[\mathbf{j} \leq \diamond \diamond \mathbf{i} \cdot \diamond \mathbf{h} \Rightarrow \forall \mathbf{m}[\diamond(\mathbf{i} \vee \mathbf{h}) \leq \mathbf{m} \Rightarrow \mathbf{j} \leq \mathbf{m}]] \\
\text { iff } & \forall \mathbf{j} \forall \mathbf{i} \forall \mathbf{h}[\mathbf{j} \leq \diamond \diamond \mathbf{i} \cdot \diamond \mathbf{h} \Rightarrow \mathbf{j} \leq \diamond(\mathbf{i} \vee \mathbf{h})] \\
\text { iff } & \forall \mathbf{i} \forall \mathbf{h}[\diamond \diamond \mathbf{i} \cdot \diamond \mathbf{h} \leq \diamond(\mathbf{i} \vee \mathbf{h})]
\end{array}
$$

\begin{tabular}{|c|c|c|c|c|c|}
\hline Structural symbols & $\circ$ & - & $\odot$ & oll & $/ / \odot$ \\
\hline Operational symbols & $\diamond$ & $(\mathbf{\square})$ & . & $()$. & (/.) \\
\hline
\end{tabular}

By Proposition 37, the pure left-primitive inequality $\diamond \diamond \mathbf{i} \cdot \diamond \mathbf{h} \leq \diamond(\mathbf{i} \vee \mathbf{h})$ is equivalent on perfect $\mathcal{L}_{\text {DLE }}(\mathcal{F}, \mathcal{G})$-algebras to the left-primitive inequality $\diamond \diamond p_{1} \cdot \diamond p_{2} \leq \diamond\left(p_{1} \vee p_{2}\right)$, which, via ALBAdistribution rule, is equivalent to the following inequality in disjunction normal form:

$$
\diamond \diamond p_{1} \cdot \diamond p_{2} \leq \diamond p_{1} \vee \diamond p_{2}
$$

If we specify the non-lattice fragment of the language of the associated calculus DL as follows:

then, applying the procedure indicated in the proof of Lemma 32, the inequality above can be transformed into a structural rule in the language above as follows:

$$
\diamond \diamond p_{1} \cdot \diamond p_{2} \leq \diamond p_{1} \vee \diamond p_{2} \rightsquigarrow \frac{\diamond p_{1} \vdash z \quad \diamond p_{2} \vdash z}{\diamond \diamond p_{1} \cdot \diamond p_{2} \vdash z} \rightsquigarrow \frac{\circ X \vdash Z \quad \circ Y \vdash Z}{\circ \circ X \odot \circ Y \vdash Z} .
$$

\subsubsection{Monotone terms in quasi-primitive inequalities}

The head of primitive inequalities is scattered, hence monotone (w.r.t. some order type). In defining quasi-primitive inequalities, we have dropped the former requirement but kept the latter. Before 
moving on, let us illustrate why by means of an example. Let $\mathcal{F}=\{\cdot, \diamond, \triangleleft\}$ and $\mathcal{G}=\varnothing$, where $\cdot$ is binary and of order type $(1,1)$, and $\triangleleft$ is unary and of order type (2). The inequality $p \cdot \triangleleft p \leq \diamond p$ is not quasi-primitive, since its head $p \cdot \triangleleft p$ is not monotone. Actually, this inequality behaves like a primitive inequality, in that Proposition 37 can be generalized to cover such an inequality; indeed

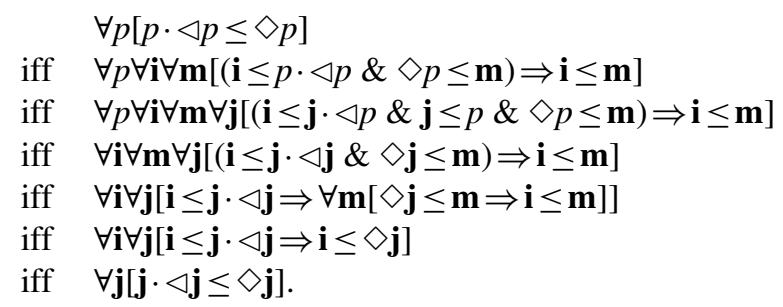

However, this is not good news. Indeed, this reduction does not help to solve the main problem of this inequality, namely the fact that if we apply the procedure described in the proof of Lemma 32 to this inequality, we obtain a rule which violates condition $\mathrm{C}_{4}$ (position-alikeness of parameters).

\subsection{Type 3: allowing PIA-subterms}

In Sections 5.2 and 6.1, we have generalized Kracht's notion of primitive inequalities, first by making this notion apply uniformly to any $\mathcal{L}_{\mathrm{DLE}}$-signature, and then by dropping the requirement that the heads of inequalities be scattered. Moreover, we have identified the main order-theoretic features induced by the syntactic shape of definite scattered primitive formulas, and, thanks to this identification, we have started to see ALBA at work on primitive and quasi-primitive inequalities. However, so far we have not discussed why ALBA was guaranteed to succeed on any primitive or quasi-primitive inequality in the first place. More in general, we have not yet made use of the second tool of unified correspondence theory: the possibility of identifying Sahlqvist and inductive type of inequalities in any $\mathcal{L}_{\mathrm{DLE}}$-signature.

So let us start the present subsection by analysing (quasi-)primitive inequalities as inductive inequalities (cf. Definition 16). Indeed, it can be easily verified by direct inspection that all primitive inequalities are a very special subclass of inductive $\mathcal{L}_{\text {DLE-inequalities. Specifically, as observed }}$ earlier (cf. Remark 29), all non-leaf nodes in the generation tree $+s$ (resp. $-s$ ) of a (quasi) leftprimitive (resp. right-primitive) formula $s$ are Skeleton nodes. This guarantees that, if $+s$ is also monotone w.r.t. some order type $\varepsilon_{s}$, then all the variables at the leaves of such a generation tree (which are $\varepsilon_{s}$-critical) can be solved for, and moreover (together with the condition on the order type in Definition 40), that an ALBA reduction on a (quasi-)primitive inequality is guaranteed to reach Ackermann shape using only approximation and splitting rules after the preprocessing stage.

A natural question arising at this point is whether or not all inductive inequalities can be transformed via ALBA into (conjunctions of) pure primitive inequalities, as outlined in Section 5.3. We can already answer this question in the negative, as the following example shows. Let $\mathcal{F}=\{\diamond\}$ and $\mathcal{G}=\{\square\}$, and consider the inequality $\diamond p \leq \diamond \square p$, which is Sahlqvist for the order type (1) and 'McKinsey-type' for the order type ( $\partial$ ), and is neither left-primitive nor right-primitive. There is only one successful reduction strategy for ALBA, which consists in solving for the positive occurrence of $p$ as follows:

$$
\begin{array}{ll} 
& \forall p[\diamond p \leq \diamond \square p] \\
\text { iff } & \forall p \forall \mathbf{i} \forall \mathbf{m}[(\mathbf{i} \leq \diamond p \& \diamond \square p \leq \mathbf{m}) \Rightarrow \mathbf{i} \leq \mathbf{m}] \\
\text { iff } & \forall p \forall \mathbf{i} \forall \mathbf{m} \forall \mathbf{j}[(\mathbf{i} \leq \diamond \mathbf{j} \& \mathbf{j} \leq p \& \diamond \square p \leq \mathbf{m}) \Rightarrow \mathbf{i} \leq \mathbf{m}]
\end{array}
$$




$$
\begin{array}{ll}
\text { iff } & \forall \mathbf{i} \forall \mathbf{m} \forall \mathbf{j}[(\mathbf{i} \leq \diamond \mathbf{j} \& \diamond \square \mathbf{j} \leq \mathbf{m}) \Rightarrow \mathbf{i} \leq \mathbf{m}] \\
\text { iff } & \forall \mathbf{i} \forall \mathbf{j}[\mathbf{i} \leq \diamond \mathbf{j} \Rightarrow \forall \mathbf{m}[\diamond \square \mathbf{j} \leq \mathbf{m} \Rightarrow \mathbf{i} \leq \mathbf{m}]] \\
\text { iff } & \forall \mathbf{i} \forall \mathbf{j}[\mathbf{i} \leq \diamond \mathbf{j} \Rightarrow \mathbf{i} \leq \diamond \square \mathbf{j}] \\
\text { iff } & \forall \mathbf{j}[\diamond \mathbf{j} \leq \diamond \square \mathbf{j}] .
\end{array}
$$

Clearly, this reduction fails to improve the situation, since it leaves the troublemaking side $\diamond \square p$ untouched. In contrast to this example, consider the inequality $\diamond \square p \leq \diamond p$, which is again neither left-primitive nor right-primitive, but is Sahlqvist for both order types (1) and (2). Solving for the troublemaking side we obtain:

$$
\begin{array}{ll} 
& \forall p[\diamond \square p \leq \diamond p] \\
\text { iff } & \forall p \forall \mathbf{i} \forall \mathbf{m}[(\mathbf{i} \leq \diamond \square p \& \diamond p \leq \mathbf{m}) \Rightarrow \mathbf{i} \leq \mathbf{m}] \\
\text { iff } & \forall p \forall \mathbf{i} \forall \mathbf{m} \forall \mathbf{j}[(\mathbf{i} \leq \diamond \mathbf{j} \& \mathbf{j} \leq \square p \& \diamond p \leq \mathbf{m}) \Rightarrow \mathbf{i} \leq \mathbf{m}] \\
\text { iff } & \forall p \forall \mathbf{i} \forall \mathbf{m} \forall \mathbf{j}[(\mathbf{i} \leq \diamond \mathbf{j} \& \diamond \mathbf{j} \leq p \& \diamond p \leq \mathbf{m}) \Rightarrow \mathbf{i} \leq \mathbf{m}] \\
\text { iff } & \forall \mathbf{i} \forall \mathbf{m} \forall \mathbf{j}[(\mathbf{i} \leq \diamond \mathbf{j} \& \diamond \diamond \mathbf{j} \leq \mathbf{m}) \Rightarrow \mathbf{i} \leq \mathbf{m}] \\
\text { iff } & \forall \mathbf{i} \forall \mathbf{j}[\mathbf{i} \leq \diamond \mathbf{j} \Rightarrow \forall \mathbf{m}[\diamond \bullet \mathbf{j} \leq \mathbf{m} \Rightarrow \mathbf{i} \leq \mathbf{m}]] \\
\text { iff } & \forall \mathbf{i} \forall \mathbf{j}[\mathbf{i} \leq \diamond \mathbf{j} \Rightarrow \mathbf{i} \leq \diamond \diamond \mathbf{j}] \\
\text { iff } & \forall \mathbf{j}[\diamond \mathbf{j} \leq \diamond \diamond \mathbf{j}],
\end{array}
$$

from which the usual steps (Proposition 37 and Lemma 32) yield the rule

$$
\frac{\circ \bullet X \vdash Y}{\circ X \vdash Y}
$$

These ideas motivate the following:

DEFINITION 45 (Very restricted analytic inductive inequalities)

For any order type $\varepsilon$ and any irreflexive and transitive relation $\Omega$ on the variables $p_{1}, \ldots p_{n}$, the signed generation tree $* s(* \in\{+,-\})$ of a term $s\left(p_{1}, \ldots p_{n}\right)$ is restricted analytic $(\Omega, \varepsilon)$-inductive if

(1) $* s$ is $(\Omega, \varepsilon)$-inductive (cf. Definition 16);

(2) every branch of $* s$ is good (cf. Definition 15);

(3) every maximal $\varepsilon^{\partial}$-uniform subtree of $* s$ occurs as an immediate subtree of an SRR node of some $\varepsilon$-critical branch of $* s$;

An inequality $s \leq t$ is very restricted left-analytic $(\Omega, \varepsilon)$-inductive (resp. very restricted right-analytic $(\Omega, \varepsilon)$-inductive $)$ if

(1) $+s$ (resp. $-t$ ) (which we refer to as the head of the inequality) is restricted analytic $(\Omega, \varepsilon)$ inductive;

(2) $-t($ resp. $+s)$ is $\varepsilon^{\partial}$-uniform, and

(3) $t$ is left-primitive (resp. $s$ is right-primitive) (cf. Definition 28).

An inequality $s \leq t$ is very restricted analytic inductive if it is very restricted (right-analytic or leftanalytic) $(\Omega, \varepsilon)$-inductive for some $\Omega$ and $\varepsilon$.

\section{REMARK 46}

The syntactic shape specified in the definition above can be intuitively understood with the help of the following picture, which illustrates the 'left-analytic' case: 


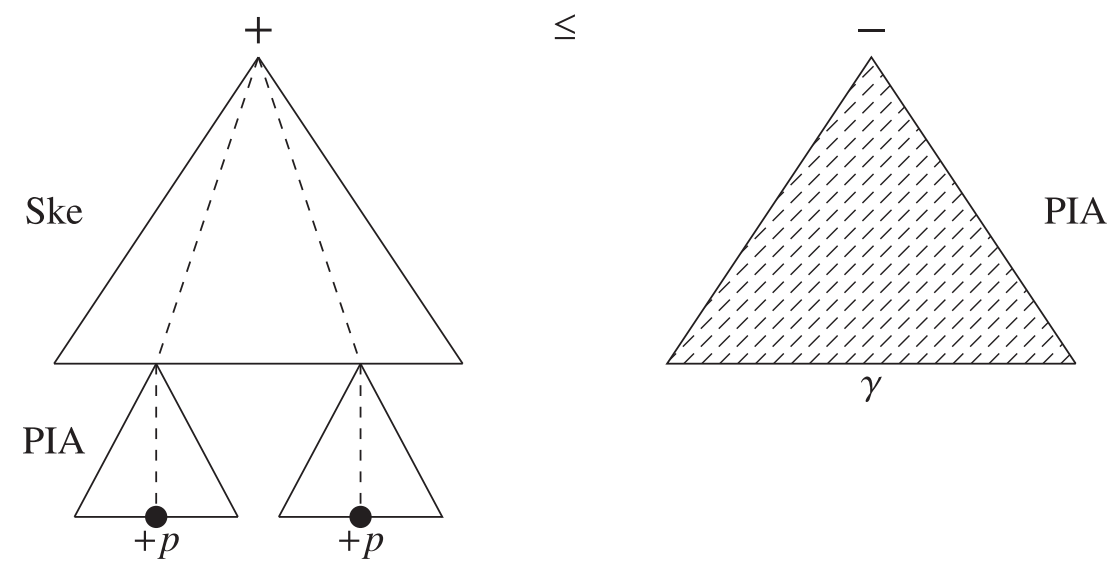

As the picture shows, this syntactic shape requires that each $\varepsilon$-critical occurrence is a leaf of the head of the inequality. Moreover, the definition of restricted analytic $(\Omega, \varepsilon)$-inductive signed generation tree implies that every maximal PIA-subtree contains at least one (but possibly more) $\varepsilon$-critical variable occurrence. Further, the requirement that every branch be good implies that every maximal $\varepsilon^{\partial}$-subtree $\gamma$ of every PIA-structure consists also exclusively of PIA-nodes. Moreover, the requirement that these subtrees be attached to their main PIA-subtree by means of an SRR-node lying on a critical branch guarantees that these subtrees will be incorporated in the minimal valuation subtree of the critical occurrence at the leaf of that critical branch.

Finally, exhaustively applying the distribution rules (a')-(c') described in Remark 18 to any restricted analytic inductive term produces a restricted analytic inductive term, every maximal PIA-subterm of which is definite (cf. Footnote 29) and contains exactly one $\varepsilon$-critical variable occurrence.

EXAMPLE 47

Let $\mathcal{F}=\varnothing$ and $\mathcal{G}=\{\rightarrow\}$, with $\rightarrow$ binary and of order type $(\partial, 1)$. As observed in [18], the Frege inequality

$$
p \rightarrow(q \rightarrow r) \leq(p \rightarrow q) \rightarrow(p \rightarrow r)
$$

is not Sahlqvist for any order type, but is $(\Omega, \varepsilon)$-inductive, e.g. for $r<\Omega p<\Omega q$ and $\varepsilon(p, q, r)=(1,1, \partial)$, and is also very restricted right-analytic $(\Omega, \varepsilon)$-inductive for the same $\Omega$ and $\varepsilon$, as can be seen from the signed generation trees below:
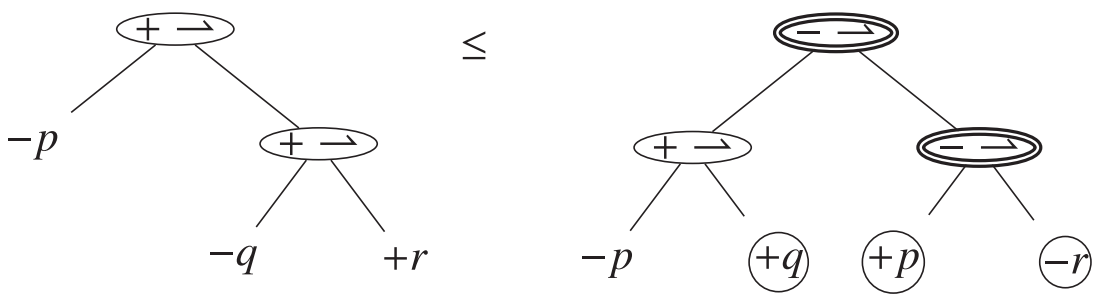

In the picture above, the circled variable occurrences are the $\varepsilon$-critical ones, the doubly circled nodes are the Skeleton ones and the single-circle ones are PIA.

Below, we introduce an auxiliary definition which is a simplified version of [14, Definition 5.1] and is aimed at effectively calculating the residuals of definite positive and negative PIA formulas 
(cf. discussion after Definition 16 and Footnote 29) w.r.t. a given variable occurrence $x$. The intended meaning of notation such as $\phi(! x, \bar{z})$ is that the variable $x$ occurs exactly once in the formula $\phi$.

DEFINITION 48

For every definite positive PIA $\mathcal{L}_{\text {DLE }}$-formula $\phi=\phi(! x, \bar{z})$, and any definite negative PIA $\mathcal{L}_{D L E^{-}}$ formula $\psi=\psi(! x, \bar{z})$ such that $x$ occurs in them exactly once, the $\mathcal{L}_{\mathrm{DLE}}^{+}$-formulas $\operatorname{LA}(\phi)(u, \bar{z})$ and $\operatorname{RA}(\psi)(u, \bar{z})$ (for $u \in \operatorname{Var}-(x \cup \bar{z})$ ) are defined by simultaneous recursion as follows:

$$
\begin{aligned}
& \operatorname{LA}(x)=u \\
& \operatorname{LA}(\square \phi(x, \bar{z})) \quad=\quad \operatorname{LA}(\phi)(\bullet u, \bar{z}) ; \\
& \operatorname{LA}(\psi(\bar{z}) \rightarrow \phi(x, \bar{z}))=\operatorname{LA}(\phi)(u \wedge \psi(\bar{z}), \bar{z}) ; \\
& \operatorname{LA}\left(\phi_{1}(\bar{z}) \vee \phi_{2}(x, \bar{z})\right)=\operatorname{LA}\left(\phi_{2}\right)\left(u-\phi_{1}(\bar{z}), \bar{z}\right) ; \\
& \mathrm{LA}(\psi(x, \bar{z}) \rightarrow \phi(\bar{z}))=\operatorname{RA}(\psi)(u \rightarrow \phi(\bar{z}), \bar{z}) ; \\
& \mathrm{LA}\left(g\left(\overrightarrow{\phi_{-j}(\vec{z})}, \phi_{j}(x, \bar{z}), \overrightarrow{\psi(\vec{z})}\right)\right)=\operatorname{LA}\left(\phi_{j}\right)\left(g_{j}^{b}\left(\overrightarrow{\phi_{-j}(\vec{z})}, u, \overrightarrow{\psi(\vec{z})}\right), \bar{z}\right) ; \\
& \operatorname{LA}\left(g\left(\overrightarrow{\phi(\bar{z})}, \overrightarrow{\psi_{-j}(\vec{z})}, \psi_{j}(x, \bar{z})\right)\right)=\operatorname{RA}\left(\psi_{j}\right)\left(g_{j}^{b}\left(\overrightarrow{\phi(\bar{z})}, \overrightarrow{\psi_{-j}(\bar{z})}, u\right), \bar{z}\right) ; \\
& \mathrm{RA}(x)=u \\
& \operatorname{RA}(\diamond \psi(x, \bar{z}))=\operatorname{RA}(\psi)(\square u, \bar{z}) \\
& \operatorname{RA}(\psi(x, \bar{z})-\phi(\bar{z}))=\operatorname{RA}(\psi)(\phi(\bar{z}) \vee u, \bar{z}) ; \\
& \operatorname{RA}\left(\psi_{1}(\bar{z}) \wedge \psi_{2}(x, \bar{z})\right)=\operatorname{RA}\left(\psi_{2}\right)\left(\psi_{1}(\bar{z}) \rightarrow u, \bar{z}\right) ; \\
& \operatorname{RA}(\psi(\bar{z})-\phi(x, \bar{z}))=\operatorname{LA}(\phi)(\psi(\bar{z})-u, \bar{z}) ; \\
& \operatorname{RA}\left(f\left(\overrightarrow{\psi_{-j}(\bar{z})}, \psi_{j}(x, \bar{z}), \overrightarrow{\phi(\vec{z})}\right)\right)=\operatorname{RA}\left(\psi_{j}\right)\left(f_{j}^{\sharp}\left(\overrightarrow{\psi_{-j}(\bar{z})}, u, \overrightarrow{\phi(\bar{z})}\right), \bar{z}\right) ; \\
& \operatorname{RA}\left(f\left(\overrightarrow{\psi(\vec{z})}, \overrightarrow{\phi_{-j}(\vec{z})}, \phi_{j}(x, \bar{z})\right)\right)=\operatorname{LA}\left(\phi_{j}\right)\left(f_{j}^{\sharp}\left(\overrightarrow{\psi(\vec{z})}, \overrightarrow{\phi_{-j}(\vec{z})}, u\right), \bar{z}\right) .
\end{aligned}
$$

LEMMA 49

For all definite positive PIA $\mathcal{L}_{\text {DLE-formulas }} \phi_{1}(! x, \bar{z}), \phi_{2}(! x, \bar{z})$, and all definite negative PIA $\mathcal{L}_{\mathrm{DLE}^{-}}$ formulas $\psi_{1}(! x, \bar{z}), \psi_{2}(! x, \bar{z})$ such that the variable $x$ occurs in them exactly once:

(1) If $+x \prec+\phi_{1}$, then the following rule is derivable in ALBA:

$$
\left(\operatorname{LA}\left(\phi_{1}\right)\right) \frac{\chi \leq \phi_{1}(x, \bar{z})}{\operatorname{LA}\left(\phi_{1}\right)(\chi / u, \bar{z}) \leq x}
$$

and moreover, $\operatorname{LA}\left(\phi_{1}\right)(u, \bar{z})$ is a definite negative PIA $\mathcal{L}_{\mathrm{DLE}}^{*}$-formula.

(2) If $-x \prec+\phi_{2}$, then the following rule is derivable in ALBA:

$$
\left(\operatorname{LA}\left(\phi_{2}\right)\right) \frac{\chi \leq \phi_{2}(x, \bar{z})}{x \leq \operatorname{LA}\left(\phi_{2}\right)(\chi / u, \bar{z})}
$$

and moreover, $\operatorname{LA}\left(\phi_{2}\right)(u, \bar{z})$ is a definite positive PIA $\mathcal{L}_{\mathrm{DLE}}^{*}$-formula.

(3) If $+x \prec+\psi_{1}$, then the following rule is derivable in ALBA:

$$
\left(\operatorname{RA}\left(\psi_{1}\right)\right) \frac{\psi_{1}(x, \bar{z}) \leq \chi}{x \leq \operatorname{RA}\left(\psi_{1}\right)(\chi / u, \bar{z})}
$$

and moreover, $\operatorname{RA}\left(\psi_{1}\right)(u, \bar{z})$ is a definite positive PIA $\mathcal{L}_{\mathrm{DLE}}^{*}$-formula.

(4) If $-x \prec+\psi_{2}$, then the following rule is derivable in ALBA:

$$
\left(\operatorname{RA}\left(\psi_{2}\right)\right) \frac{\psi_{2}(x, \bar{z}) \leq \chi}{\operatorname{RA}\left(\psi_{2}\right)(\chi / u, \bar{z}) \leq x}
$$

and moreover, $\operatorname{RA}\left(\psi_{2}\right)(u, \bar{z})$ is a definite negative PIA $\mathcal{L}_{\text {DLE }}^{*}$-formula. 
PROOF. By simultaneous induction on the shapes of $\phi_{1}, \phi_{2}, \psi_{1}$ and $\psi_{2}$. The case in which they coincide with $x$ immediately follows from the definitions involved. As to the inductive step, we only illustrate the case in which $\phi_{1}$ is of the form $g\left(\overrightarrow{\phi(\bar{z})}, \overrightarrow{\psi_{-j}(\bar{z})}, \psi_{2, j}(x, \bar{z})\right)$ for some array $\overrightarrow{\phi(\bar{z})}$ of formulas which are positive PIA, some array $\overrightarrow{\psi-j(\vec{z})}$ of formulas which are negative PIA, and some negative PIA formula $\psi_{2, j}(x, \bar{z})$ such that $-x \prec+\psi_{2, j}$. Then, by induction hypothesis, the following rule is derivable in ALBA for every formula $\chi^{\prime}$ :

$$
\left(\operatorname{RA}\left(\psi_{2, j}\right)\right) \frac{\psi_{2, j}(x, \bar{z}) \leq \chi^{\prime}}{\operatorname{RA}\left(\psi_{2, j}\right)\left(\chi^{\prime} / u^{\prime}, \bar{z}\right) \leq x}
$$

Moreover, by definition,

$$
\operatorname{LA}\left(g\left(\overrightarrow{\phi(\bar{z})}, \overrightarrow{\psi_{-j}(\bar{z})}, \psi_{2, j}(x, \bar{z})\right)\right)=\operatorname{RA}\left(\psi_{2, j}\right)\left(g_{j}^{b}\left(\overrightarrow{\phi(\bar{z})}, \overrightarrow{\psi_{-j}(\bar{z})}, u\right) / u^{\prime}, \bar{z}\right)
$$

Hence, we can show that $\operatorname{RA}\left(\phi_{1}\right)$ is a derivable ALBA-rule as follows: for every formula $\chi$,

$$
\begin{gathered}
\frac{\chi \leq g\left(\overrightarrow{\phi(\vec{z})}, \overrightarrow{\psi_{-j}(\vec{z})}, \psi_{2, j}(x, \bar{z})\right)}{\psi_{2, j}(x, \bar{z}) \leq g_{j}^{b}\left(\overrightarrow{\phi(\vec{z})}, \overrightarrow{\psi_{-j}(\vec{z})}, \chi / u\right)} \text { (Residuation) } \\
\frac{\operatorname{RA}\left(\psi_{2, j}\right)\left(g_{j}^{b}\left(\overrightarrow{\phi(\vec{z})}, \overrightarrow{\psi_{-j}(\bar{z})}, \chi / u\right) / u^{\prime}, \bar{z}\right) \leq x}{\left.\operatorname{LA}\left(g(\overrightarrow{\phi(\bar{z})}), \overrightarrow{\psi_{-j}(\vec{z})}, \psi_{2, j}(x, \bar{z})\right)\right)(\chi / u, \bar{z}) \leq x}(\text { Identity }(6.1))
\end{gathered}
$$

To see that $\operatorname{LA}\left(\phi_{1}\right)(u, \bar{z})$ is a definite negative PIA-formula, one needs to show that every positive (resp. negative) node in $+\mathrm{LA}\left(\phi_{1}\right)(u, \bar{z})$ is labelled with a connective from $\mathcal{F}^{*}$ (resp. $\mathcal{G}^{*}$ ). This follows from the identity (6.1), the second part of the induction hypothesis (stating that $\operatorname{RA}\left(\psi_{2, j}\right)\left(u^{\prime}, \bar{z}\right)$ is definite negative PIA), the fact that $\operatorname{RA}\left(\psi_{2, j}\right)\left(u^{\prime}, \bar{z}\right)$ is negative in $u^{\prime}$, the fact that $g_{j}^{b} \in \mathcal{G}^{*}$ (and its corresponding node in $+\operatorname{LA}\left(\phi_{1}\right)(u, \bar{z})$ is signed - , as we have just remarked), the fact that the order type of $g_{j}^{b}$ is the same as the order type of $g$, the fact that every formula in $\overrightarrow{\phi(\vec{z})}$ is positive PIA, and for each $\phi$ in $\overrightarrow{\phi(\bar{z})}$,

$$
-\phi \prec-g_{j}^{b}\left(\overrightarrow{\phi(\vec{z})}, \overrightarrow{\psi_{-j}(\vec{z})}, \chi / u\right) \prec+\operatorname{LA}\left(\phi_{1}\right)(u, \bar{z}),
$$

and finally, the fact that every formula in $\overrightarrow{\psi_{-j}(\bar{z})}$ is negative PIA, and for each $\psi$ in $\overrightarrow{\psi_{-j}(\bar{z})}$,

$$
+\psi \prec-g_{j}^{b}(\overrightarrow{\phi(\bar{z})}, \overrightarrow{\psi-j(\bar{z})}, \chi / u) \prec+\operatorname{LA}\left(\phi_{1}\right)(u, \bar{z}) .
$$

\section{THEOREM 50}

Every very restricted left-analytic (resp. right-analytic) inductive $\mathcal{L}_{\mathrm{DLE}}$-inequality can be equivalently transformed, via an ALBA-reduction, into a set of pure left-primitive (resp. right-primitive) $\mathcal{L}_{\mathrm{DLE}^{-}}^{*}$ inequalities.

PROOF. We only consider the case of the inequality $s \leq t$ being very restricted left-analytic $(\Omega, \varepsilon)$ inductive, since the proof of the right-analytic case is dual. By assumption, $t$ is a negative PIA formula (cf. paragraph after Definition 16, page 20). Observe preliminarily that we can assume w.l.o.g. that there are no occurrences of $+\perp$ and $-\top$ in $+t$. Indeed, modulo exhaustive application of distribution rules, $t$ can be equivalently written as the disjunction of definite negative PIA terms $t_{i}$. 
If $+\perp$ or $-\top$ occurred in $+t_{i}$ for some $i$, the exhaustive application of the rules which identify each $+f^{\prime}\left(\phi_{1}, \ldots, \perp^{\varepsilon_{f^{\prime}}}(i), \ldots, \phi_{n_{f^{\prime}}}\right)$ with $+\perp$ for every $f^{\prime} \in \mathcal{F} \cup\{\wedge\}$ and $-g^{\prime}\left(\phi_{1}, \ldots, \top^{\varepsilon_{g^{\prime}}}(i), \ldots, \phi_{n_{g^{\prime}}}\right)$ with $-\top$ for every $g^{\prime} \in \mathcal{G} \cup\{\vee\}$ would identify $t_{i}$ with $\perp$. Hence, the offending subterm can be removed from the disjunction. Hence (cf. Remark 29), we can assume w.l.o.g. that $t$ is left-primitive.

By assumption, $s:=\xi(\vec{\phi} / \vec{x}, \vec{\psi} / \vec{y})$, where $\xi(\overrightarrow{!}, \vec{y})$ is a positive Skeleton formula-cf. page 20, paragraph after Definition 16-which is scattered, monotone in $\vec{x}$ and antitone in $\vec{y}$. Moreover, the formulas in $\vec{\phi}$ are positive PIA, and the formulas in $\vec{\psi}$ are negative PIA. Modulo exhaustive application of distribution and splitting rules of the standard ALBA preprocessing, ${ }^{37}$ we can assume w.l.o.g. that the scattered positive Skeleton formula $\xi$ is also definite. Modulo exhaustive application of the additional rules which identify $+f^{\prime}\left(\phi_{1}, \ldots, \perp^{\varepsilon_{f^{\prime}}}(i), \ldots, \phi_{n_{f^{\prime}}}\right)$ with $+\perp$ for every $f^{\prime} \in \mathcal{F} \cup\{\wedge\}$ and $-g^{\prime}\left(\phi_{1}, \ldots, \top^{\varepsilon} g^{\prime}(i), \ldots, \phi_{n_{g^{\prime}}}\right)$ with $-\top$ for every $g^{\prime} \in \mathcal{G} \cup\{\vee\}$, which would reduce $s \leq t$ to a tautology, we can assume w.l.o.g. that there are no occurrences of $+\perp$ and $-\top$ in $+\xi$. Hence (cf. Remark 29), we can assume w.l.o.g. that $\xi$ is scattered, definite and left-primitive. Therefore, the derived rule $\operatorname{Approx}(\xi)$ (cf. Corollary 36) is applicable, which justifies the last equivalence in the following chain:

$$
\begin{array}{ll} 
& \forall \vec{p}[\xi(\vec{\phi} / \vec{x}, \vec{\psi} / \vec{y}) \leq t(\vec{p})] \\
\text { iff } & \forall \vec{p} \forall \mathbf{j} \forall \mathbf{n}[(\mathbf{j} \leq \xi(\vec{\phi} / \vec{x}, \vec{\psi} / \vec{y}) \& t(\vec{p}) \leq \mathbf{n}) \Rightarrow \mathbf{j} \leq \mathbf{n}] \\
\text { iff } & \forall \vec{p} \forall \mathbf{j} \forall \mathbf{n} \forall \overrightarrow{\mathbf{i}} \forall \overrightarrow{\mathbf{m}}[(\mathbf{j} \leq \xi(\overrightarrow{\mathbf{i}} / \vec{x}, \overrightarrow{\mathbf{m}} / \vec{y}) \& \overrightarrow{\mathbf{i}} \leq \vec{\phi} \& \vec{\psi} \leq \overrightarrow{\mathbf{m}} \& t(\vec{p}) \leq \mathbf{n}) \Rightarrow \mathbf{j} \leq \mathbf{n}] . \quad(\operatorname{Approx}(\xi))
\end{array}
$$

By assumption, in each inequality $\overrightarrow{\mathbf{i}} \leq \vec{\phi}$ and $\vec{\psi} \leq \overrightarrow{\mathbf{m}}$ there is at least one $\varepsilon$-critical variable occurrence. Modulo exhaustive application of distribution rules (a')-(c') of Remark 18 and splitting rules, we can assume w.l.o.g. that each $\phi$ in $\vec{\phi}$ (resp. $\psi$ in $\vec{\psi}$ ) is a definite positive (resp. negative) PIAformula, which has exactly one $\varepsilon$-critical variable occurrence. That is, if $\overrightarrow{p_{1}}$ and $\overrightarrow{p_{2}}$, respectively, denote the subarrays of $\vec{p}$ such that $\varepsilon\left(p_{1}\right)=1$ for each $p_{1}$ in $\overrightarrow{p_{1}}$ and $\varepsilon\left(p_{2}\right)=\partial$ for each $p_{2}$ in $\overrightarrow{p_{2}}$, then each $\phi$ in $\vec{\phi}$ is either of the form $\phi_{1}\left(p_{1} / ! x, \overrightarrow{p^{\prime}} / \bar{z}\right)$ with $+x \prec+\phi_{1}$, or of the form $\phi_{2}\left(p_{2} / ! x, \vec{p}^{\prime} / \vec{z}\right)$ with $-x \prec+\phi_{2}$. Similarly, each $\psi$ in $\vec{\psi}$ is either of the form $\psi_{1}\left(p_{2} / ! x, \vec{p}^{\prime} / \bar{z}\right)$ with $+x \prec-\psi_{1}$, or of the form $\psi_{2}\left(p_{1} / ! x, \vec{p}^{\prime} / \bar{z}\right)$ with $-x \prec-\psi_{2}$. Recall that each $\phi$ in $\vec{\phi}$ is definite positive PIA and each $\psi$ in $\vec{\psi}$ is definite negative PIA. Hence, we can assume w.l.o.g. that there are no occurrences of $-\perp$ and $+\top$ in $+\phi$. Indeed, otherwise, the exhaustive application of the additional rules which identify $-f^{\prime}\left(\phi_{1}, \ldots, \perp^{\varepsilon_{f^{\prime}}}(i), \ldots, \phi_{n_{f^{\prime}}}\right)$ with $-\perp$ for every $f^{\prime} \in \mathcal{F} \cup\{\wedge\}$ and $+g^{\prime}\left(\phi_{1}, \ldots, \top^{\varepsilon_{g^{\prime}}}(i), \ldots, \phi_{n_{g^{\prime}}}\right)$ with $+\top$ for every $g^{\prime} \in \mathcal{G} \cup\{\vee\}$, would reduce all offending inequalities to tautological inequalities of the form $\mathbf{i} \leq \top$ which can then be removed. Likewise, we can assume w.l.o.g. that there are no occurrences of $+\perp$ and $-\top$ in $+\psi$. This shows (cf. Remark 29) that we can assume w.l.o.g. that each $\phi$ in $\vec{\phi}$ (resp. $\psi$ in $\vec{\psi}$ ) is a right-primitive (resp. left-primitive) term. Moreover, by Lemma 49, the suitable derived adjunction rule among $\operatorname{LA}\left(\phi_{1}\right), \operatorname{LA}\left(\phi_{2}\right), \operatorname{RA}\left(\psi_{1}\right), \operatorname{RA}\left(\psi_{2}\right)$ is applicable to each formula, yielding:

$$
\text { iff } \begin{aligned}
& \forall \overrightarrow{p_{1}} \forall \overrightarrow{p_{2}} \forall \mathbf{j} \forall \mathbf{n} \forall \overrightarrow{\mathbf{i}} \forall \overrightarrow{\mathbf{m}}\left[\left(\mathbf{j} \leq \xi(\overrightarrow{\mathbf{i}} / \vec{x}, \overrightarrow{\mathbf{m}} / \vec{y}) \& \overrightarrow{\mathbf{i}} \leq \vec{\phi} \& \vec{\psi} \leq \overrightarrow{\mathbf{m}} \& t\left(\overrightarrow{p_{1}}, \overrightarrow{p_{2}}\right) \leq \mathbf{n}\right) \Rightarrow \mathbf{j} \leq \mathbf{n}\right] \\
& \quad \overrightarrow{p_{1}} \forall \overrightarrow{p_{2}} \forall \mathbf{j} \forall \mathbf{n} \forall \overrightarrow{\mathbf{i}} \forall \overrightarrow{\mathbf{m}}\left[\left(\mathbf{j} \leq \xi(\overrightarrow{\mathbf{i}} / \vec{x}, \overrightarrow{\mathbf{m}} / \vec{y}) \& \overrightarrow{\operatorname{LA}\left(\phi_{1}\right)(\mathbf{i} / u)} \leq \overrightarrow{p_{1}} \& \overrightarrow{p_{2}} \leq \overrightarrow{\operatorname{LA}\left(\phi_{2}\right)(\mathbf{i} / u)} \&\right.\right. \\
& \left.\left.\operatorname{RA}\left(\psi_{2}\right)(\mathbf{m} / u) \leq \vec{p}_{1} \& \overrightarrow{p_{2}} \leq \overrightarrow{\operatorname{RA}\left(\psi_{1}\right)(\mathbf{m} / u)} \& t\left(\overrightarrow{p_{1}}, \overrightarrow{p_{2}}\right) \leq \mathbf{n}\right) \Rightarrow \mathbf{j} \leq \mathbf{n}\right] .
\end{aligned}
$$

The assumptions made above imply that $t\left(\vec{p}_{1}, \overrightarrow{p_{2}}\right)$ is monotone in each variable in $\vec{p}_{1}$ and antitone in each variable $\overrightarrow{p_{2}}$. Hence, the quasi-inequality above is simultaneously in Ackermann shape w.r.t. all

\footnotetext{
${ }^{37}$ The applications of splitting rules at this stage give rise to a set of inequalities, each of which can be treated separately. In the remainder of the proof, we focus on one of them.
} 
1412 Unified correspondence as a proof-theoretic tool

variables. ${ }^{38}$ Applying the Ackermann rule repeatedly in the order indicated by $\Omega$ yields the following pure quasi-inequality:

$$
\forall \mathbf{j} \forall \mathbf{n} \forall \overrightarrow{\mathbf{i}} \forall \overrightarrow{\mathbf{m}}\left[\left(\mathbf{j} \leq \xi(\overrightarrow{\mathbf{i}} / \vec{x}, \overrightarrow{\mathbf{m}} / \vec{y}) \& t\left(\vec{P}_{1} / \vec{p}_{1}, \vec{P}_{2} / \vec{p}_{2}\right) \leq \mathbf{n}\right) \Rightarrow \mathbf{j} \leq \mathbf{n}\right],
$$

where $P_{1}$ and $P_{2}$ denote the pure $\mathcal{L}_{\mathrm{DLE}}^{*}$-terms obtained by applying the Ackermann substitution. For instance, for every $\Omega$-minimal $p_{1}$ in $\overrightarrow{p_{1}}$,

$$
P_{1}:=\bigvee_{i} \operatorname{LA}\left(\phi_{1}^{(i)}\right)(\mathbf{i} / u) \vee \bigvee_{j} \operatorname{RA}\left(\psi_{2}^{(j)}\right)(\mathbf{m} / u)
$$

and for every $\Omega$-minimal $p_{2}$ in $\overrightarrow{p_{2}}$,

$$
P_{2}:=\bigwedge_{i} \operatorname{LA}\left(\phi_{2}^{(i)}\right)(\mathbf{i} / u) \wedge \bigwedge_{j} \operatorname{RA}\left(\psi_{1}^{(j)}\right)(\mathbf{m} / u)
$$

In the clauses above, the indexes $i$ and $j$ count the number of critical occurrences of the given variable $p_{1}$ (resp. $p_{2}$ ) in PIA-subterms of type $\phi_{1}$ and $\psi_{2}$ (resp. $\phi_{2}$ and $\psi_{1}$ ). The pure quasi-inequality above can be equivalently transformed into one pure inequality as follows:

$$
\begin{array}{ll} 
& \forall \mathbf{j} \forall \mathbf{n} \forall \overrightarrow{\mathbf{i}} \forall \overrightarrow{\mathbf{m}}\left[\left(\mathbf{j} \leq \xi(\overrightarrow{\mathbf{i}} / \vec{x}, \overrightarrow{\mathbf{m}} / \vec{y}) \& t\left(\vec{P}_{1} / \vec{p}_{1}, \vec{P}_{2} / \overrightarrow{p_{2}}\right) \leq \mathbf{n}\right) \Rightarrow \mathbf{j} \leq \mathbf{n}\right] \\
\text { iff } & \forall \mathbf{j} \forall \overrightarrow{\mathbf{i}} \forall \overrightarrow{\mathbf{m}}\left[\left(\mathbf{j} \leq \xi(\overrightarrow{\mathbf{i}} / \vec{x}, \overrightarrow{\mathbf{m}} / \vec{y}) \Rightarrow \forall \mathbf{n}\left[t\left(\vec{P}_{1} / \vec{p}_{1}, \vec{P}_{2} / \vec{p}_{2}\right) \leq \mathbf{n} \Rightarrow \mathbf{j} \leq \mathbf{n}\right]\right]\right. \\
\text { iff } & \forall \mathbf{j} \forall \overrightarrow{\mathbf{i}} \forall \overrightarrow{\mathbf{m}}\left[\mathbf{j} \leq \xi(\overrightarrow{\mathbf{i}} / \vec{x}, \overrightarrow{\mathbf{m}} / \vec{y}) \Rightarrow \mathbf{j} \leq t\left(\vec{P}_{1} / \vec{p}_{1}, \vec{P}_{2} / \vec{p}_{2}\right)\right] \\
\text { iff } & \forall \overrightarrow{\mathbf{i}} \forall \overrightarrow{\mathbf{m}}\left[\xi(\overrightarrow{\mathbf{i}} / \vec{x}, \overrightarrow{\mathbf{m}} / \vec{y}) \leq t\left(\vec{P}_{1} / \vec{p}_{1}, \vec{P}_{2} / \vec{p}_{2}\right)\right] .
\end{array}
$$

To finish the proof, it remains to be shown that the inequality in the last clause above is leftprimitive. This is a rather simple proof by induction on the maximum length of chains in $\Omega$. The base case, when $\Omega$ is the discrete order (hence $P_{1}$ and $P_{2}$ are of the form displayed above), immediately follows from the polarity of $\xi$ and $t$ in $\overrightarrow{p_{1}}$ and $\overrightarrow{p_{2}}$, and by Lemma 49 . The inductive step is routine.

\subsubsection{The Frege axiom in a pre-Heyting algebra setting.}

Let $\mathcal{F}=\varnothing$ and $\mathcal{G}=\{\rightarrow\}$, with $\rightarrow$ binary and of order type $(\partial, 1)$. The logical connectives of the display calculi $\mathbf{D L}$ and $\mathbf{D L} *$ arising from the basic $\mathcal{L}_{\mathrm{DLE}}(\mathcal{F}, \mathcal{G})$-logic can be represented synoptically as follows:

\begin{tabular}{|r|c|c|c|c|c|c|c|c|c|}
\hline Structural symbols & \multicolumn{2}{|c|}{ I } & \multicolumn{2}{|c|}{$;$} & \multicolumn{2}{c|}{$>$} & \multicolumn{2}{c|}{$\succ$} & $\bullet$ \\
\hline Operational symbols & $\top$ & $\perp$ & $\wedge$ & $\vee$ & $(\succ)$ & $(\rightarrow)$ & & $\rightarrow$ & $(\bullet)$ \\
\hline
\end{tabular}

As mentioned in Example 47,

$$
p \rightarrow(q \rightarrow r) \leq(p \rightarrow q) \rightarrow(p \rightarrow r)
$$

\footnotetext{
${ }^{38}$ The formulas $\operatorname{LA}\left(\phi_{1}\right)(\mathbf{i} / u), \operatorname{LA}\left(\phi_{2}\right)(\mathbf{i} / u), \operatorname{RA}\left(\psi_{1}\right)(\mathbf{m} / u)$ and $\operatorname{RA}\left(\psi_{2}\right)(\mathbf{m} / u)$ do not need to be pure, and in general they are not. However, the assumptions and the general theory of ALBA guarantee that they are $\varepsilon^{\partial}$-uniform and free of the variable the 'minimal valuation' of which they are part of. The reader is referred to [18] for an expanded treatment of this point.
} 
is strictly right-primitive $(\Omega, \varepsilon)$-inductive for $r<\Omega p<\Omega q$ and $\varepsilon(p, q, r)=(1,1, \partial)$. Executing ALBA according to this choice of $\Omega$ and $\varepsilon$, we obtain:

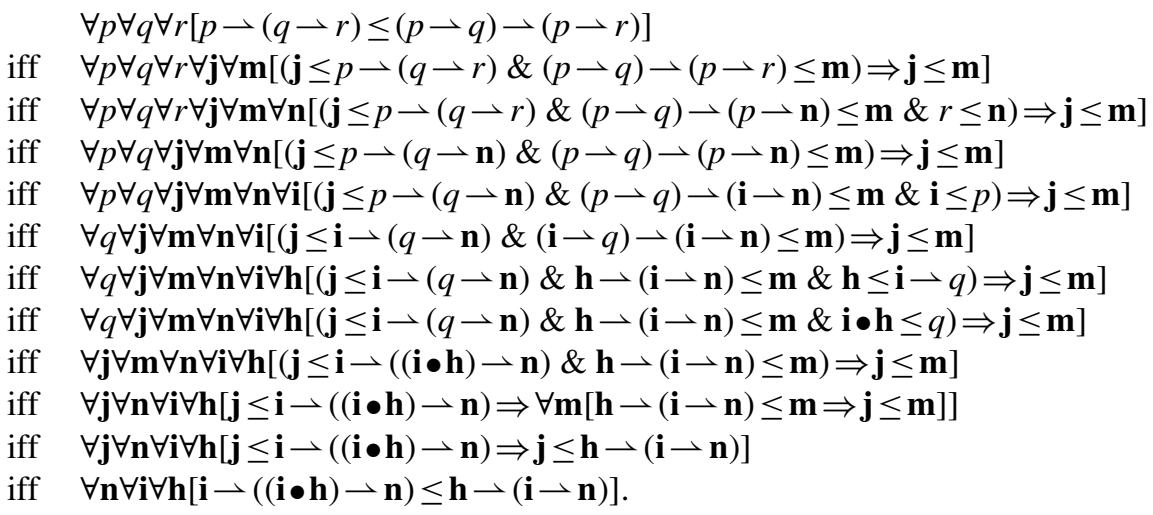

The last inequality above is a pure right-primitive $\mathcal{L}_{D L E}^{*}$-inequality, and by Proposition 37 is equivalent on perfect DLE-algebras to

$$
p \rightarrow((q \bullet p) \rightarrow r) \leq p \rightarrow(q \rightarrow r) .
$$

By applying the usual procedure, we obtain the following rule:

$$
p \rightarrow((q \bullet p) \rightarrow r) \leq p \rightarrow(q \rightarrow r) \quad \rightsquigarrow \frac{x \vdash p \rightarrow((q \bullet p) \rightarrow r)}{x \vdash p \rightarrow(q \rightarrow r)} \quad \rightsquigarrow \quad \frac{X \vdash W \succ((Y \bullet W) \succ Z)}{X \vdash W \succ(Y \succ Z)}
$$

\subsection{Type 4: allowing both sides of inequalities to be non-primitive}

In all syntactic shapes of inequalities treated so far, the tail has been required to be primitive. This requirement is dropped in the syntactic shape treated in the present subsection. Let us start with a motivating example:

\subsubsection{The Church-Rosser inequality}

Let $\mathcal{F}=\{\diamond\}$ and $\mathcal{G}=\{\square\}$. The $\mathcal{L}_{\mathrm{DLE}}(\mathcal{F}, \mathcal{G})$-inequality $\diamond \square p \leq \square \diamond p$ is neither very restricted leftanalytic inductive nor very restricted right-analytic inductive, given that neither side is primitive. However, the following ALBA reduction succeeds in transforming it into a pure left-primitive $\mathcal{L}_{\text {DLE }}^{*}$ inequality:

$\begin{array}{lll} & \forall p[\diamond \square p \leq \square \diamond p] & \\ \text { iff } & \forall p[\diamond \diamond \square p \leq \diamond p] & \text { (Adjunction) } \\ \text { iff } & \forall p \forall \mathbf{i} \forall \mathbf{m}[\mathbf{i} \leq \diamond \diamond \square p \& \diamond p \leq \mathbf{m} \Rightarrow \mathbf{i} \leq \mathbf{m}] & \text { (First approximation) } \\ \text { iff } & \forall p \forall \mathbf{i} \forall \mathbf{j} \forall \mathbf{m}[\mathbf{i} \leq \diamond \diamond \mathbf{j} \& \mathbf{j} \leq \square p \& \diamond p \leq \mathbf{m} \Rightarrow \mathbf{i} \leq \mathbf{m}] & \text { (Approximation rules for } \diamond \text { and } \bullet \text { ) } \\ \text { iff } & \forall p \forall \mathbf{i} \forall \mathbf{j} \forall \mathbf{m}[\mathbf{i} \leq \diamond \mathbf{j} \& \diamond \mathbf{j} \leq p \& \diamond p \leq \mathbf{m} \Rightarrow \mathbf{i} \leq \mathbf{m}] & \text { (Adjunction) } \\ \text { iff } & \forall \mathbf{i} \forall \mathbf{j} \forall \mathbf{m}[\mathbf{i} \leq \diamond \mathbf{j} \& \diamond \diamond \mathbf{j} \leq \mathbf{m} \Rightarrow \mathbf{i} \leq \mathbf{m}] & \text { (Ackermann lemma) } \\ \text { iff } & \forall \mathbf{j}[\diamond \diamond \mathbf{j} \leq \diamond \diamond \mathbf{j}] . & \end{array}$

Notice that this reduction departs in significant ways from the standard ALBA executions as described in Section 3.5, in that we have applied an adjunction rule other than a splitting rule before the first 
approximation step, i.e. as part of the preprocessing, and to a Skeleton node. This rule application is sound, but would be redundant if our goal was restricted to calculating first-order correspondents of input formulas. Notice that this rule application succeeded in transforming the input inequality into the inequality $\diamond \square p \leq \diamond p$, which is very restricted left-analytic inductive (cf. Definition 45), and thus can be treated as indicated in the previous subsection. This example illustrates the ideas on which the treatment of the following class of inequalities is based:

DEFINITION 51 (Restricted analytic inductive inequalities)

For any order type $\varepsilon$ and any irreflexive and transitive relation $\Omega$ on the variables $\vec{p}$, the signed generation tree $* s(* \in\{+,-\})$ of a term $s\left(p_{1}, \ldots p_{n}\right)$ is analytic $(\Omega, \varepsilon)$-inductive if:

(1) $* s$ is $(\Omega, \varepsilon)$-inductive (cf. Definition 16);

(2) every branch of $* s$ is good (cf. Definition 15).

An inequality $s \leq t$ in $\vec{p}$ is restricted left-analytic (resp. right-analytic) $(\Omega, \varepsilon)$-inductive if:

(1) $+s$ (resp. $-t)$ is restricted analytic $(\Omega, \varepsilon)$-inductive (cf. Definition 45$)$ and $-t$ (resp. $+s$ ) is analytic $(\Omega, \varepsilon)$-inductive;

(2) there exists exactly one $\varepsilon^{\partial}$-uniform PIA subtree in $-t$ (resp. in $+s$ ) the root of which is attached to the Skeleton of $-t$ (resp. $+s)$.

An inequality $s \leq t$ is restricted analytic inductive if it is restricted (right-analytic or left-analytic) $(\Omega, \varepsilon)$-inductive for some $\Omega$ and $\varepsilon$.

\section{REMARK 52}

The syntactic shape specified in the definition above can be intuitively understood with the help of the following picture, which illustrates the 'left-analytic' case:
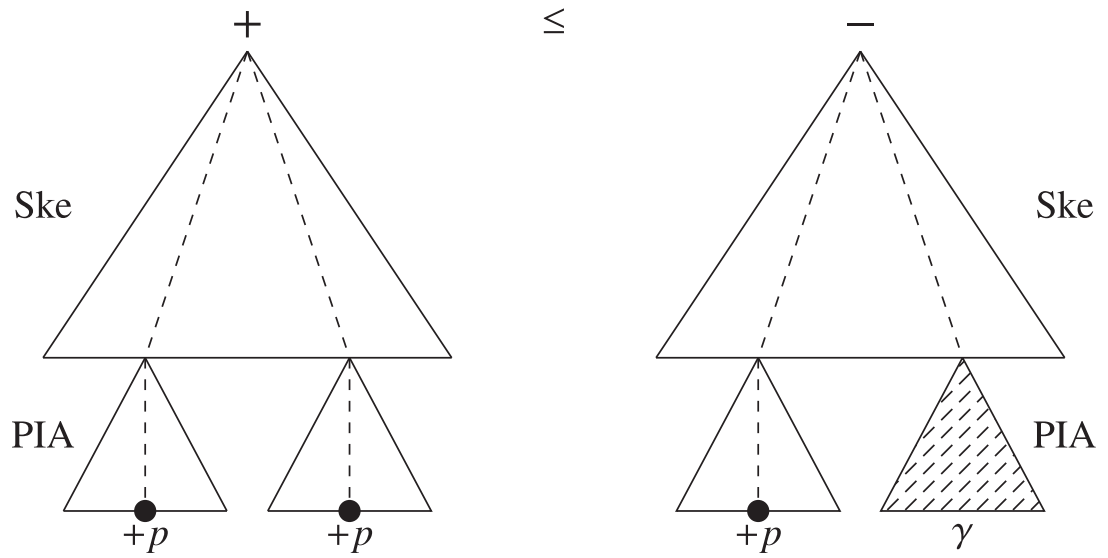

As the picture shows, similarly to the very restricted analytic inductive inequalities, this syntactic shape forbids the root of any $\varepsilon^{\partial}$-uniform subtree to be attached directly to the skeleton of the head of the inequality. However, in contrast to the very restricted analytic inductive inequalities, critical branches can appear now in the tail of the inequality. Finally, there exists a unique $\varepsilon^{\partial}$-uniform subtree whose root is attached to the skeleton of the tail of the inequality. In the lemma below, we will denote the tail of a restricted left-analytic (resp. right-analytic) inductive inequality by $\xi(\gamma / ! x, \vec{\psi} / \bar{z})$, where $\xi(! x, \bar{z})$ is a negative (resp. positive) skeleton term, and $\gamma$ denotes the unique $\varepsilon^{\partial}$-uniform PIA subtree attached to the skeleton, and for each $\psi \in \vec{\psi}$ with $* \psi \prec-\xi$ is a PIA subtree that contains a critical branch. 


\subsubsection{BNF presentation of analytic $(\Omega, \varepsilon)$-inductive terms}

In what follows, we adopt the following conventions: when writing e.g. $g(\vec{x}, \vec{y}, \vec{z}, \vec{w})$, we understand that the arrays of variables are of different lengths, which can be possibly 0 , and moreover $g$ is monotone in $\vec{x}$ and $\vec{z}$ and is antitone in $\vec{y}$ and $\vec{w}$. Let us first introduce the Backus-Naur Form (BNF) presentation of the $\varepsilon^{\partial}$-uniform PIA terms $\gamma$ and $\chi$, which are substituted for positive and negative placeholder variables in the skeleton of analytic inductive terms, respectively. This implies that we will only be interested in the signed generation trees $+\gamma$ and $-\chi$. Moreover, we will use the letter $p$ (or $\vec{p}$ ) to indicate those variables which are assigned to 1 by $\varepsilon$, and the letter $q$ (or $\vec{q}$ ) for those which are assigned to $\partial$.

$$
\begin{aligned}
& \gamma:=q|\perp| \top|\gamma \vee \gamma| \gamma \wedge \gamma \mid g(\vec{\gamma} / \vec{x}, \vec{\chi} / \vec{y}), \\
& \chi:=p|\top| \perp|\chi \wedge \chi| \chi \vee \chi \mid f(\vec{\chi} / \vec{x}, \vec{\gamma} / \vec{y}) .
\end{aligned}
$$

Next, let us introduce the BNF presentation of the non $\varepsilon^{\partial}$-uniform PIA terms $\phi$ and $\psi$, which are substituted for positive and negative placeholder variables in the skeleton of analytic inductive terms, respectively. This implies that we will only be interested in the signed generation trees $+\phi$ and $-\psi$. Let PosPIA and NegPIA, respectively, denote the sets of the $\phi$ - and $\psi$-terms. In addition, we will need - and define by simultaneous induction - the function

$$
\text { CVar:PosPIA } \cup \text { NegPIA } \rightarrow \wp(\text { Var })
$$

which maps each $\phi$ and $\psi$ to the set of variables of which there are critical occurrences in $\phi$ and $\psi$.

$$
\begin{gathered}
\phi:=p|\top \wedge \phi| \perp \wedge \phi|\phi \wedge \phi| \gamma \vee \phi|g(\vec{\gamma} / \vec{x}, \vec{\chi} / \vec{y}, \phi / z)| g(\vec{\gamma} / \vec{x}, \vec{\chi} / \vec{y}, \psi / w) \\
\psi:=q|\perp \vee \psi| \top \wedge \psi|\psi \vee \psi| \chi \wedge \psi|f(\vec{\chi} / \vec{x}, \vec{\gamma} / \vec{y}, \psi / z)| f(\vec{\chi} / \vec{x}, \vec{\gamma} / \vec{y}, \phi / w) .
\end{gathered}
$$

In the two presentations above, the construction of the terms which have $g$ or $f$ as their main connectives is subject to the condition that all the variables in $C \operatorname{Var}(\phi)(\operatorname{resp} . \mathrm{CVar}(\psi))$-where $\phi$ and $\psi$ denote the immediate subformulas as indicated above-are common upper bounds of the variables occurring in $\vec{\gamma}$ and $\vec{\chi}$ w.r.t. $\Omega$.

$$
\begin{aligned}
& \operatorname{CVar}(p)=\{p\} \\
& \mathrm{C} \operatorname{Var}\left(\phi_{1} \wedge \phi_{2}\right)=\mathrm{C} \operatorname{Var}\left(\phi_{1}\right) \cup \mathrm{C} \operatorname{Var}\left(\phi_{2}\right) \\
& \operatorname{CVar}(g(\vec{\gamma} / \vec{x}, \vec{\chi} / \vec{y}, \phi / z))=\operatorname{CVar}(\phi) \\
& \mathrm{CVar}(g(\vec{\gamma} / \vec{x}, \vec{\chi} / \vec{y}, \psi / w))=\mathrm{C} \operatorname{Var}(\psi) \\
& \begin{aligned}
\mathrm{C} \operatorname{Var}(q) & =\{q\} \\
\mathrm{C} \operatorname{Var}\left(\psi_{1} \vee \psi_{2}\right) & =\mathrm{C} \operatorname{Var}\left(\psi_{1}\right) \cup \operatorname{CVar}\left(\psi_{2}\right) \\
\mathrm{C} \operatorname{Var}(f(\vec{\chi} / \vec{x}, \vec{\gamma} / \vec{y}, \psi / z)) & =\mathrm{CVar}(\psi) \\
\mathrm{C} \operatorname{Var}(f(\vec{\chi} / \vec{x}, \vec{\gamma} / \vec{y}, \phi / w)) & =\mathrm{C} \operatorname{Var}(\phi)
\end{aligned}
\end{aligned}
$$

Finally, let us introduce the BNF presentation of the analytic inductive terms $s$ and $t$, which are to occur on the left-hand side and right-hand side of inequalities, respectively. This implies that we will only be interested in the sign generation trees $+s$ and $-t$.

$$
\begin{aligned}
& s:=\gamma|\phi| s \vee s|s \wedge s| f(\vec{s} / \vec{x}, \vec{t} / \vec{y}), \\
& t:=\chi|\psi| t \wedge t|t \vee t| g(\vec{t} / \vec{x}, \vec{s} / \vec{y}) .
\end{aligned}
$$

LEMMA 53

For any $\mathcal{L}_{\text {DLE}}$-inequality $s \leq t$ :

(1) If $s \leq t=\xi(\gamma / ! x, \vec{\psi} / \bar{z})$ is restricted left-analytic $(\Omega, \varepsilon)$-inductive such that $\xi$ is definite and $-x \prec-\xi$ (resp. $+x \prec-\xi$ ), the adjunction rule $\mathrm{LA}(\xi)$ is applicable and yields the equivalent inequality $\operatorname{LA}(\xi)(s / u, \vec{\psi}) \leq \gamma$ (resp. $\gamma \leq \operatorname{LA}(\xi)(s / u, \vec{\psi}))$, which is very restricted left-analytic (resp. right-analytic) $(\Omega, \varepsilon)$-inductive. 
(2) If $\xi(\gamma / ! x, \vec{\psi} / \bar{z})=s \leq t$ is restricted right-analytic $(\Omega, \varepsilon)$-inductive such that $\xi$ is definite and $+x \prec+\xi$ (resp. $-x \prec+\xi$ ), the adjunction rule $\mathrm{RA}(\xi)$ is applicable and yields the equivalent inequality $\gamma \leq \operatorname{RA}(\xi)(t / u, \vec{\psi})(\operatorname{resp} . \operatorname{RA}(\xi)(t / u, \vec{\psi}) \leq \gamma)$, which is very restricted right-analytic (resp. left-analytic) $(\Omega, \varepsilon)$-inductive.

Proof. We only show the first item in the case $-x \prec-\xi$, the remaining cases being similar. The assumptions imply (cf. Lemma 49) that the rule $L A(\xi)$ is applicable to $s \leq t$ so as to obtain the inequality $\operatorname{LA}(\xi)(s / u, \vec{\psi}) \leq \gamma$, and that $\operatorname{LA}(\xi)(s / u, \bar{z})$ is a definite negative PIA formula. Since the polarities of $\bar{z}$ do not change under the application of adjunction rules and the polarity of $u$ is positive, in $\operatorname{LA}(\xi)(s / u, \vec{\psi})$ the subtree of each $\psi \in \vec{\psi}$ remains a PIA subtree with at least one critical branch, and the branches running through $s$ remain good. Hence, $+\operatorname{LA}(\xi)(s / u, \vec{\psi})$ is $(\Omega, \varepsilon)$-inductive, all of its branches are good, and all of its maximal $\varepsilon^{\partial}$-uniform PIA subtrees occur as immediate subtrees of SRR nodes of some $\varepsilon$-critical branches. That is, $\operatorname{LA}(\xi)(s / u, \vec{\psi})$ is a restricted analytic inductive term. Furthermore, $\gamma$ is negative PIA, and $-\gamma$ is $\varepsilon^{\partial}$-uniform by assumption. From the above observations it follows that $\operatorname{LA}(\xi)(s / u, \vec{\psi}) \leq \gamma$ is a very restricted left-analytic $(\Omega, \varepsilon)$-inductive inequality.

\section{COROLlary 54}

Every restricted left-analytic (resp. right-analytic) inductive $\mathcal{L}_{\text {DLE-inequality can be equivalently }}$ transformed, via an ALBA-reduction, into a set of pure left-primitive (resp. right-primitive) $\mathcal{L}_{\mathrm{DLE}^{*}}$ inequalities.

ProOF. We only consider the case of the inequality $s \leq t=\xi(\gamma / ! x, \vec{\psi} / \bar{z})$ being restricted left-analytic $(\Omega, \varepsilon)$-inductive, and $+x \prec-\xi$, the remaining cases being similar. Modulo exhaustive application of distribution and splitting rules of the standard ALBA preprocessing,${ }^{39}$ we can assume w.l.o.g. that the negative Skeleton formula $\xi$ is also definite. By Lemma 53, the adjunction rule $\mathrm{LA}(\xi)$ is applicable and yields the equivalent inequality $\gamma \leq \operatorname{LA}(\xi)(s / u, \vec{\psi})$, which is very restricted right-analytic $(\Omega, \varepsilon)$ inductive. Hence, the statement follows by Theorem 50 .

\subsubsection{The Frege inequality, again}

Early on (cf. Section 6.2.1), we have discussed the Frege inequality as an example of very restricted right-analytic $(\Omega, \varepsilon)$-inductive inequality for $r<\Omega p<\Omega q$ and $\varepsilon(p, q, r)=(1,1, \partial)$. Here below, we provide an alternative solving strategy based on the fact that the Frege inequality is also a restricted left-analytic $(\Omega, \varepsilon)$-inductive inequality for $\varepsilon(p, q, r)=(1,1,1)$.

$$
\begin{array}{lll} 
& \forall p \forall q \forall r[p \rightarrow(q \rightarrow r) \leq(p \rightarrow q) \rightarrow(p \rightarrow r)] & \\
\text { iff } & \forall p \forall q \forall r[(p \rightarrow q) \bullet(p \rightarrow(q \rightarrow r)) \leq p \rightarrow r] & \text { (Residuation) } \\
\text { iff } & \forall p \forall q \forall r[p \bullet((p \rightarrow q) \bullet(p \rightarrow(q \rightarrow r))) \leq r] & \text { (Residuation) } \\
\text { iff } & \forall p \forall q \forall r \forall \mathbf{i} \forall \mathbf{m}[\mathbf{i} \leq p \bullet((p \rightarrow q) \bullet(p \rightarrow(q \rightarrow r))) \& r \leq \mathbf{m} \Rightarrow \mathbf{i} \leq \mathbf{m}] & \text { (First approximation) } \\
\text { iff } & \forall p \forall q \forall r \forall \mathbf{i} \forall \mathbf{j} \forall \mathbf{k} \forall \mathbf{h} \forall \mathbf{m}[\mathbf{i} \leq \mathbf{j} \bullet(\mathbf{k} \bullet \mathbf{h}) \& & \\
& \mathbf{j} \leq p \& \mathbf{k} \leq p \rightarrow q \& \mathbf{h} \leq p \rightarrow(q \rightarrow r) \& r \leq \mathbf{m} \Rightarrow \mathbf{i} \leq \mathbf{m}] & \text { (Approximation) } \\
\text { iff } & \forall p \forall q \forall r \forall \mathbf{i} \forall \mathbf{j} \forall \mathbf{k} \forall \mathbf{h} \forall \mathbf{m}[\mathbf{i} \leq \mathbf{j} \bullet(\mathbf{k} \bullet \mathbf{h}) \& & \\
& \mathbf{j} \leq p \& p \bullet \mathbf{k} \leq q \& q \bullet(p \bullet \mathbf{h}) \leq r \& r \leq \mathbf{m} \Rightarrow \mathbf{i} \leq \mathbf{m}] & \text { (Residuation) }
\end{array}
$$

\footnotetext{
${ }^{39}$ The applications of splitting rules at this stage give rise to a set of inequalities, each of which can be treated separately. In the remainder of the proof, we focus on one of them.
} 


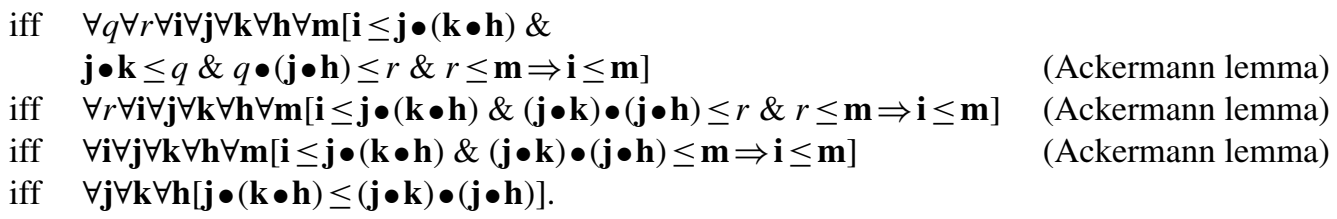

The last inequality above is a pure left-primitive $\mathcal{L}_{\mathrm{DLE}}^{*}$-inequality, and by Proposition 37 is equivalent on perfect DLE-algebras to

$$
p \bullet(q \bullet r) \leq(p \bullet q) \bullet(p \bullet r) .
$$

By applying the usual procedure, we obtain the following rule:

$$
p \bullet(q \bullet r) \leq(p \bullet q) \bullet(p \bullet r) \rightsquigarrow \frac{(p \bullet q) \bullet(p \bullet r) \vdash y}{p \bullet(q \bullet r) \vdash y} \rightsquigarrow \frac{(X \bullet Y) \bullet(X \bullet Z) \vdash W}{X \bullet(Y \bullet Z) \vdash W}
$$

\subsubsection{The non-primitive Fischer Servi inequality}

For the $\mathcal{L}_{\text {DLE}}$-setting specified as in Example 34, the Fischer Servi inequality $\diamond(p \rightarrow q) \leq \square p \rightarrow \diamond q$ is restricted right-analytic $(\Omega, \varepsilon)$-inductive w.r.t. the discrete order $\Omega$ and $\varepsilon(p, q)=(1, \partial)$. Let us apply the procedure indicated in the proof of Corollary 54 to it:

$$
\begin{aligned}
& \forall p \forall q[\diamond(p \rightarrow q) \leq \square p \rightarrow \diamond q] \\
& \text { iff } \forall p \forall q[p \rightarrow q \leq \mathbf{\square}(\square p \rightarrow \diamond q)] \\
& \text { iff } \quad \forall p \forall q \forall \mathbf{i} \forall \mathbf{m}[(\mathbf{i} \leq p \rightarrow q \& \mathbf{m}(\square p \rightarrow \diamond q) \leq \mathbf{m}) \Rightarrow \mathbf{i} \leq \mathbf{m}] \\
& \text { iff } \forall p \forall q \forall \mathbf{i} \forall m \forall \mathbf{j} \forall \mathbf{n}[(\mathbf{i} \leq p \rightarrow q \& \mathbf{\square}(\mathbf{j} \rightarrow \mathbf{n}) \leq \mathbf{m} \& \mathbf{j} \leq \square p \& \diamond q \leq \mathbf{n}) \Rightarrow \mathbf{i} \leq \mathbf{m}] \quad \text { (Approximation) } \\
& \text { iff } \quad \forall p \forall q \forall \mathbf{i} \forall m \forall \mathbf{j} \forall \mathbf{n}[(\mathbf{i} \leq p \rightarrow q \& \mathbf{\square}(\mathbf{j} \rightarrow \mathbf{n}) \leq \mathbf{m} \& \bullet \mathbf{j} \leq p \& q \leq \mathbf{m}) \Rightarrow \mathbf{i} \leq \mathbf{m}] \quad \text { (Adjunction) } \\
& \text { iff } \forall \mathbf{i} \forall m \forall \mathbf{j} \forall \mathbf{n}[(\mathbf{i} \leq \bullet \mathbf{j} \rightarrow \mathbf{m} \& \mathbf{n}(\mathbf{j} \rightarrow \mathbf{n}) \leq \mathbf{m}) \Rightarrow \mathbf{i} \leq \mathbf{m}] \quad \text { (Ackermann) } \\
& \text { iff } \forall \mathbf{j} \forall \mathbf{n}[\bullet \mathbf{j} \rightarrow \mathbf{\square n} \leq \mathbf{a}(\mathbf{j} \rightarrow \mathbf{n})] \text {. }
\end{aligned}
$$

The last inequality above is a pure right-primitive $\mathcal{L}_{\mathrm{DLE}}^{*}$-inequality, and by Proposition 37 is equivalent on perfect DLE-algebras to

$$
\bullet p \rightarrow \mathbf{q} q \mathbf{\mathbf { a }}(p \rightarrow q)
$$

By applying the usual procedure, we obtain the following rule:

$$
\bullet p \rightarrow q \leq \mathbf{\bullet}(p \rightarrow q) \quad \rightsquigarrow \quad \frac{x \vdash \bullet \rightarrow-q}{x \vdash \bullet(p \rightarrow q)} \rightsquigarrow \frac{X \vdash \bullet Y>\bullet Z}{X \vdash \bullet(Y>Z)}
$$

\subsubsection{The 'transitivity' axiom}

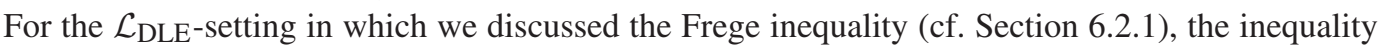
$(p \rightarrow q) \bullet(q \rightarrow r) \leq p \rightarrow r$ is restricted right-analytic $(\Omega, \varepsilon)$-inductive w.r.t. the order $p<\Omega q$ and 
$\varepsilon(p, q, r)=(1, \partial, \partial)$. Let us apply the procedure indicated in the proof of Corollary 54 to it:

$$
\begin{array}{lll} 
& \forall p \forall q \forall r[(p \rightarrow q) \bullet(q \rightarrow r) \leq p \rightarrow r] & \\
\text { iff } \quad \forall p \forall q \forall r[q \rightarrow r \leq(p \rightarrow q) \rightarrow(p \rightarrow r)] & \text { (Adjunction) } \\
\text { iff } \quad \forall p q r \forall \mathbf{i} \forall \mathbf{m}[(\mathbf{i} \leq q \rightarrow r \&(p \rightarrow q) \rightarrow(p \rightarrow r) \leq \mathbf{m}) \Rightarrow \mathbf{i} \leq \mathbf{m}] & \text { (First approximation } \\
\text { iff } \quad \forall p q r \forall \mathbf{i m h j n}[(\mathbf{i} \leq q \rightarrow r \& \mathbf{j} \rightarrow(\mathbf{h} \rightarrow \mathbf{n}) \leq \mathbf{m} \& \mathbf{h} \leq p \& r \leq \mathbf{n} \& \mathbf{j} \leq p \rightarrow q) \Rightarrow \mathbf{i} \leq \mathbf{m}] & \text { (Approximation) } \\
\text { iff } \quad \forall q \forall \mathbf{i} \forall \mathbf{m} \forall \mathbf{h} \forall \mathbf{j} \forall \mathbf{n}[(\mathbf{i} \leq q \rightarrow \mathbf{n} \& \mathbf{j} \rightarrow(\mathbf{h} \rightarrow \mathbf{n}) \leq \mathbf{m} \& \mathbf{j} \leq \mathbf{h} \rightarrow q) \Rightarrow \mathbf{i} \leq \mathbf{m}] & \text { (Ackermann) } \\
\text { iff } \quad \forall q \forall \mathbf{i} \forall \mathbf{m} \forall \mathbf{h} \forall \mathbf{j} \forall \mathbf{n}[(\mathbf{i} \leq q \rightarrow \mathbf{n} \& \mathbf{j} \rightarrow(\mathbf{h} \rightarrow \mathbf{n}) \leq \mathbf{m} \& \mathbf{h} \bullet \mathbf{j} \leq q) \Rightarrow \mathbf{i} \leq \mathbf{m}] & \text { (Adjunction) } \\
\text { iff } \quad \forall \mathbf{i} \forall \mathbf{m} \forall \mathbf{h} \forall \mathbf{j} \forall \mathbf{n}[(\mathbf{i} \leq(\mathbf{h} \bullet \mathbf{j}) \rightarrow \mathbf{n} \& \mathbf{j} \rightarrow(\mathbf{h} \rightarrow \mathbf{n}) \leq \mathbf{m}) \Rightarrow \mathbf{i} \leq \mathbf{m}] & \text { (Ackermann) } \\
\text { iff } \quad \forall \mathbf{h} \forall \mathbf{j} \forall \mathbf{n}[(\mathbf{h} \bullet \mathbf{j}) \rightarrow \mathbf{n} \leq \mathbf{j} \rightarrow(\mathbf{h} \rightarrow \mathbf{n})] . &
\end{array}
$$

The last inequality above is a pure right-primitive $\mathcal{L}_{\mathrm{DLE}}^{*}$-inequality, and by Proposition 37 is equivalent on perfect DLE-algebras to

$$
(p \bullet q) \rightarrow r \leq q \rightarrow(p \rightarrow r) .
$$

By applying the usual procedure, we obtain the following rule:

$$
(p \bullet q) \rightarrow r \leq q \rightarrow(p \rightarrow r) \quad \rightsquigarrow \frac{x \vdash(p \bullet q) \rightarrow r}{x \vdash q \rightarrow(p \rightarrow r)} \quad \rightsquigarrow \frac{X \vdash(Y \bullet Z) \succ W}{X \vdash Z \succ(Y \succ W)}
$$

\section{Analytic inductive inequalities and analytic rules}

In the present section, we address the most general syntactic shape considered in the article: in the following subsection, we define the class of analytic inductive inequalities, and show that each of them can be equivalently transformed into (a set of) analytic structural rules (which are in fact quasispecial). In Section 7.2, we also show that any analytic rule is semantically equivalent to some analytic inductive inequality. Thus, the DLE-logics axiomatized by means of analytic inductive inequalities are exactly the properly displayable ones.

\subsection{From analytic inductive inequalities to quasi-special rules}

Let us start with a motivating example:

\subsubsection{The pre-linearity axiom}

Let $\mathcal{F}=\varnothing, \mathcal{G}=\{\rightarrow\}$ where $\rightarrow$ is binary and of order type $(\partial, 1)$.

\begin{tabular}{|c|c|c|c|c|c|}
\hline \multicolumn{2}{|c|}{$\mathrm{I}$} & \multicolumn{2}{|c|}{$;$} & \multicolumn{2}{c|}{$\succ$} \\
\hline $\mathrm{T}$ & $\perp$ & $\wedge$ & $\vee$ & & $\rightarrow$ \\
\hline
\end{tabular}

The following inequality

$$
\top \leq(p \rightarrow q) \vee(q \rightarrow p)
$$

is not restricted analytic inductive for any order type: indeed, all the non-leaf nodes of the right hand are Skeleton, and the PIA subterms are reduced to the variables. The inequality above is not restricted right-analytic for any order type $\varepsilon$, since the right hand side contains $\varepsilon^{\partial}$-uniform PIA-subterms attached to the skeleton. It is not restricted left-analytic for any order type $\varepsilon$, since the right-hand side contains more than one $\varepsilon^{\partial}$-uniform PIA-subterm. 
We have not found an ALBA-reduction suitable to extend the strategy of the previous section so as to equivalently transform the inequality above into one or more primitive inequalities. However, the following ALBA reduction, exclusively based on applications of a modified (inverted) Ackermann rule (the soundness of which is proved in Lemma 57 below) and adjunction rules, transforms the inequality above into a quasi-inequality which gives rise to an analytic (in fact quasi-special, cf. Definition 8) structural rule.

$$
\begin{aligned}
& \forall p \forall q[\top \leq(p-q) \vee(q \rightarrow p)] \\
& \forall p \forall q \forall \vec{r}\left[\left(r_{1} \leq p \& q \leq r_{2} \& r_{3} \leq q \& p \leq r_{4}\right) \Rightarrow \top \leq\left(r_{1} \rightarrow r_{2}\right) \vee\left(r_{3} \rightarrow r_{4}\right)\right] \\
& \forall q \forall \vec{r}\left[\left(r_{1} \leq r_{4} \& q \leq r_{2} \& r_{3} \leq q\right) \Rightarrow \top \leq\left(r_{1} \rightarrow r_{2}\right) \vee\left(r_{3} \rightarrow r_{4}\right)\right] \\
& \forall \vec{r}\left[\left(r_{1} \leq r_{4} \& r_{3} \leq r_{2}\right) \Rightarrow \top \leq\left(r_{1} \rightarrow r_{2}\right) \vee\left(r_{3} \rightarrow r_{4}\right)\right] .
\end{aligned}
$$

The last quasi-inequality above expresses the validity of the following quasi-special structural rule on perfect DLEs:

$$
\frac{X \vdash W \quad Z \vdash Y}{\mathrm{I} \vdash(X \succ Y) ;(Z \succ W)}
$$

We will see that the solving strategy applied to the example above can be applied to the following class of inequalities:

DEFINITION 55 (Analytic inductive inequalities)

For every order type $\varepsilon$ and every irreflexive and transitive relation $\Omega$ on the variables $p_{1}, \ldots p_{n}$, an inequality $s \leq t$ is analytic $(\Omega, \varepsilon)$-inductive if $+s$ and $-t$ are both $(\Omega, \varepsilon)$-analytic inductive (cf. Definition 51). An inequality $s \leq t$ is analytic inductive if is $(\Omega, \varepsilon)$-analytic inductive for some $\Omega$ and $\varepsilon$.

\section{REMARK 56}

The syntactic shape specified in the definition above can be intuitively understood with the help of the following picture:
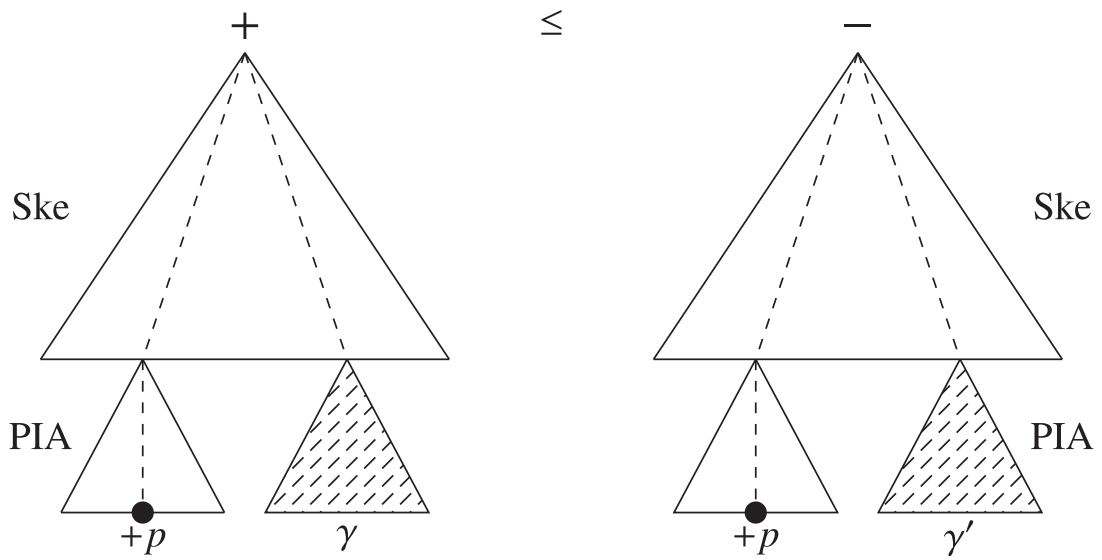

As the picture shows, the difference between analytic inductive inequalities and restricted analytic inductive inequalities is that, in the latter, there can be exactly one $\varepsilon^{\partial}$-uniform subterm attached to the skeleton of the inequality, while in the former this requirement is dropped.

Below, we discuss a slightly modified version of the Ackermann rule, which will be used in the proof of Proposition 59. 


\section{LEMMA 57}

Let $s(\overrightarrow{! q})$ and $t(\overrightarrow{! r})$ be $\mathcal{L}_{\mathrm{DLE}}$-terms such that the propositional variables in the arrays $\overrightarrow{! q}$ and $\overrightarrow{! r}$ are disjoint. Let $\varepsilon$ be the unique order type on the concatenation $\overrightarrow{!} q \oplus \overrightarrow{!} r$ with respect to which $s \leq t$ is $\varepsilon$-uniform. Let $\vec{\alpha}$ and $\vec{\beta}$ be arrays of DLE-terms of the same length as $\vec{q}$ and $\vec{r}$, respectively, and such that no variable in $\vec{q} \oplus \vec{r}$ occurs in any $\alpha$ or $\beta$. Then the following are equivalent for any perfect DLE $\mathbb{A}$ :

(1) $\mathbb{A} \models s(\vec{\alpha} / \vec{q}) \leq t(\vec{\beta} / \vec{r})$;

(2) $\mathbb{A} \models \forall \vec{q} \forall \vec{r}\left[\&_{q \in \vec{q}, r \in \vec{r}}\left(q \leq^{\varepsilon(q)} \alpha \& \beta \leq^{\varepsilon(r)} r\right) \Rightarrow s(\overrightarrow{! q}) \leq t(\overrightarrow{!})\right]$,

where $\leq^{1}:=\leq$ and $\leq^{\partial}:=\geq$.

Proof. Let us assume (1). To show (2), fix an interpretation $v$ of the variables in $\mathbb{A}$ such that $(\mathbb{A}, v) \models q \leq^{\varepsilon(q)} \alpha$ and $(\mathbb{A}, v) \models \beta \leq^{\varepsilon(r)} r$ for each $q$ in $\vec{q}$ and $r$ in $\vec{r}$. Hence, assumption (1) and the $\varepsilon$-uniformity of $s \leq t$ imply that $\llbracket s(\vec{q}) \rrbracket_{v} \leq \llbracket s(\vec{\alpha} / \vec{q}) \rrbracket_{v} \leq \llbracket t(\vec{\beta} / \vec{r}) \rrbracket_{v} \leq \llbracket t(\vec{r}) \rrbracket_{v}$, which proves that $(\mathbb{A}, v) \models$ $s(\vec{q}) \leq t(\vec{r})$, as required. Conversely, fix a valuation $v$, and notice that the truth of the required condition $(\mathbb{A}, v) \models s(\vec{\alpha} / \vec{q}) \leq t(\vec{\beta} / \vec{r})$ does not depend on where $v$ maps the variables in $\vec{q} \oplus \vec{r}$, since none of these variables occurs in $s(\vec{\alpha} / \vec{q}) \leq t(\vec{\beta} / \vec{r})$. Hence, it is enough to show that $\left(\mathbb{A}, v^{\prime}\right) \models s(\vec{\alpha} / \vec{q}) \leq t(\vec{\beta} / \vec{r})$ for some $\vec{q} \oplus \vec{r}$-variant $v^{\prime}$ of $v$. Let $v^{\prime}$ be the $\vec{q} \oplus \vec{r}$-variant of $v$ such that $\llbracket q_{i} \rrbracket_{v^{\prime}}:=\llbracket \alpha_{i} \rrbracket_{v}=\llbracket \alpha_{i} \rrbracket_{v^{\prime}}$ and $\llbracket r_{j} \rrbracket_{v^{\prime}}:=$ $\llbracket \beta_{j} \rrbracket_{v}=\llbracket \beta_{j} \rrbracket_{v^{\prime}}$. Then clearly, $\left(\mathbb{A}, v^{\prime}\right) \models q \leq^{\varepsilon(q)} \alpha$ and $\left(\mathbb{A}, v^{\prime}\right) \models \beta \leq^{\varepsilon(r)} r$. By assumption 2 , this implies that $\left(\mathbb{A}, v^{\prime}\right) \models s(\vec{q}) \leq t(\vec{r})$, which is equivalent by construction to the required $\left(\mathbb{A}, v^{\prime}\right) \models s(\vec{\alpha} / \vec{q}) \leq t(\vec{\beta} / \vec{r})$.

\section{REMARK 58}

Notice that, in the quasi-inequality in item (2) of the statement of the lemma above, each variable $q$ in $\vec{q}$ and $r$ in $\vec{r}$ occurs twice, i.e. once in exactly one inequality in the antecedent and once in the conclusion of the quasi-inequality. These two occurrences have the same polarity in the two inequalities. For example, if $q$ is in $\vec{q}_{1}$ and $\varepsilon(q)=\partial$, then $q$ occurs negatively in the conclusion of the quasi-inequality, and also negatively in the inequality $q \leq^{\varepsilon(q)} \phi$, which can be rewritten as $\phi \leq q$. The remaining cases are analogous and left to the reader.

\section{Proposition 59}

Every analytic $(\Omega, \varepsilon)$-inductive inequality can be equivalently transformed, via an ALBA-reduction, into a set of quasi-special structural rules.

Proof. By assumption, $s \leq t$ is of the form

$$
\xi_{1}\left(\vec{\phi}_{1} / \vec{x}_{1}, \vec{\psi}_{1} / \vec{y}_{1}, \vec{\gamma}_{1} / \vec{z}_{1}, \vec{\chi}_{1} / \vec{w}_{1}\right) \leq \xi_{2}\left(\vec{\psi}_{2} / \vec{x}_{2}, \vec{\phi}_{2} / \vec{y}_{2}, \vec{\chi}_{2} / \vec{z}_{2}, \vec{\gamma}_{2} / \vec{w}_{2}\right)
$$

where $\xi_{1}\left(\vec{l}_{1}, \overrightarrow{!} y_{1}, \overrightarrow{!} z_{1}, \vec{w}_{1}\right)$ and $\xi_{2}\left(\vec{l}_{2}, \overrightarrow{!} y_{2}, \vec{l}_{2}, \vec{w}_{2}\right)$, respectively, are a positive and a negative Skeleton formula (cf. paragraph after Definition 16) which are scattered, monotone in $\vec{x}$ and $\vec{z}$ and antitone in $\vec{y}$ and $\vec{w}$. Moreover, the formulas in $\vec{\phi}$ and $\vec{\gamma}$ are positive PIA, and the formulas in $\vec{\psi}$ and $\vec{\chi}$ are negative PIA. Finally, every $\phi$ and $\psi$ contains at least one $\varepsilon$-critical variable, whereas all $+\gamma$ and $-\chi$ are $\varepsilon^{\partial}$-uniform. Modulo exhaustive application of distribution and splitting rules of the standard ALBA preprocessing ${ }^{40}$ we can assume w.l.o.g. that the scattered Skeleton formulas $\xi_{1}$ and $\xi_{2}$ are also definite. Modulo exhaustive application of the additional rules which identify $+f^{\prime}\left(\phi_{1}, \ldots, \perp^{\varepsilon_{f^{\prime}}}(i), \ldots, \phi_{n_{f^{\prime}}}\right)$ with

\footnotetext{
${ }^{40}$ The applications of splitting rules at this stage give rise to a set of inequalities, each of which can be treated separately. In the remainder of the proof, we focus on one of them.
} 
$+\perp$ for every $f^{\prime} \in \mathcal{F} \cup\{\wedge\}$ and $-g^{\prime}\left(\phi_{1}, \ldots, \top^{\varepsilon_{g^{\prime}}}(i), \ldots, \phi_{n_{g^{\prime}}}\right)$ with $-\top$ for every $g^{\prime} \in \mathcal{G} \cup\{\vee\}$, which would reduce $s \leq t$ to a tautology, we can assume w.l.o.g. that there are no occurrences of $+\perp$ and $-\top$ in $+\xi_{1}$ and $-\xi_{2}$. Hence, (cf. Remark 29) we can assume w.l.o.g. that $\xi_{1}$ (resp. $\xi_{2}$ ) is scattered, definite and left-primitive (resp. right-primitive). The following equivalence is justified by Lemma 57 (in what follows, we write e.g. $\vec{q}_{1,6} \leq \vec{\phi}$ to represent concisely both $\vec{q}_{1} \leq \vec{\phi}_{1}$ and $\vec{q}_{6} \leq \vec{\phi}_{2}$ ):

$$
\text { iff } \quad \forall \vec{p} \forall \vec{q}\left[\left(\vec{q}_{1,6} \leq \vec{\phi} \& \vec{\psi} \leq \vec{q}_{2,5} \& \vec{q}_{3,8} \leq \vec{\gamma} \& \vec{\chi} \leq \vec{q}_{4,7}\right) \Rightarrow \xi_{1}\left(\vec{q}_{1}, \vec{q}_{2}, \vec{q}_{3}, \vec{q}_{4}\right) \leq \xi_{2}\left(\vec{q}_{5}, \vec{q}_{6}, \vec{q}_{7}, \vec{q}_{8}\right)\right] .
$$

By assumption, in each inequality $q \leq \phi$ (resp. $\psi \leq q$ ) in $\vec{q}_{1,6} \leq \vec{\phi}$ (resp. $\vec{\psi} \leq \vec{q}_{2,5}$ ) there is at least one $\varepsilon$-critical occurrence of some variable in $\vec{p}$. Modulo exhaustive application of distribution rules (a')-(c') of Remark 18 and splitting rules, we can assume w.l.o.g. that each $\phi$ in $\vec{\phi}$ (resp. $\psi$ in $\vec{\psi}$ ) is a definite positive (resp. negative) PIA-formula, which has exactly one $\varepsilon$-critical variable occurrence. That is, if $\overrightarrow{p_{1}}$ and $\overrightarrow{p_{2}}$, respectively, denote the subarrays of $\vec{p}$ such that $\varepsilon\left(p_{1}\right)=1$ for each $p_{1}$ in $\overrightarrow{p_{1}}$ and $\varepsilon\left(p_{2}\right)=\partial$ for each $p_{2}$ in $\overrightarrow{p_{2}}$, then each $\phi$ in $\vec{\phi}$ is either of the form $\phi_{+}\left(p_{1} / ! x, \overrightarrow{p^{\prime}} / \bar{z}\right)$ with $+x \prec+\phi_{+}$, or of the form $\phi_{-}\left(p_{2} / ! x, \overrightarrow{p^{\prime}} / \bar{z}\right)$ with $-x \prec+\phi_{-}$. Similarly, each $\psi$ in $\vec{\psi}$ is either of the form $\psi_{+}\left(p_{1} / ! x, \overrightarrow{p^{\prime}} / \vec{z}\right)$ with $+x \prec-\psi_{+}$, or of the form $\psi_{-}\left(p_{2} / ! x, \overrightarrow{p^{\prime}} / \bar{z}\right)$ with $-x \prec-\psi_{-}$. Recall that each $\phi$ in $\vec{\phi}$ is definite positive PIA and each $\psi$ in $\vec{\psi}$ is definite negative PIA. Hence, we can assume w.l.o.g. that there are no occurrences of $-\perp$ and $+\top$ in each $+\phi$. Indeed, otherwise, the exhaustive application of the additional rules which identify $-f^{\prime}\left(\phi_{1}, \ldots, \perp^{\varepsilon_{f^{\prime}}}(i), \ldots, \phi_{n_{f^{\prime}}}\right)$ with $-\perp$ for every $f^{\prime} \in \mathcal{F} \cup\{\wedge\}$ and $+g^{\prime}\left(\phi_{1}, \ldots, \top^{\varepsilon_{g^{\prime}}}(i), \ldots, \phi_{n_{g^{\prime}}}\right)$ with $+\top$ for every $g^{\prime} \in \mathcal{G} \cup\{\vee\}$, would reduce all offending inequalities to tautological inequalities of the form $q \leq \top$ or $\perp \leq q$ which can then be removed. Likewise, we can assume w.l.o.g. that there are no occurrences of $+\perp$ and $-\top$ in each $+\psi$. This shows (cf. Remark 29) that we can assume w.l.o.g. that each $\phi$ in $\vec{\phi}$ (resp. $\psi$ in $\vec{\psi}$ ) is a right-primitive (resp. left-primitive) term. Likewise, we can assume w.l.o.g. that each $\gamma$ in $\vec{\gamma}$ (resp. $\chi$ in $\vec{\chi}$ ) is right-primitive (resp. leftprimitive). By Lemma 49 , the suitable derived adjunction rule among $\operatorname{LA}\left(\phi_{+}\right), \operatorname{LA}\left(\phi_{-}\right), \operatorname{RA}\left(\psi_{+}\right)$, $\mathrm{RA}\left(\psi_{-}\right)$is applicable to each $\phi$ and $\psi$, yielding:

$$
\begin{aligned}
& \forall \vec{p} \forall \vec{q}\left[\left(\vec{q}_{1,6} \leq \vec{\phi} \& \vec{\psi} \leq \vec{q}_{2,5} \& \vec{q}_{3,8} \leq \vec{\gamma} \& \vec{\chi} \leq \vec{q}_{4,7}\right) \Rightarrow \xi_{1}\left(\vec{q}_{1}, \vec{q}_{2}, \vec{q}_{3}, \vec{q}_{4}\right) \leq \xi_{2}\left(\vec{q}_{5}, \vec{q}_{6}, \vec{q}_{7}, \vec{q}_{8}\right)\right] \\
\text { iff } \quad & \forall \overrightarrow{p_{1}} \forall \overrightarrow{p_{2}} \forall \vec{q}\left[\left(\overrightarrow{\operatorname{LA}\left(\phi_{+}\right)(q / u)} \leq \vec{p}_{1} \& \overrightarrow{p_{2}} \leq \overrightarrow{\operatorname{LA}\left(\phi_{-}\right)(q / u)} \& \overrightarrow{\operatorname{RA}\left(\psi_{+}\right)(q / u) \leq \vec{p}_{1} \&}\right.\right. \\
& \left.\left.\overrightarrow{p_{2}} \leq \overrightarrow{\operatorname{RA}\left(\psi_{-}\right)(q / u)} \& \vec{q}_{3,8} \leq \vec{\gamma} \& \vec{\chi} \leq \vec{q}_{4,7}\right) \Rightarrow \xi_{1}\left(\vec{q}_{1}, \vec{q}_{2}, \vec{q}_{3}, \vec{q}_{4}\right) \leq \xi_{2}\left(\vec{q}_{5}, \vec{q}_{6}, \vec{q}_{7}, \vec{q}_{8}\right)\right] .
\end{aligned}
$$

Notice that, when applying the adjunction/residuation rules, the polarity of subterms which are parametric in the rule application remains unchanged. Hence, the assumption that there are no occurrences of $-\perp$ and $+\top$ in each $+\phi$ and there are no occurrences of $+\perp$ and $-\top$ in each $+\psi$ implies that there are no occurrences of $-\perp$ and $+\top$ in each $+\operatorname{LA}\left(\phi_{-}\right)(q / u)$ and $+\operatorname{RA}\left(\psi_{-}\right)(q / u)$, which are then shown to be right-primitive, and there are no occurrences of $+\perp$ and $-\top$ in each $+\operatorname{LA}\left(\phi_{+}\right)(q / u)$ and $+\operatorname{RA}\left(\psi_{+}\right)(q / u)$, which are then shown to be left-primitive. The assumptions made above imply that each $\gamma$ is antitone in each variable in $\vec{p}_{1}$ and monotone in each variable in $\overrightarrow{p_{2}}$, while each $\chi$ is monotone in each variable in $\overrightarrow{p_{1}}$ and antitone in each variable in $\overrightarrow{p_{2}}$. Hence, the quasi-inequality above is simultaneously in Ackermann shape w.r.t. all variables in $\vec{p} .{ }^{41}$ Applying the Ackermann rule repeatedly in the order indicated by $\Omega$ yields the following quasi-inequality, free of

\footnotetext{
${ }^{41}$ The formulas $\operatorname{LA}\left(\phi_{+}\right)(q / u), \operatorname{LA}\left(\phi_{-}\right)(q / u), \operatorname{RA}\left(\psi_{+}\right)(q / u)$ and $\operatorname{RA}\left(\psi_{-}\right)(q / u)$ do not need to be free of all variables in $\vec{p}$, and in general they are not. However, the assumptions and the general theory of ALBA guarantee that they are $\varepsilon^{\partial}$-uniform and free of the specific $p$-variable the 'minimal valuation' of which they are part of. The reader is referred to [18] for an expanded treatment of this point.
} 
variables in $\vec{p}$ :

$$
\forall \vec{q}\left[\left(\vec{q}_{3,8} \leq \vec{\gamma}\left(\vec{P}_{1} / \vec{p}_{1}, \vec{P}_{2} / \vec{p}_{2}\right) \& \vec{\chi}\left(\vec{P}_{1} / \vec{p}_{1}, \vec{P}_{2} / \vec{p}_{2}\right) \leq \vec{q}_{4,7}\right) \Rightarrow \xi_{1}\left(\vec{q}_{1}, \vec{q}_{2}, \vec{q}_{3}, \vec{q}_{4}\right) \leq \xi_{2}\left(\vec{q}_{5}, \vec{q}_{6}, \vec{q}_{7}, \vec{q}_{8}\right)\right]
$$

where $P_{1}$ and $P_{2}$ denote the $\mathcal{L}_{\text {DLE }}^{*}$-terms, with variables in $\vec{q}_{1}, \vec{q}_{2}, \vec{q}_{5}, \vec{q}_{6}$, obtained by applying the Ackermann substitution. For instance, for every $\Omega$-minimal $p_{1}$ in $\overrightarrow{p_{1}}$,

$$
P_{1}:=\bigvee_{i} \operatorname{LA}\left(\phi_{+}^{(i)}\right)(q / u) \vee \bigvee_{j} \operatorname{RA}\left(\psi_{+}^{(j)}\right)(q / u),
$$

and for every $\Omega$-minimal $p_{2}$ in $\overrightarrow{p_{2}}$,

$$
P_{2}:=\bigwedge_{i} \operatorname{LA}\left(\phi_{-}^{(i)}\right)(q / u) \wedge \bigwedge_{j} \operatorname{RA}\left(\psi_{-}^{(j)}\right)(q / u) .
$$

In the clauses above, the indexes $i$ and $j$ count the number of critical occurrences of the given variable $p_{1}$ (resp. $p_{2}$ ) in PIA-subterms of type $\phi_{+}$and $\psi_{+}$(resp. $\phi_{-}$and $\psi_{-}$).

Let us show that the quasi-inequality (7.1) represents the validity in perfect DLEs of some analytic (in fact quasi-special, cf. Definition 8) structural rule of the calculus DL. We have already observed above that $\xi_{1}$ is left-primitive and $\xi_{2}$ is right-primitive. Hence, the conclusion of the quasi-inequality (7.1) can be understood as the semantic interpretation of some structural sequent (cf. Definition 31). To see that each inequality in the antecedent of (7.1) is also the interpretation of some structural sequent, it is enough to show that every $\gamma\left(\vec{P}_{1} / \vec{p}_{1}, \vec{P}_{2} / \vec{p}_{2}\right)$ is right-primitive, and every $\chi\left(\vec{P}_{1} / \vec{p}_{1}, \vec{P}_{2} / \vec{p}_{2}\right)$ is leftprimitive. Indeed, if this is the case, then we can apply distribution rules exhaustively so as to surface the $+\vee$ and $-\wedge$, and then apply splitting rules to obtain definite left-primitive and right-primitive inequalities. By Definition 31 each of these inequalities will be the interpretation of some structural sequent.

This is a rather simple proof by induction on the maximum length of chains in $\Omega$. The base case, when $\Omega$ is the discrete order (hence $P_{1}$ and $P_{2}$ are of the form displayed above), immediately follows from the observation, made above, that each $\gamma$ is right-primitive, antitone in each variable in $\overrightarrow{p_{1}}$ and monotone in each variable $\overrightarrow{p_{2}}$, while each $\chi$ is left-primitive, monotone in each variable in $\overrightarrow{p_{1}}$ and antitone in each variable $\overrightarrow{p_{2}}$, and by Lemma 49 . The inductive step is routine.

Let us show that the rule so obtained is analytic (cf. Definition 4), i.e. it satisfies conditions $\mathrm{C}_{1}-\mathrm{C}_{7}$. As to $\mathrm{C}_{1}$, notice that each variable $q$ in $\vec{q}_{i}$ for $1 \leq i \leq 8$ appears in some inequality in the antecedent of the initial quasi-inequality, and has not been eliminated in any ensuing transformations. This implies that each $q$ gives rise to a parametric structural variable $X$ which occurs in some premise and in the conclusion. Condition $\mathrm{C}_{2}$ is guaranteed by construction: indeed, the congruence relation is defined as the transitive closure of the relation identifying only the occurrences of the structural variable $X$ corresponding to one variable $q$. Condition $\mathrm{C}_{3}$ is also guaranteed by construction, given that each variable $q$ occurs exactly once in $\xi_{1}\left(\vec{q}_{1}, \vec{q}_{2}, \vec{q}_{3}, \vec{q}_{4}\right) \leq \xi_{2}\left(\vec{q}_{5}, \vec{q}_{6}, \vec{q}_{7}, \vec{q}_{8}\right)$. Condition $\mathrm{C}_{4}$ follows from Remark 58, and the fact that adjunction rules and usual Ackermann rule preserve the polarity of the variables. Condition $\mathrm{C}_{5}$ vacuously holds, since all constituents of structural rules are parametric. Conditions $\mathrm{C}_{6}$ and $\mathrm{C}_{7}$ are immediate.

Finally, observe that the rule we have obtained is in fact quasi-special. Indeed, the variables $\vec{q}_{3}, \vec{q}_{4}, \vec{q}_{7}, \vec{q}_{8}$ are fresh, and each of them occurs only once in the premises. 


\subsection{From analytic rules to analytic inductive inequalities}

In the previous section, we introduced the syntactic shape of analytic inductive $\mathcal{L}_{\mathrm{DLE}}$-inequalities for any language $\mathcal{L}_{\text {DLE }}$, and showed that these inequalities can be effectively transformed via ALBA into a set of analytic structural rules of the associated display calculus DL $\mathbf{L}_{\mathrm{DLE}}$. In the present section, we show that having this shape is also a necessary condition.

LEMMA 60

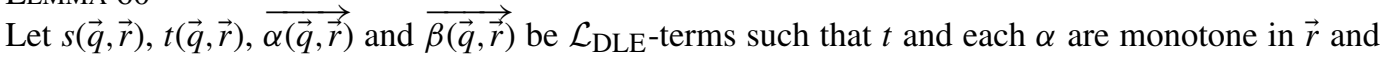
antitone in $\vec{q}$, and $s$ and each $\beta$ are monotone in $\vec{q}$ and antitone in $\vec{r}$. Then the following are equivalent for any DLE $\mathbb{A}$ :

(1) $\mathbb{A} \models s(\vec{q} \wedge \vec{\alpha} / \vec{q}, \vec{r} \vee \vec{\beta} / \vec{r}) \leq t(\vec{q} \wedge \vec{\alpha} / \vec{q}, \vec{r} \vee \vec{\beta} / \vec{r})$;

(2) $\mathbb{A} \models \forall \vec{q} \forall \vec{r}[(\vec{q} \leq \vec{\alpha} \& \vec{\beta} \leq \vec{r}) \Rightarrow s(\vec{q}, \vec{r}) \leq t(\vec{q}, \vec{r})]$.

Proof. Assume item (1). To show item (2), fix a valuation $v$ such that $(\mathbb{A}, v) \models \vec{q} \leq \vec{\alpha}$ and $(\mathbb{A}, v) \models$ $\vec{\beta} \leq \vec{r}$. Hence, $(\mathbb{A}, v) \models \vec{q} \wedge \vec{\alpha}=\vec{q}$ and $(\mathbb{A}, v) \models \vec{r} \vee \vec{\beta}=\vec{r}$. By item $(1),(\mathbb{A}, v) \models s(\vec{q} \wedge \vec{\alpha} / \vec{q}, \vec{r} \vee \vec{\beta} / \vec{r}) \leq t(\vec{q} \wedge$ $\vec{\alpha} / \vec{q}, \vec{r} \vee \vec{\beta} / \vec{r})$, which is equivalent to $(\mathbb{A}, v)=s(\vec{q}, \vec{r}) \leq t(\vec{q}, \vec{r})$, as required.

Conversely, assume item (2) and fix a valuation $v$. Clearly, $(\mathbb{A}, v) \models \vec{q} \wedge \vec{\alpha} \leq \vec{\alpha}$ and $(\mathbb{A}, v) \models \vec{\beta} \leq \vec{r} \vee \vec{\beta}$. Since each $\alpha$ (resp. $\beta$ ) is monotone (resp. antitone) in $\vec{r}$ and antitone (resp. monotone) in $\vec{q}$, this implies that

$$
(\mathbb{A}, v) \models \overrightarrow{\alpha(\vec{q}, \vec{r})} \leq \overrightarrow{\alpha((\vec{q} \wedge \vec{\alpha}) / \vec{q},(\vec{r} \vee \vec{\beta}) / \vec{r})} \quad(\mathbb{A}, v) \models \overrightarrow{\beta((\vec{q} \wedge \vec{\alpha}) / \vec{q},(\vec{r} \vee \vec{\beta}) / \vec{r})} \leq \overrightarrow{\beta(\vec{q}, \vec{r})},
$$

which immediately entail that

$$
(\mathbb{A}, v)=\vec{q} \wedge \overrightarrow{\alpha(\vec{q}, \vec{r})} \leq \overrightarrow{\alpha((\vec{q} \wedge \vec{\alpha}) / \vec{q},(\vec{r} \vee \vec{\beta}) / \vec{r})} \quad(\mathbb{A}, v)=\overrightarrow{\beta((\vec{q} \wedge \vec{\alpha}) / \vec{q},(\vec{r} \vee \vec{\beta}) / \vec{r})} \leq \overrightarrow{\beta(\vec{q}, \vec{r}) \vee \vec{r}}
$$

Let $v^{\prime}$ be the $\vec{q} \oplus \vec{r}$-variant of $v$ such that $\overrightarrow{v^{\prime}(q)}:=\overrightarrow{v(q)} \wedge \overrightarrow{\llbracket \alpha \rrbracket_{v}}$ and $\overrightarrow{v^{\prime}(r)}:=\overrightarrow{v(r)} \vee \overrightarrow{\llbracket \beta \rrbracket_{v}}$. By definition, the conditions above are equivalent to

$$
\left(\mathbb{A}, v^{\prime}\right) \models \vec{q} \leq \overrightarrow{\alpha(\vec{q}, \vec{r})} \quad\left(\mathbb{A}, v^{\prime}\right) \models \overrightarrow{\beta(\vec{q}, \vec{r})} \leq \vec{r} .
$$

Hence, by assumption 2 , we can conclude that $\left(\mathbb{A}, v^{\prime}\right) \models s \leq t$, which is equivalent to $(\mathbb{A}, v) \models s(\vec{q} \wedge$ $\vec{\alpha} / \vec{q}, \vec{r} \vee \vec{\beta} / \vec{r}) \leq t(\vec{q} \wedge \vec{\alpha} / \vec{q}, \vec{r} \vee \vec{\beta} / \vec{r})$, as required.

PROPOSITION 61

For any language $\mathcal{L}_{\mathrm{DLE}}$, every analytic rule in the language of the corresponding calculus DL is semantically equivalent to some analytic inductive $\mathcal{L}_{\text {DLE }}^{*}$-inequality.

PROOF. Modulo application of display postulates, any analytic rule can be represented as follows:

$$
\left(S_{j}^{i} \vdash Y^{i} \mid 1 \leq i \leq n \text { and } 1 \leq j \leq n_{i}\right) \quad\left(X^{k} \vdash T_{\ell}^{k} \mid 1 \leq k \leq m \text { and } 1 \leq \ell \leq m_{k}\right)
$$

$$
(S \vdash T)\left[Y^{i}\right]^{\text {suc }}\left[X^{k}\right]^{\text {pre }}
$$

where $Y^{i}$ and $X^{k}$ are structural variables and $S_{j}^{i}, T_{\ell}^{k}, S$ and $T$ are structural terms. As discussed in Section 4.2 , for every perfect $\mathcal{L}_{\mathrm{DLE}}$-algebra $\mathbb{A}$, the validity of the rule above on $\mathbb{A}$ is equivalent to the validity on $\mathbb{A}$ of the following quasi-inequality

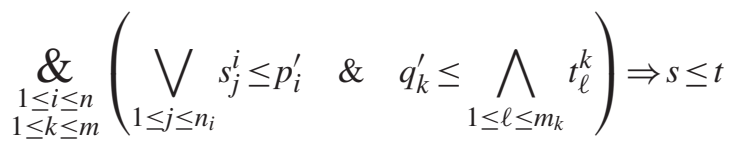


where $p_{i}^{\prime}:=r\left(Y^{i}\right), q_{k}^{\prime}:=l\left(X^{k}\right)$, for each $i$ and $k$, and $s:=l(S), t:=r(T)$, and $s_{j}^{i}:=l\left(S_{j}^{i}\right)$, and $t_{\ell}^{k}:=r\left(T_{\ell}^{k}\right)$ for each $j$ and $\ell$. Let $s_{i}:=\bigvee_{1 \leq j \leq n_{i}} s_{j}^{i}$ and $t_{k}:=\bigwedge_{1 \leq \ell \leq m_{k}} t_{\ell}^{k}$.

By Lemma 60, the validity of the quasi-inequality above is equivalent to the validity of the following inequality, where $\overrightarrow{p^{\prime}}:=\left(p_{i}^{\prime}\right)_{i}, \vec{s}:=\left(s_{i}\right)_{i}, \overrightarrow{q^{\prime}}:=\left(q_{k}^{\prime}\right)_{k}$ and $\vec{t}:=\left(t_{k}\right)_{k}$ :

$$
s\left(\left(\overrightarrow{p^{\prime}} \vee \vec{s}\right) / \overrightarrow{p^{\prime}},\left(\overrightarrow{q^{\prime}} \wedge \vec{t}\right) / \overrightarrow{q^{\prime}}\right) \leq t\left(\left(\overrightarrow{p^{\prime}} \vee \vec{s}\right) / \overrightarrow{p^{\prime}},\left(\overrightarrow{q^{\prime}} \wedge \vec{t}\right) / \overrightarrow{q^{\prime}}\right)
$$

To finish the proof, we need to show that the inequality above is analytic $(\Omega, \varepsilon)$-inductive for some $\Omega$ and $\varepsilon$. Let $\vec{p}, \vec{q}$ be the variables in the inequality $s \leq t$, different from the variables in $\vec{p}^{\prime}$ and $\overrightarrow{q^{\prime}}$, and occurring in $s \vdash t$ in antecedent and succedent position, respectively (by $\mathrm{C}_{4}$ they are disjoint). Clearly, $s\left(\vec{p}, \vec{q}, \overrightarrow{p^{\prime}}, \overrightarrow{q^{\prime}}\right)$ is left-primitive, and hence is positive skeleton, and $t\left(\vec{p}, \vec{q}, \overrightarrow{p^{\prime}}, \overrightarrow{q^{\prime}}\right)$ is right-primitive, and hence is negative skeleton. Condition $\mathrm{C}_{3}$ implies that $X^{k}$ and $Y^{i}$ are in antecedent and succedent position, respectively, in $S \vdash T$, and hence $s$ (resp. $t$ ) is monotone in $\vec{q}^{\prime}$ (resp. in $\vec{p}^{\prime}$ ) and antitone in $\overrightarrow{p^{\prime}}$ (resp. $\overrightarrow{q^{\prime}}$ ). Moreover, $s_{i}$ is left-primitive, and hence is negative PIA for every $i$, and $t_{k}$ is rightprimitive, and hence is positive PIA for every $k$. These observations immediately yield that every branch in the inequality (7.2) is good, and in particular, $\vec{s}$ and $\vec{t}$ are the PIA-parts.

Next, let $\varepsilon$ be the order type which assigns all $p$ in $\vec{p}$ and $q^{\prime}$ in $\overrightarrow{q^{\prime}}$ to 1 and all $q$ in $\vec{q}$ and $q$ in $\overrightarrow{p^{\prime}}$ to $\partial$. Let $\Omega$ be the discrete order. To show that the inequality (7.2) is analytic $(\Omega, \varepsilon)$-inductive, it is enough to show that all terms in $\vec{s}$ and $\vec{t}$ are $\varepsilon^{\partial}$-uniform.

Since any $p$ in $\vec{p}$ corresponds to a structural variable antecedent position, $+p \prec+s_{i}$ and $+p \prec-t_{k}$ for all $i$ and $k$, hence $-p \prec-s_{i}$ and $-p \prec+t_{k}$ for all $i$ and $k$. This shows that $\vec{s}$ and $\vec{t}$ are $\varepsilon^{\partial}$-uniform in any $p$ in $\vec{p}$. Similar arguments relative to the variables in $\vec{q}, \overrightarrow{p^{\prime}}$ and $\overrightarrow{q^{\prime}}$ complete the proof.

REMARK 62

If the rule

$$
\frac{\left(S_{j}^{i} \vdash Y^{i} \mid 1 \leq i \leq n \text { and } 1 \leq j \leq n_{i}\right) \quad\left(X^{k} \vdash T_{\ell}^{k} \mid 1 \leq k \leq m \text { and } 1 \leq \ell \leq m_{k}\right)}{(S \vdash T)\left[Y^{i}\right]^{\text {suc }}\left[X^{k}\right]^{\text {pre }}}
$$

is quasi-special, then, in order to transform it into an analytic inequality as in the proof of the proposition above, we can use Lemma 57 rather than Lemma 60, which yields the inequality

$$
s\left(\vec{s} / \overrightarrow{p^{\prime}}, \vec{t} / \overrightarrow{q^{\prime}}\right) \leq t\left(\vec{s} / \overrightarrow{p^{\prime}}, \vec{t} / \overrightarrow{q^{\prime}}\right)
$$

which is equivalent to (7.2). Indeed, all variables in $\overrightarrow{p^{\prime}}$ occur only in positive position and can hence be equivalently replaced by $\perp$ and all variables in $\overrightarrow{q^{\prime}}$ occur only in negative position and can be equivalently replaced by $T$, yielding

$$
s\left((\vec{\perp} \vee \vec{s}) / \vec{p}^{\prime},(\vec{\top} \wedge \vec{t}) / \vec{q}^{\prime}\right) \leq t\left((\vec{\perp} \vee \vec{s}) / \overrightarrow{p^{\prime}},(\vec{\top} \wedge \vec{t}) / \vec{q}^{\prime}\right),
$$

which is equivalent to (7.3). We will come back to this observation in the following section.

\section{Special rules are as expressive as analytic rules}

In [38], Kracht states without proof that every analytic rule in the display calculus for the classical basic tense logic $K t$ is equivalent to a special rule (see also the discussion in [10, Section 5.1]). A proof of this fact is presented in [10], where it is shown that, in classical tense logic, every axiom which is obtained from an analytic rule of the display calculus is equivalent to a primitive axiom. 
In the present section, we extend this result from classical tense logic to any DLE-logic. Namely, we show, using ALBA, that every analytic inductive inequality in any DLE-language is equivalent to some primitive inequality in the corresponding DLE*-language. We will proceed in two steps: in Section 8.1, we will present an intermediate subclass of analytic inductive inequalities, referred to as quasi-primitive inequalities, and show that any analytic inductive inequality can be equivalently transformed into some quasi-primitive inequality. Then, in Section 8.2, we will prove that every quasi-primitive inequality is equivalent to some primitive inequality.

These results imply that special structural rules (cf. Definition 6) are as expressive as analytic rules (cf. Definition 4). Hence, for any language $\mathcal{L}_{\text {DLE }}$, any properly displayable DLE-logic is specially displayable. Notice that this fact does not imply that any properly displayable $\mathcal{L}_{\text {DLE}}$-logic can be axiomatized by means of primitive $\mathcal{L}_{\mathrm{DLE}}$-inequalities, since the required primitive inequalities pertain to the language $\mathcal{L}_{\mathrm{DLE}}^{*}$. However, this fact does imply that any properly displayable $\mathcal{L}_{\text {DLE }}^{*}$-logic can be axiomatized by means of primitive $\mathcal{L}_{\mathrm{DLE}}^{*}$-inequalities.

\subsection{Quasi-special rules and quasi-special inductive inequalities}

Let us take stock of what was presented in Sections 7.1 and 7.2. Taken together, Propositions 61 and 59 immediately imply that every analytic rule is equivalent to a quasi-special rule. Furthermore, any analytic inductive inequality derived from an analytic rule has a special shape: every critical branch consists only of Skeleton nodes, leaving all PIA subtrees to be $\varepsilon^{\partial}$-uniform. This motivates the following definition:

\section{DeFinition 63}

For every analytic $(\Omega, \varepsilon)$-inductive inequality $s \leq t$, if every $\varepsilon$-critical branch of the signed generation trees $+s$ and $-t$ consists solely of skeleton nodes, then $s \leq t$ is a quasi-special inductive inequality. Such an inequality is definite if none of its Skeleton nodes is $+\vee$ or $-\wedge$.
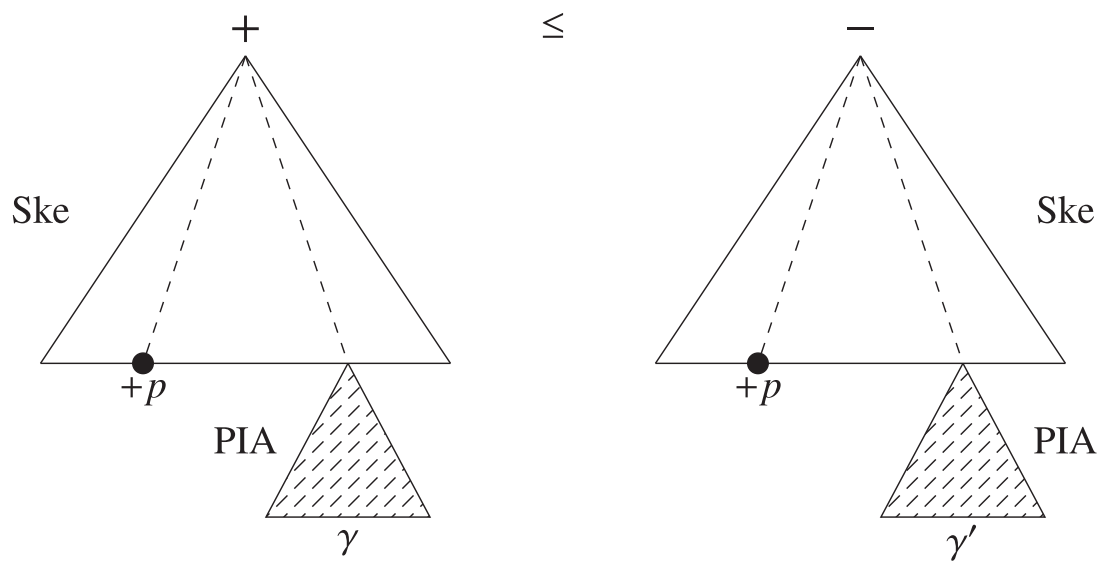

Definite quasi-special inductive inequalities and quasi-special rules entertain the same privileged relation with each other as the one entertained by definite primitive inequalities and special rules. Indeed, translating into an inequality the rule obtained from a definite quasi-special inductive inequality leads to the original inequality (cf. Remark 62). Notice that these are exactly the inequalities that have this property, since the inequality that is obtained by Proposition 61 is always definite quasi-special inductive. Since every analytic inductive inequality is equivalent to a set of analytic rules (in fact quasi-special rules) and every analytic rule is equivalent to a definite quasi-special 
inductive inequality, is it clear that every analytic inductive inequality is equivalent to a set of definite quasi-special inductive inequalities.

\subsection{Quasi-special inductive inequalities are equivalent to primitive inequalities}

\section{PROPOSITION 64}

Let $\xi_{1}(! \vec{x}, ! \vec{y}, ! \vec{z}, ! \vec{w})$ be a definite positive Skeleton formula and $\xi_{2}(\vec{x}, \vec{y})$ be a positive Skeleton formula such that $+\vec{x},+\vec{z} \prec+\xi_{1},-\vec{y},-\vec{w} \prec+\xi_{1}$ and $-\vec{x},+\vec{y} \prec-\xi_{2}$. Let $\overrightarrow{\gamma(\vec{p}, \vec{q})}$ be an array of positive PIAformulas such that $-\vec{p},+\vec{q} \prec+\gamma$ and let $\overrightarrow{\chi(\vec{p}, \vec{q})}$ be an array of negative PIA formulas such that $-\vec{p},+\vec{q} \prec-\chi$. Then the following are equivalent:

(1) $\forall \vec{p} \forall \vec{q}\left[\xi_{1}(\vec{p} / \vec{x}, \vec{q} / \vec{y}, \vec{\gamma} / \vec{z}, \vec{\chi} / \vec{w}) \leq \xi_{2}(\vec{p} / \vec{x}, \vec{q} / \vec{y})\right]$;

(2)

$$
\forall \vec{p} \forall \vec{q} \forall \overrightarrow{p^{\prime}} \forall \overrightarrow{q^{\prime}}\left[\begin{array}{rl}
\xi_{1}\left(\vec{p} / \vec{x}, \vec{q} / \vec{y}, \vec{p}^{\prime} / \vec{z}, \overrightarrow{q^{\prime}} / \vec{w}\right) \leq & \xi_{2}(\vec{p} / \vec{x}, \vec{q} / \vec{y}) \vee \\
& \left(\bigvee_{z_{k} \in \vec{z}} \xi_{1}\left(\vec{p} / \vec{x}, \vec{q} / \vec{y}, \overrightarrow{p^{\prime}}-k / \vec{z}-k, p_{k}^{\prime}-\gamma_{k} / z_{k}, \overrightarrow{q^{\prime}} / \vec{w}\right)\right) \vee \\
& \left(\bigvee_{w_{\ell} \in \vec{w}} \xi_{1}\left(\vec{p} / \vec{x}, \vec{q} / \vec{y}, \vec{p}^{\prime} / \vec{z}, \vec{q}^{\prime}-\ell / \vec{w}-\ell, \chi_{\ell} \rightarrow q_{\ell}^{\prime} / w_{\ell}\right)\right)
\end{array}\right] .
$$

Proof. The inequality in item (1) of the statement can be equivalently transformed via ALBA into the following quasi-inequality:

$$
\forall \mathbf{i} \forall \overrightarrow{\mathbf{j}} \forall \overrightarrow{\mathbf{j}^{\prime}} \forall \overrightarrow{\mathbf{n}} \forall \overrightarrow{\mathbf{n}^{\prime}} \forall \mathbf{m}\left[\left(\overrightarrow{\mathbf{j}^{\prime}} \leq \overrightarrow{\gamma(\overrightarrow{\mathbf{j}}, \overrightarrow{\mathbf{n}})} \& \overrightarrow{\chi(\overrightarrow{\mathbf{j}}, \overrightarrow{\mathbf{n}})} \leq \overrightarrow{\mathbf{n}^{\prime}} \& \xi_{2}(\overrightarrow{\mathbf{j}}, \overrightarrow{\mathbf{n}}) \leq \mathbf{m}\right) \Rightarrow \xi_{1}\left(\overrightarrow{\mathbf{j}}, \overrightarrow{\mathbf{n}}, \overrightarrow{\mathbf{j}^{\prime}}, \overrightarrow{\mathbf{n}^{\prime}}\right) \leq \mathbf{m}\right]
$$

Likewise, the inequality in item (2) can be equivalently transformed via ALBA into the following quasi-inequality:

$$
\forall \mathbf{i} \forall \overrightarrow{\mathbf{j}} \forall \overrightarrow{\mathbf{j}^{\prime}} \forall \overrightarrow{\mathbf{n}} \forall \overrightarrow{\mathbf{n}^{\prime}} \forall \mathbf{m}\left[\left(\begin{array}{c}
\&_{k} \xi_{1}\left(\overrightarrow{\mathbf{j}}, \overrightarrow{\mathbf{n}}, \overrightarrow{\mathbf{j}}^{\prime}-k, \mathbf{j}_{k}^{\prime}<\gamma_{k}(\overrightarrow{\mathbf{j}}, \overrightarrow{\mathbf{n}}), \overrightarrow{\mathbf{n}}^{\prime}\right) \leq \mathbf{m} \\
\&_{\ell} \xi_{1}\left(\overrightarrow{\mathbf{j}}, \overrightarrow{\mathbf{n}}, \overrightarrow{\mathbf{j}^{\prime}}, \overrightarrow{\mathbf{n}^{\prime}}-\ell, \chi_{\ell}(\overrightarrow{\mathbf{j}}, \overrightarrow{\mathbf{n}}) \rightarrow \mathbf{n}_{\ell}^{\prime}\right) \leq \mathbf{m} \\
\xi_{2}(\overrightarrow{\mathbf{j}}, \overrightarrow{\mathbf{n}}) \leq \mathbf{m}
\end{array}\right) \Rightarrow \xi_{1}\left(\overrightarrow{\mathbf{j}}, \overrightarrow{\mathbf{n}}, \overrightarrow{\mathbf{j}^{\prime}}, \overrightarrow{\mathbf{n}^{\prime}}\right) \leq \mathbf{m}\right]
$$

To finish the proof, it is enough to show that conditions (8.1) and (8.2) are equivalent. Assume condition (8.2) and let $\overrightarrow{\mathbf{j}} \overrightarrow{\mathbf{n}}, \mathbf{m}, \mathbf{j}_{k}^{\prime}$ and $\mathbf{n}_{\ell}^{\prime}$ be such that the following inequalities hold for any $\gamma_{k}$ in $\vec{\gamma}$ and $\chi_{\ell}$ in $\vec{\chi}$ :

$$
\mathbf{j}_{k}^{\prime} \leq \gamma_{k}(\overrightarrow{\mathbf{j}}, \overrightarrow{\mathbf{n}}) \quad \chi_{\ell}(\overrightarrow{\mathbf{j}}, \overrightarrow{\mathbf{n}}) \leq \mathbf{n}_{\ell}^{\prime} \quad \xi_{2}(\overrightarrow{\mathbf{j}}, \overrightarrow{\mathbf{n}}) \leq \mathbf{m} .
$$

By applying adjunction all inequalities above but the last one become

$$
\mathbf{j}_{k}^{\prime}<\gamma_{k}(\overrightarrow{\mathbf{j}}, \overrightarrow{\mathbf{n}})=\perp \quad \top=\chi_{\ell}(\overrightarrow{\mathbf{j}}, \overrightarrow{\mathbf{n}}) \rightarrow \mathbf{n}_{\ell}^{\prime} .
$$

These equalities imply that

$$
\&_{k} \xi_{1}\left(\overrightarrow{\mathbf{j}}, \overrightarrow{\mathbf{n}}, \overrightarrow{\mathbf{j}}^{\prime}{ }_{k}, \mathbf{j}_{k}^{\prime}<\gamma_{k}(\overrightarrow{\mathbf{j}}, \overrightarrow{\mathbf{n}}), \overrightarrow{\mathbf{n}}^{\prime}\right)=\perp \quad \&_{\ell} \xi_{1}\left(\overrightarrow{\mathbf{j}}, \overrightarrow{\mathbf{n}}, \overrightarrow{\mathbf{j}^{\prime}}, \overrightarrow{\mathbf{n}}^{\prime}-\ell, \chi_{\ell}(\overrightarrow{\mathbf{j}}, \overrightarrow{\mathbf{n}}) \rightarrow \mathbf{n}_{\ell}^{\prime}\right)=\perp .
$$

Indeed, by assumption, $\xi_{1}$ is a definite positive Skeleton formula such that any variable in it occurs at most once. Hence, $\xi_{1}$ is a definite and scattered left-primitive formula. By Lemma 35, the term function induced by $\xi_{1}$ is an operator, and hence $\xi_{1}$ preserves $\perp$ in its positive coordinates and reverses 
$\top$ in its negative coordinates. This finishes the proof that all $\overrightarrow{\mathbf{j}} \overrightarrow{\mathbf{n}}, \mathbf{m}, \mathbf{j}_{k}^{\prime}$ and $\mathbf{n}_{\ell}^{\prime}$ satisfying conditions (8.3) satisfy also the premises of the quasi-inequality (8.2), namely:

$$
\&_{k} \xi_{1}\left(\overrightarrow{\mathbf{j}}, \overrightarrow{\mathbf{n}}, \overrightarrow{\mathbf{j}}^{\prime}-k, \overrightarrow{\mathbf{j}}_{k}^{\prime}-\gamma_{k}(\overrightarrow{\mathbf{j}}, \overrightarrow{\mathbf{n}}), \overrightarrow{\mathbf{n}}^{\prime}\right) \leq \mathbf{m} \quad \&_{\ell} \xi_{1}\left(\overrightarrow{\mathbf{j}}, \overrightarrow{\mathbf{n}}, \overrightarrow{\mathbf{j}^{\prime}}, \overrightarrow{\mathbf{n}}^{\prime}-\ell, \chi_{\ell}(\overrightarrow{\mathbf{j}}, \overrightarrow{\mathbf{n}}) \rightarrow \mathbf{n}_{\ell}^{\prime}\right) \leq \mathbf{m} \quad \xi_{2}(\overrightarrow{\mathbf{j}}, \overrightarrow{\mathbf{n}}) \leq \mathbf{m} .
$$

By assumption (8.2), we conclude that $\xi_{1}\left(\overrightarrow{\mathbf{j}}, \overrightarrow{\mathbf{n}}, \overrightarrow{\mathbf{j}^{\prime}}, \overrightarrow{\mathbf{n}}^{\prime}\right) \leq \mathbf{m}$, as required.

Conversely, assume condition (8.1) and let $\overrightarrow{\mathbf{j}} \overrightarrow{\mathbf{n}}, \mathbf{m}, \mathbf{j}_{k}^{\prime}$ and $\mathbf{n}_{\ell}^{\prime}$ be such that the following inequalities hold for any $k$ and $\ell$ as above:

$$
\xi_{1}\left(\overrightarrow{\mathbf{j}}, \overrightarrow{\mathbf{n}}, \overrightarrow{\mathbf{j}}_{-k}^{\prime}, \mathbf{j}_{k}^{\prime}-\gamma_{k}(\overrightarrow{\mathbf{j}}, \overrightarrow{\mathbf{n}}), \overrightarrow{\mathbf{n}}^{\prime}\right) \leq \mathbf{m} \quad \xi_{1}\left(\overrightarrow{\mathbf{j}}, \overrightarrow{\mathbf{n}}, \overrightarrow{\mathbf{j}}^{\prime}, \overrightarrow{\mathbf{n}}_{-\ell}^{\prime}, \chi_{\ell}(\overrightarrow{\mathbf{j}}, \overrightarrow{\mathbf{n}}) \rightarrow \mathbf{n}_{\ell}^{\prime}\right) \leq \mathbf{m} \quad \xi_{2}(\overrightarrow{\mathbf{j}}, \overrightarrow{\mathbf{n}}) \leq \mathbf{m} .
$$

By applying the appropriate residuation rules, all but the last inequality above can be equivalently written as follows:

$$
\mathbf{j}_{k}^{\prime}-\gamma_{k}(\overrightarrow{\mathbf{j}}, \overrightarrow{\mathbf{n}}) \leq \operatorname{RA}\left(\xi_{1}\right)\left(\overrightarrow{\mathbf{j}}, \overrightarrow{\mathbf{n}}, \overrightarrow{\mathbf{j}}^{\prime}{ }_{k}, \mathbf{m} / u, \overrightarrow{\mathbf{n}}^{\prime}\right) \quad \operatorname{RA}\left(\xi_{1}\right)\left(\overrightarrow{\mathbf{j}}, \overrightarrow{\mathbf{n}}, \overrightarrow{\mathbf{j}^{\prime}}, \overrightarrow{\mathbf{n}}^{\prime}{ }_{\ell \ell}, \mathbf{m} / u\right) \leq \chi \ell(\overrightarrow{\mathbf{j}}, \overrightarrow{\mathbf{n}}) \rightarrow \mathbf{n}_{\ell}^{\prime}
$$

and by applying residuation once again we obtain for every $k$ and $\ell$ :

$$
\dot{\mathbf{j}}_{k}^{\prime} \leq \gamma_{k}(\overrightarrow{\mathbf{j}}, \overrightarrow{\mathbf{n}}) \vee \operatorname{RA}\left(\xi_{1}\right)\left(\overrightarrow{\mathbf{j}}, \overrightarrow{\mathbf{n}}, \overrightarrow{\mathbf{j}}_{-k}^{\prime}, \mathbf{m} / u, \overrightarrow{\mathbf{n}}^{\prime}\right) \quad \operatorname{RA}\left(\xi_{1}\right)\left(\overrightarrow{\mathbf{j}}, \overrightarrow{\mathbf{n}}, \overrightarrow{\mathbf{j}}^{\prime}, \overrightarrow{\mathbf{n}}_{-\ell}^{\prime}, \mathbf{m} / u\right) \wedge \chi_{\ell}(\overrightarrow{\mathbf{j}}, \overrightarrow{\mathbf{n}}) \leq \mathbf{n}_{\ell}^{\prime}
$$

Since each $\mathbf{j}_{k}^{\prime}$ and each $\mathbf{n}_{\ell}^{\prime}$ is a nominal and a conominal, respectively, they are interpreted as join-prime and meet-prime elements, respectively. If $\mathbf{j}_{k}^{\prime} \leq \gamma_{k}(\overrightarrow{\mathbf{j}}, \overrightarrow{\mathbf{n}})$ and $\chi_{\ell}(\overrightarrow{\mathbf{j}}, \overrightarrow{\mathbf{n}}) \leq \mathbf{n}_{\ell}^{\prime}$ for all $k$ and $\ell$, then the antecedent of $(8.1)$ is satisfied and hence we conclude $\xi_{1}\left(\overrightarrow{\mathbf{j}}, \overrightarrow{\mathbf{n}}, \overrightarrow{\mathbf{j}^{\prime}}, \overrightarrow{\mathbf{n}^{\prime}}\right) \leq \mathbf{m}$. If $\mathbf{j}_{k}^{\prime} \leq$ $\operatorname{RA}\left(\xi_{1}\right)\left(\overrightarrow{\mathbf{j}}, \overrightarrow{\mathbf{n}}, \overrightarrow{\mathbf{j}}^{\prime}-k, \mathbf{m} / u, \overrightarrow{\mathbf{n}}^{\prime}\right)$ or $\operatorname{RA}\left(\xi_{1}\right)\left(\overrightarrow{\mathbf{j}}, \overrightarrow{\mathbf{n}}, \overrightarrow{\mathbf{j}^{\prime}}, \overrightarrow{\mathbf{n}}^{\prime}{ }_{\ell}, \mathbf{m} / u\right) \leq \mathbf{n}_{\ell}^{\prime}$ for some $\mathbf{j}_{k}^{\prime}$ or $\mathbf{n}_{\ell}^{\prime}$, then by applying the appropriate residuation rule we immediately obtain that $\xi_{1}\left(\overrightarrow{\mathbf{j}}, \overrightarrow{\mathbf{n}}, \overrightarrow{\mathbf{j}^{\prime}}, \overrightarrow{\mathbf{n}^{\prime}}\right) \leq \mathbf{m}$.

The following proposition is order-dual to the previous one, hence its proof is omitted.

\section{PROPOSITION 65}

Let $\xi_{1}(\vec{x}, \vec{y})$ be a negative Skeleton formula and $\xi_{2}(! \vec{x}, ! \vec{y}, ! \vec{z}, ! \vec{w})$ be a definite negative Skeleton formula such that $-\vec{x},+\vec{y} \prec+\xi_{1},+\vec{x},+\vec{z} \prec-\xi_{2}$ and $-\vec{y},-\vec{w} \prec-\xi_{2}$. Let $\overrightarrow{\gamma(\vec{p}, \vec{q})}$ be an array of positive PIAformulas such that $-\vec{p},+\vec{q} \prec+\gamma$ and let $\overrightarrow{\chi(\vec{p}, \vec{q})}$ be an array of negative PIA formulas such that $-\vec{p},+\vec{q} \prec-\chi$. Then the following are equivalent:

(1) $\forall \vec{p} \forall \vec{q}\left[\xi_{1}(\vec{p} / \vec{x}, \vec{q} / \vec{y}) \leq \xi_{2}(\vec{p} / \vec{x}, \vec{q} / \vec{y}, \vec{\gamma} / \vec{z}, \vec{\chi} / \vec{w})\right]$

(2)

$$
\forall \vec{p} \forall \vec{q} \forall \overrightarrow{p^{\prime}} \forall \overrightarrow{q^{\prime}}\left[\begin{array}{l}
\xi_{1}(\vec{p} / \vec{x}, \vec{q} / \vec{y}) \wedge \\
\left(\bigwedge_{z_{k} \in \vec{z}} \xi_{2}\left(\vec{p} / \vec{x}, \vec{q} / \vec{y}, \vec{p}^{\prime}-k / \vec{z}_{-k}, p_{k}^{\prime}-\gamma_{k} / z_{k}, \vec{q}^{\prime} / \vec{w}\right)\right) \wedge \\
\left(\bigwedge_{w_{\ell} \in \vec{w}} \xi_{2}\left(\vec{p} / \vec{x}, \vec{q} / \vec{y}, \vec{p}^{\prime} / \vec{z}, \vec{q}^{\prime}-\ell / \vec{w}-\ell, \chi_{\ell} \rightarrow q_{\ell}^{\prime} / w_{\ell}\right)\right) \leq \xi_{2}\left(\vec{p} / \vec{x}, \vec{q} / \vec{y}, \vec{p}^{\prime} / \vec{z}, \vec{q}^{\prime} / \vec{w}\right)
\end{array}\right] .
$$

\section{COROLlary 66}

For any language $\mathcal{L}_{\text {DLE }}$, every analytic structural rule in the language of the corresponding display calculus DL can be equivalently transformed into some special structural rule in the same language.

ProOF. As discussed at the beginning of Section 8.1, any analytic structural rule in DL is equivalent to a definite quasi-special inequality in $\mathcal{L}_{\mathrm{DLE}}^{*}$. It is easy to see that every definite quasi-special inequality can be transformed into one inequality of the form of item (1) in Proposition 64 or 65. This transformation is effected by applying suitable residuation rules so as to reduce one side of the given inequality to an $\varepsilon^{\partial}$-uniform PIA subterm (analogously to the treatment of the Type 4 inequalities 
discussed in Section 6.3). Hence, either Proposition 64 or 65 is applicable, yielding an equivalent inequality as in item (2) of the propositions mentioned above. Finally, the inequality in item (2) of Proposition 64 (resp. 65) is definite left-primitive (resp. right-primitive). Hence, the statement follows by Lemma 32.

\section{Two methodologies: a sketch of a comparison}

The generalizations of Kracht's results presented in Sections 5-8 are alternative to those proposed in $[9,10]$, and the aim of the present section is connecting and comparing these two generalizations. Such a comparison is not straightforward, since the methodologies the two generalizations rely on are different: while the treatment in $[9,10]$ relies purely on proof-theoretic notions and is, therefore, internal to proof theory, the present is external, in that is based on a theory (unified correspondence) originating in the model theory of modal logic, developed independently of proof theory, and whose connections with proof theory have not been systematically explored before. As to the basic settings for the two generalizations, the basic setting of the treatment in $[9,10]$ is given by the so called amenable calculi (the definition of which is reported on in Definition 70 below), which are defined for an arbitrary logical signature by means of conditions concerning the performances of the calculus (requiring e.g. that sequents of certain shapes be derivable) rather than the specific shape of the rules of the calculus. For any logical language, and any amenable calculus $\mathcal{C}$, the class of axioms which is proven to give rise to analytic structural rules is defined parametrically in $\mathcal{C}$, as a certain subcollection of the set $\mathcal{I}_{2}(\mathcal{C})$ of those 'formulae $A$ whose logical connectives can be eliminated by applying the invertible logical rules [of $\mathcal{C}$ ] to the premises of those rules obtained by applying some invertible rules to $\mathbf{I} \vdash A$ followed by [the Ackermann lemma]'. The subcollection just mentioned is the one of acyclic formulas, which is defined again taking $\mathcal{C}$ as a parameter. In the present article, the basic environment is given by the class of perfect DLEs, which provides the common semantic environment for both the language of ALBA and for display calculi. In this setting, the logical connectives pertaining to the 'expansion' of the lattice signature are classified into two sets $\mathcal{F}$ and $\mathcal{G}$, according to the order-theoretic properties of their algebraic interpretations. Hence, any DLE-signature is uniquely determined by the sets of logical connectives/function symbols $\mathcal{F}$ and $\mathcal{G}$, which are taken as parameters of the language $\mathcal{L}_{\text {DLE }}=\mathcal{L}_{\text {DLE }}(\mathcal{F}, \mathcal{G})$. The display calculus $\mathbf{D L}$, the language and rules of the appropriate version of ALBA, and the inductive $\mathcal{L}_{\text {DLE}}$-inequalities are then defined parametrically in $\mathcal{F}$ and $\mathcal{G}$ and are hence unique for each choice of $\mathcal{F}$ and $\mathcal{G}$. In Appendix $\mathrm{C}$, we sketch the proof that, for each $\mathcal{F}$ and $\mathcal{G}$, the associated display calculus DL is amenable, and in Appendix D, we show that acyclic inequalities in $\mathcal{I}_{2}(\mathbf{D L})$ can be identified with analytic inductive inequalities.

Notwithstanding their different and mutually independent starting points, once a concrete setting is defined which provides a common ground for the application of the two methodologies, it is not difficult to recognize striking similarities between the algorithm defined in $[9,10]$ for computing analytic structural rules from input analytic inductive inequalities and the ALBA-based procedure illustrated in Section 7.1. In what follows, we are not giving a formal proof establishing systematic connections between the two procedures, and limit ourselves to illustrating them by means of an example.

\subsection{Generalized Church-Rosser inequality}

Let $\mathcal{F}=\{\cdot, \diamond, \triangleleft\}, \mathcal{G}=\{\star, \square, \triangleright\}$, where $\cdot$ and $\star$ are binary and of order type $(1,1), \diamond$ and $\square$ are unary

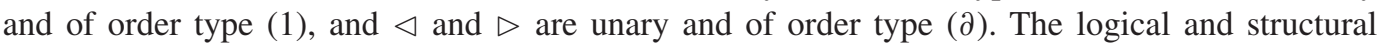


connectives of the display calculi DL and $\mathbf{D L} *$ associated with the basic $\mathcal{L}_{\mathrm{DLE}}(\mathcal{F}, \mathcal{G})$-logic can be represented synoptically as follows (we omit the residuals of the binary connectives since they are not relevant to the present discussion):

\begin{tabular}{|c|c|c|c|c|c|c|c|c|c|c|c|}
\hline I & & () & $\star$ & (8) & (). & (4) & (D) & 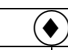 & $(\mathbf{0}$ & 8 & $\bullet$ \\
\hline \begin{tabular}{l|l}
$T$ & $\perp$
\end{tabular} & \begin{tabular}{l|l}
$\wedge$ & $\vee$
\end{tabular} & .1 & \begin{tabular}{l|l} 
& $\star$
\end{tabular} & $\diamond 1$ & 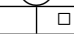 & $\triangleleft 1$ & 18 & $\bullet$ & 10 & 1 & \\
\hline
\end{tabular}

Consider the following analytic inductive inequality:

$$
\square p \cdot \triangleright p \leq \diamond p \star \triangleleft p
$$

Let us implement the procedure illustrated in Section 7.1 on the inequality above:

$$
\begin{array}{ll} 
& \forall p[\square p \cdot \triangleright p \leq \diamond p \star \triangleleft p] \\
\text { iff } & \forall p \forall \vec{q}\left[\left(q_{1} \leq \square p \& q_{2} \leq \triangleright p \& \diamond p \leq q_{3} \& \triangleleft p \leq q_{4}\right) \Rightarrow q_{1} \cdot q_{2} \leq q_{3} \star q_{4}\right] \\
\text { iff } & \forall p \forall \vec{q}\left[\left(\bullet q_{1} \leq p \& q_{2} \leq \triangleright p \& \diamond p \leq q_{3} \& \varangle q_{4} \leq p\right) \Rightarrow q_{1} \cdot q_{2} \leq q_{3} \star q_{4}\right] \\
\text { iff } \quad \forall p \forall \vec{q}\left[\left(\diamond q_{1} \vee \triangleleft q_{4} \leq p \& q_{2} \leq \triangleright p \& \diamond p \leq q_{3}\right) \Rightarrow q_{1} \cdot q_{2} \leq q_{3} \star q_{4}\right] \\
\text { iff } \quad \forall \vec{q}\left[\left(q_{2} \leq \triangleright\left(\diamond q_{1} \vee \triangleleft q_{4}\right) \& \diamond\left(\diamond q_{1} \vee \triangleleft q_{4}\right) \leq q_{3}\right) \Rightarrow q_{1} \cdot q_{2} \leq q_{3} \star q_{4}\right] .
\end{array}
$$

The last quasi-inequality above expresses the validity on perfect DLEs of the following quasi-special structural rule:

$$
\frac{Y \vdash \otimes(\diamond X ; \circlearrowleft W) \diamond(\diamond X ; \diamond W) \vdash Z}{X \odot Y \vdash Z \star W} .
$$

Let us apply the procedure described in $[9,10]$ to the calculus DL and the sequent

$$
\square p \cdot \triangleright p \vdash \diamond p \star \triangleleft p .
$$

We start by exhaustively applying in reverse all invertible rules of DL which are applicable to the sequent. These rules are:

$$
\frac{A \bigodot B \vdash Z}{A \cdot B \vdash Z} \quad \frac{X \vdash A \star B}{X \vdash A \star B} .
$$

This yields the following sequent:

$$
\square p \odot \triangleright p \vdash \diamond p \circledast \triangleleft p .
$$

At this point, the procedure in $[9,10]$ calls for the display of the subformulas on which it is not possible to apply invertible rules as a-parts or s-parts of the premises of the rule-to be. The equivalence of the rule below to the sequent above is guaranteed by the Ackermann lemma:

$$
\frac{X \vdash \square p \quad Y \vdash \triangleright p \quad \diamond p \vdash Z \quad \triangleleft p \vdash W}{X \odot Y \vdash Z \star W} .
$$

On each of the premises of the rule above, more invertible rules of DL can be applied in reverse, namely the following ones:

$$
\frac{X \vdash(0) A}{X \vdash \square A} \quad \frac{X \vdash \otimes A}{X \vdash \triangleright A} \quad \frac{\oslash A \vdash Y}{\diamond A \vdash Y} \quad \frac{\bigotimes A \vdash Y}{\triangleleft A \vdash Y} .
$$

Applying them exhaustively yields

$$
\frac{X \vdash(1) p \quad Y \vdash \otimes p \quad \diamond p \vdash Z \quad \diamond p \vdash W}{X \odot Y \vdash Z \circledast W} .
$$

Modulo replacing $p$ with a fresh structural variable $V$, the rule above satisfies conditions $C_{2}-C_{7}$ but fails to satisfy $C_{1}$. To transform it into an analytic rule, one needs to first display all occurrences of 
the variable $p$, by suitably applying the following display postulates:

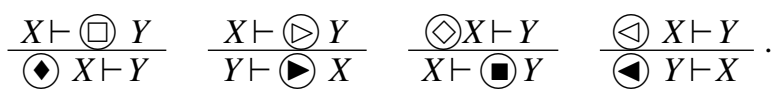

This step yields the following rule:

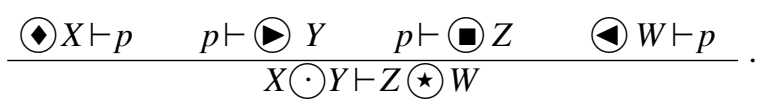

Eliminating $p$ by means of all the possible applications of cut on the premises yields:

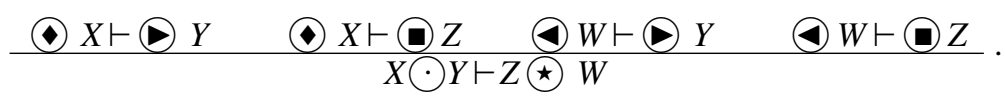

The rule above is analytic and both semantically and DL-equivalent to (9.1). Running the two procedures in parallel shows that they have the same essentials, namely adjunction and Ackermann lemma. Indeed, the cut rules applied on the premises can be assimilated to instances of the Ackermann lemma. Moreover, introduction rules for any given connective are invertible exactly on the side in which the connective is an adjoint/residual. Notice that disjunction (resp. conjunction) is no exception since in the distributive environment it is both a left (resp. right) adjoint and a right (resp. left) residual.

\section{Power and limits of display calculi: conclusion}

The present work addresses the question of which axiomatic extensions of a basic DLE-logic admit a proper display calculus obtained by modularly adding structural rules to the proper display calculus of the basic logic. Such axiomatic extensions are referred to as properly displayable (cf. Definition 27). Our starting point was Kracht's paper [38], which characterizes properly displayable axiomatic extensions of the basic modal/tense logic as those associated with the primitive axioms of the language of classical tense logic. In the present article, we extend Kracht's notion of primitive axiom to primitive inequalities, uniformly defined in any DLE-languages, and prove that Kracht's characterization holds up to semantic equivalence. Specifically, we introduce the class of analytic inductive inequalities as a syntactic extension of primitive inequalities. We show that each analytic inductive inequality can be effectively translated via ALBA into (a set of) analytic rules. In fact, in Section 7, we show that each analytic inductive inequality can be transformed into an analytic rule which is quasispecial (cf. Definition 8). Moreover, in Section 8, we characterize the subclass of analytic inductive inequalities which exactly corresponds to quasi-special rules (cf. Definition 63), and show that each such inequality is in fact frame-equivalent to a primitive inequality. These results, taken together, characterize up to semantic equivalence the properly displayable axiomatic extensions of any basic DLE-logic as as those associated with the primitive inequalities of its associated DLE*-language.

$\underline{\text { Rules }} \quad \underline{\text { Inequalities }}$

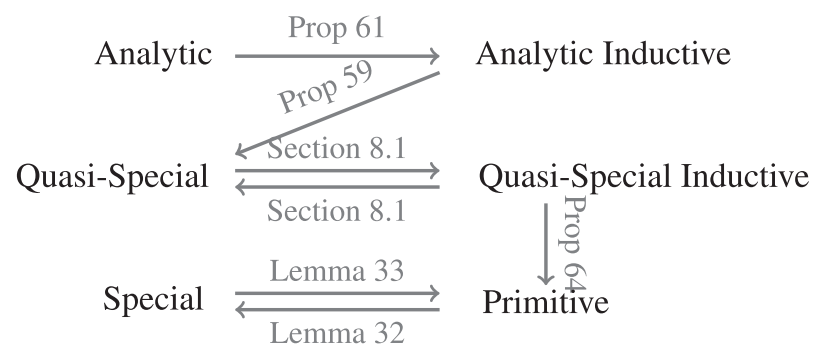


Further applications. The order-theoretic approach to analyticity developed in the present article is applicable also to the display environments for Dynamic Epistemic Logic and PDL developed in $[27,29,30]$, since the order-theoretic properties at the base of the definition of analytic inductive inequalities are available also in those settings. Notice that the settings of [27, 30] are multi-type, i.e. their main feature are logical connectives taking in input terms of possibly different types, which semantically correspond to operations between different algebras. However, the crucial ordertheoretic principles are straightforwardly applicable also to multi-type connectives. To a more limited extent, this approach is also applicable to the settings [4, 28, 31], which do not enjoy the relativized display property. It is worth noticing that the design choices of the calculus introduced in [31] depart from the standard design choices we adopt in the present article. The justification for this non-standard design lies precisely in the fact that, once the axioms of inquisitive logic have been translated into the multi-type environment, one of the axioms can be recognized as not analytic inductive.

\section{Acknowledgements}

The research of the Giuseppe Greco, Alessandra Palmigiano, Apostolos Tzimoulis and Zhiguang Zhao has been made possible by the NWO Vidi grant 016.138 .314 , by the NWO Aspasia grant 015.008.054, and by a Delft Technology Fellowship awarded in 2013. The research of Minghui $\mathrm{Ma}$ is supported by the China national funding of social sciences (grant no.12CZ054). We would like to thank Agata Ciabattoni for inviting three of the authors to a research visit to her group in Vienna. The feedback and questions we received from her and Revantha Ramanayake during this visit significantly improved the paper.

\section{References}

[1] W. Ackermann. Untersuchung über das eliminationsproblem der mathematischen logic. Mathematische Annalen, 110, 390-413, 1935.

[2] N. Belnap. Display logic. Journal of Philosophical Logic, 11, 375-417, 1982.

[3] N. Belnap. Linear logic displayed. Notre Dame Journal of Formal Logic, 31, 14-25, 1990.

[4] M. Bílková, G. Greco, A. Palmigiano, A. Tzimoulis and N. Wijnberg. The logic of resources and capabilities. Submitted for publication.

[5] P. Blackburn, M. de Rijke and Y. Venema. Modal logic. Vol. 53 of Cambridge Tracts in Theoretical Computer Science. Cambridge University Press, 2001.

[6] P. Blackburn, J. van Benthem and F. Wolter, eds. Handbook of Modal Logic. Vol. 3 of Studies in Logic and Practical Reasoning. Elsevier Science, 2006.

[7] A. Ciabattoni, N. Galatos and K. Terui. From axioms to analytic rules in nonclassical logics. In Logic in Computer Science, Vol. 8, pp. 229-240, 2008.

[8] A. Ciabattoni, N. Galatos and K. Terui. Algebraic proof theory for substructural logics: cutelimination and completions. Annals of Pure and Applied Logic, 163, 266-290, 2012.

[9] A. Ciabattoni and R. Ramanayake. Structural extensions of display calculi: a general recipe. In Logic, Language, Information, and Computation: 20th International Workshop WoLLIC 2013, Vol. 8071 of Lecture Notes in Computer Science, pp. 81-95. Springer, 2013.

[10] A. Ciabattoni and R. Ramanayake. Power and limits of structural display rules. ACM Transactions on Computational Logic, 17, 17:1-17:39, 2016.

[11] A. Ciabattoni, L. Straßburger and K. Terui. Expanding the realm of systematic proof theory. In Computer Science Logic, Vol. 5771 of Lecture Notes in Computer Science, pp. 163-178. Springer, 2009. 
[12] W. Conradie and A. Craig. Canonicity results for mu-calculi: an algorithmic approach. Journal of Logic and Computation. In press. ArXiv preprint 1408.6367.

[13] W. Conradie, A. Craig, A. Palmigiano and Z. Zhao. Constructive canonicity for lattice-based fixed point logics. Submitted for publication. ArXiv preprint 1603.06547.

[14] W. Conradie, Y. Fomatati, A. Palmigiano and S. Sourabh. Algorithmic correspondence for intuitionistic modal mu-calculus. Theoretical Computer Science, 564, 30-62, 2015.

[15] W. Conradie, S. Frittella, A. Palmigiano, M. Piazzai, A. Tzimoulis and N. Wijnberg. Categories: how I learned to stop worrying and love two sorts. In Proceedings of WoLLIC 2016. ArXiv preprint 1604.00777.

[16] W. Conradie, S. Ghilardi, and A. Palmigiano. Unified correspondence. In Johan van Benthem on Logic and Information Dynamics, A. Baltag and S. Smets, eds, pp. 933-975. Vol. 5 of Outstanding Contributions to Logic. Springer International Publishing, 2014.

[17] W. Conradie, V. Goranko and D. Vakarelov. Algorithmic correspondence and completeness in modal logic. I. The core algorithm SQEMA. Logical Methods in Computer Science, 2, 1-26, 2006.

[18] W. Conradie and A. Palmigiano. Algorithmic correspondence and canonicity for distributive modal logic. Annals of Pure and Applied Logic, 163, 338-376, 2012.

[19] W. Conradie and A. Palmigiano. Constructive canonicity of inductive inequalities. Submitted for publication. ArXiv preprint 1603.08341.

[20] W. Conradie and A. Palmigiano. Algorithmic correspondence and canonicity for nondistributive logics. Submitted for publication. ArXiv preprint 1603.08515.

[21] W. Conradie, A. Palmigiano and S. Sourabh. Algebraic modal correspondence: Sahlqvist and beyond. Submitted for publication.

[22] W. Conradie, A. Palmigiano, S. Sourabh and Z. Zhao. Canonicity and relativized canonicity via pseudo-correspondence: an application of ALBA. Submitted for publication. ArXiv preprint 1511.04271.

[23] W. Conradie, A. Palmigiano and Z. Zhao. Sahlqvist via translation. Submitted for publication. ArXiv preprint 1603.08220.

[24] W. Conradie and C. Robinson. On Sahlqvist theory for hybrid logics. Journal of Logic and Computation, 2015. doi:10.1093/logcom/exv045.

[25] H. B. Curry. Foundations of Mathematical Logic, McGraw-Hill, 1963.

[26] B. Davey and H. Priestley. Introduction to Lattices and Order. Cambridge University Press, 2002.

[27] S. Frittella, G. Greco, A. Kurz and A. Palmigiano. Multi-type display calculus for propositional dynamic logic. Journal of Logic and Computation, 2014. doi:10.1093/logcom/exu064.

[28] S. Frittella, G. Greco, A. Kurz, A. Palmigiano and V. Sikimic. Multi-type sequent calculi. In Proceedings of Trends in Logic XIII, M. Z. A. Indrzejczak and J. Kaczmarek, eds, pp. 81-93. Łodz University Press, 2014.

[29] S. Frittella, G. Greco, A. Kurz, A. Palmigiano and V. Sikimic. A proof-theoretic semantic analysis of dynamic epistemic logic. Journal of Logic and Computation, 2014. doi:10.1093/logcom/exu063.

[30] S. Frittella, G. Greco, A. Kurz, A. Palmigiano and V. Sikimic. Multi-type display calculus for dynamic epistemic logic. Journal of Logic and Computation, 2014. doi:10.1093/logcom/exu068.

[31] S. Frittella, G. Greco, A. Palmigiano and F. Yang. Structural multi-type sequent calculus for inquisitive logic. In Proceedings of WoLLIC 2016. ArXiv preprint 1604.00936. 
[32] S. Frittella, A. Palmigiano and L. Santocanale. Dual characterizations for finite lattices via correspondence theory for monotone modal logic. Journal of Logic and Computation, 2016. doi:10.1093/logcom/exw011.

[33] M. Gehrke, H. Nagahashi and Y. Venema. A Sahlqvist theorem for distributive modal logic. Annals of Pure and Applied Logic, 131, 65-102, 2005.

[34] R. Goré. Gaggles, Gentzen and Galois: How to display your favourite substructural logic. Logic Journal of the IGPL, 6, 669-694, 1998.

[35] R. Goré. Substructural logics on display. Logic Journal of IGPL, 6, 451-504, 1998.

[36] R. Goré. Dual intuitionistic logic revisited. In TABLEAUX'O0, pp. 252-267, 2000.

[37] R. Goré, L. Postniece and A. F. Tiu. On the correspondence between display postulates and deep inference in nested sequent calculi for tense logics. Logical Methods in Computer Science, 7, 1-38, 2011.

[38] M. Kracht. Power and weakness of the modal display calculus. In Proof Theory of Modal Logic, Applied Logic Series, Vol. 2, pp. 93-121. Kluwer, 1996.

[39] O. Lahav. From frame properties to hypersequent rules in modal logics. In Proceedings of the 2013 28th Annual ACM/IEEE Symposium on Logic in Computer Science, pp. 408-417. IEEE Computer Society, 2013.

[40] B. Lellmann. Axioms vs hypersequent rules with context restrictions: theory and applications. In Automated Reasoning, Vol. 8562 of Lecture Notes in Computer Science, pp. 307-321. Springer, 2014.

[41] B. Lellmann and D. Pattinson. Correspondence between modal Hilbert axioms and sequent rules with an application to S5. In Automated Reasoning with Analytic Tableaux and Related Methods, Vol. 8123 of Lecture Notes in Computer Science, pp. 219-233. Springer, 2013.

[42] M. Ma, A. Palmigiano and M. Sadrzadeh. Algebraic semantics and model completeness for intuitionistic public announcement logic. Annals of Pure and Applied Logic, 165, 963-995, 2014.

[43] S. Marin and L. Straßburger. Label-free modular systems for classical and intuitionistic modal logics. In Advances in Modal Logic 10, Rajeev Goré, Barteld Kooi and Agi Kurucz, eds, pp. 387-406. College Publications, 2014.

[44] S. Negri. Proof analysis in modal logic. Journal of Philosophical Logic, 34, 507-544, 2005.

[45] A. Palmigiano, S. Sourabh and Z. Zhao. Jónsson-style canonicity for ALBA-inequalities. Journal of Logic and Computation, 2015. doi:10.1093/logcom/exv041.

[46] A. Palmigiano, S. Sourabh and Z. Zhao. Sahlqvist theory for impossible worlds. Journal of Logic and Computation, 2016. doi:10.1093/logcom/exw014.

[47] D. R. S. Ramanayake. Cut-Elimination for Provability Logics and Some Results in Display Logic. Australian National University, 2011.

[48] G. Restall. An Introduction to Substructural Logics. Routledge, 2000.

[49] H. Sahlqvist. Completeness and correspondence in the first and second order semantics for modal logic. In Studies in Logic and the Foundations of Mathematics, vol. 82, pp. 110-143. 1975.

[50] P. Schroeder-Heister. Validity concepts in proof-theoretic semantics. Synthese, 148, 525-571, 2006.

[51] P. Schroeder-Heister. Proof-theoretic semantics. In The Stanford Encyclopedia of Philosophy. Summer 2014 edn, E. N. Zalta, ed., 2014.

[52] G. F. Servi. On modal logic with an intuitionistic base. Studia Logica, 36, 141-149, 1977.

[53] G. F. Servi. Semantics for a class of intuitionistic modal calculi. In Italian Studies in the Philosophy of Science, Vol. 47, pp. 59-72. Springer, 1981. 
1434 Unified correspondence as a proof-theoretic tool

[54] V. Sofronie-Stokkermans. Duality and canonical extensions of bounded distributive lattices with operators, and applications to the semantics of non-classical logics I. Studia Logica, 64, 93-132, 2000.

[55] V. Sofronie-Stokkermans. Duality and canonical extensions of bounded distributive lattices with operators, and applications to the semantics of non-classical logics II. Studia Logica, 64, 151-172, 2000.

[56] H. Wansing. Displaying Modal Logic. Kluwer, 1998.

[57] H. Wansing. Sequent systems for modal logics. In Handbook of Philosophical Logic, vol. 8, pp. 61-145, 2002.

Received 10 June 2015

\section{Appendix}

\section{A Cut elimination for the display calculi DL and DL*}

The present appendix focuses on the proof that the calculi DL and DL* defined in Section 4.

\section{FACT 67}

The display calculi DL and $\mathbf{D L}^{*}$ verify condition $\mathrm{C}_{8}$ (cf. Section 2.2).

The reduction step for axioms goes as usual:

$$
\frac{p \vdash p \quad p \vdash p}{p \vdash p} \rightsquigarrow p \vdash p
$$

Now we treat the introductions of the connectives of the propositional base (we also treat here the cases relative to the two additional arrows $\leftarrow$ and $>$ added to our presentation):

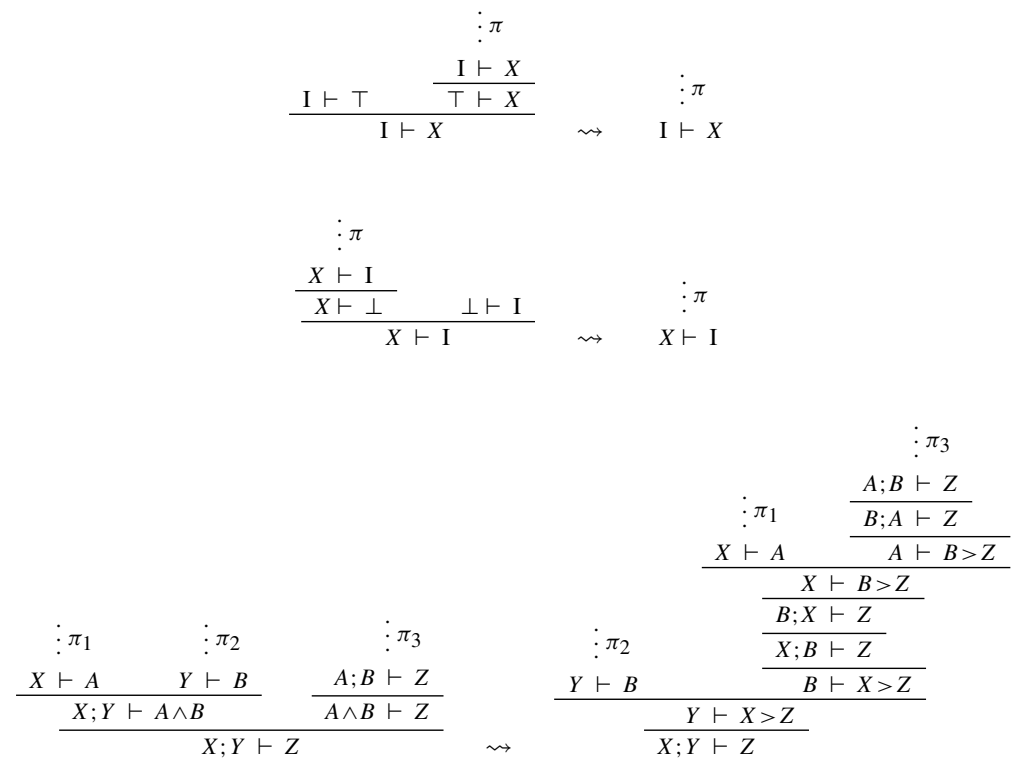




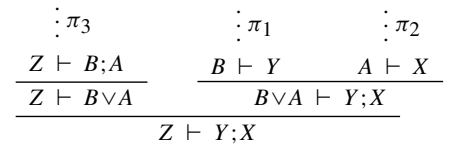

\begin{tabular}{ccc}
$\vdots \pi_{1}$ & $\vdots \pi_{2}$ & $\vdots \pi_{3}$ \\
$\frac{Y \vdash A>B}{Y \vdash A \rightarrow B}$ & $\frac{X \vdash A}{A \rightarrow B} B \vdash Z$ \\
\hline & $Y \vdash X>Z$
\end{tabular}

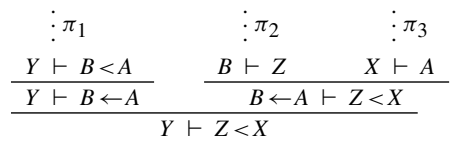

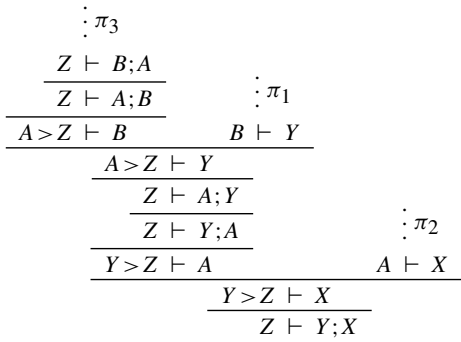
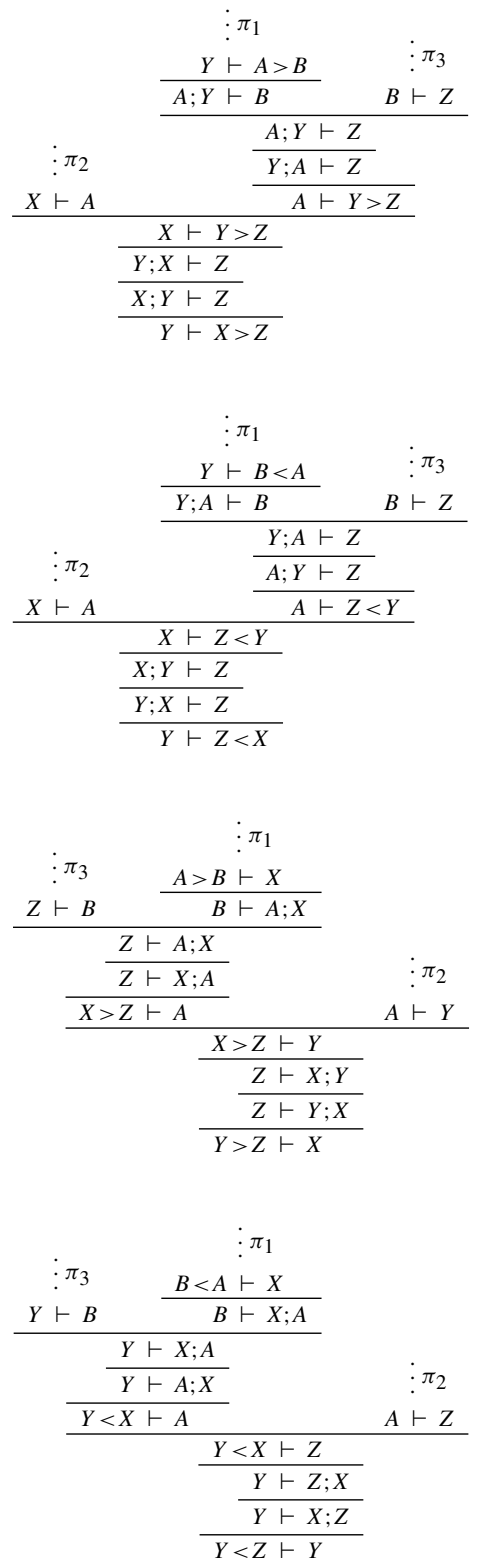


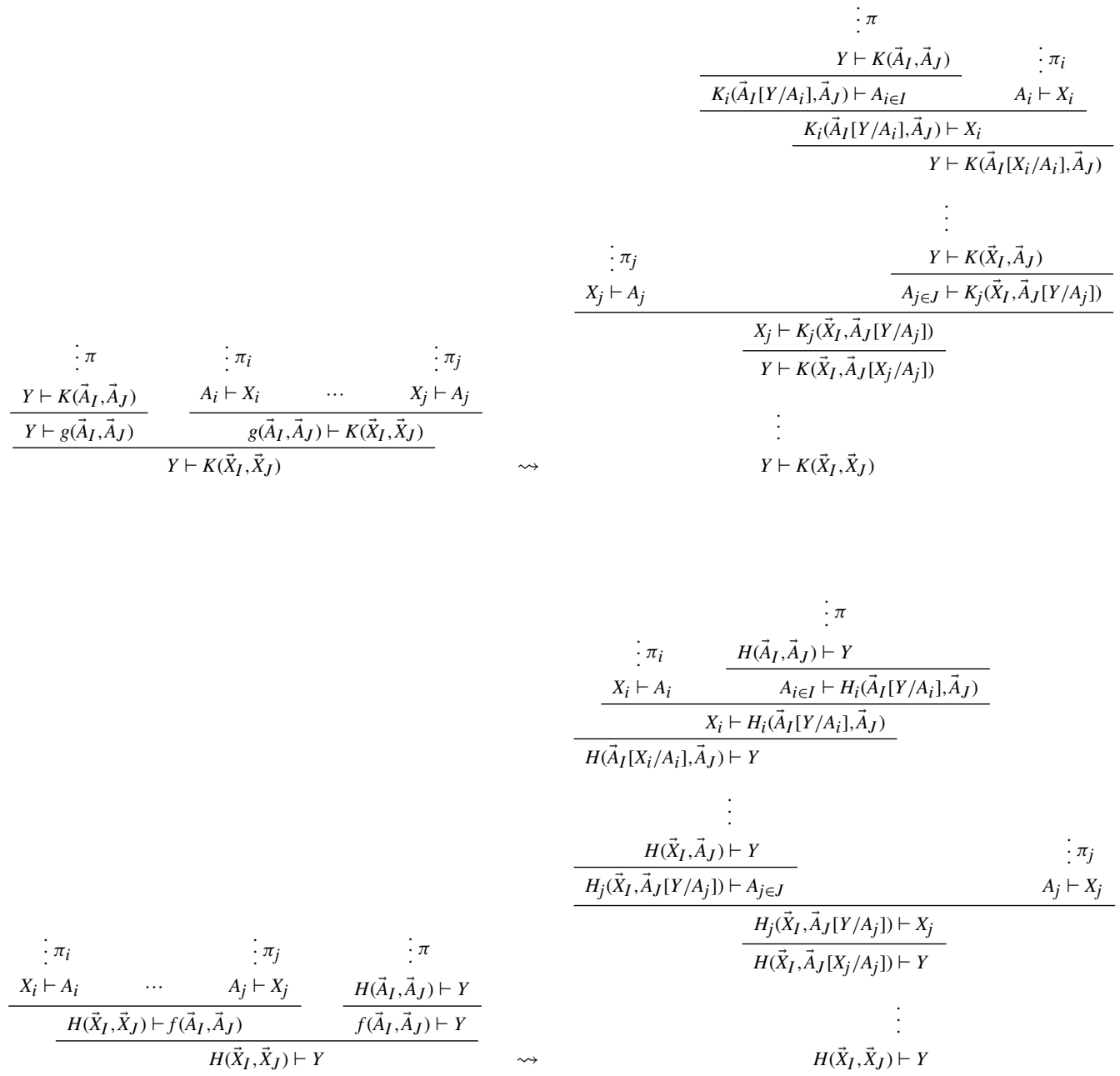

\section{B Invertible rules of DL}

The present appendix characterizes the invertible rules of the calculi DL defined in Section 4. Throughout the present section, fix a language $\mathcal{L}_{\text {DLE }}=\mathcal{L}_{\text {DLE }}(\mathcal{F}, \mathcal{G})$, and let $f \in \mathcal{F}$ and $g \in \mathcal{G}$.

Notice that the following rules are derivable in DL:

$$
\vee_{L^{\prime}} \frac{A \vdash X \quad B \vdash X}{A \vee B \vdash X} \quad \frac{X \vdash A \quad X \vdash B}{X \vdash A \wedge B} \wedge_{R^{\prime}}
$$

Hence, for the sake of the comparison of the two settings, we can add them to DL as primitive rules.

LEMMA 68

The rules $\wedge_{L}, \wedge_{R^{\prime}}, \vee_{R}, \vee_{L^{\prime}}, f_{L}, g_{R}$ are invertible.

ProOF. We only show the cases of $f_{L}$ and $\vee_{L^{\prime}}$, the remaining cases being similar. Assume that $f\left(A_{1}, \ldots, A_{n_{f}}\right) \vdash X$. Then we can derive the premise of $f_{L}$ via the following derivation: 


$$
\frac{\frac{A_{1} \vdash A_{1} \quad \ldots \quad A_{n_{f}} \vdash A_{n_{f}}}{H\left(A_{1}, \ldots, A_{n_{f}}\right) \vdash f\left(A_{1}, \ldots, A_{n_{f}}\right)} \quad f\left(A_{1}, \ldots, A_{n_{f}}\right) \vdash X}{H\left(A_{1}, \ldots, A_{n_{f}}\right) \vdash X .}
$$

Assume $A \vee B \vdash X$. Then we can derive the premises of $\vee_{L^{\prime}}$ via the following derivation:

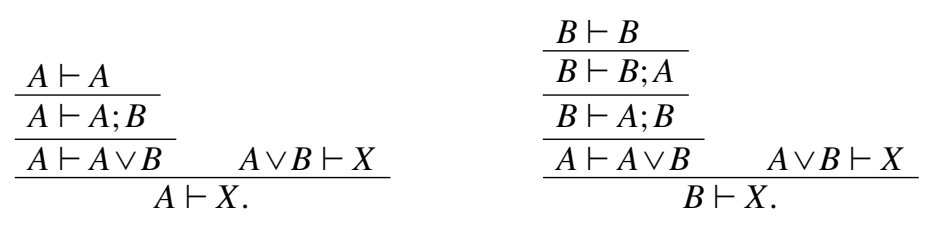

LEMMA 69

The rules $\wedge_{R}, \vee_{L}, f_{R}, g_{L}$ are not invertible.

Proof. Notice that for a rule to be invertible, for each instance of the rule it must be the case that the logical interpretation of each premise is valid in the class of models for DL in which the corresponding conclusion is valid. Hence, to disprove the invertibility of a rule it is enough to find a instance of the rule for which there exists a model satisfying the conclusion but not the premises. We only show this for $f_{R}$, and $\wedge_{R}$, the remaining cases being similar. To show that $f_{R}$ is not invertible, consider the conclusion $H\left(p_{1}, \ldots, p_{n_{f}}\right) \vdash f\left(q_{1}, \ldots, q_{n_{f}}\right)$. Let $\mathbb{A}$ be any Heyting algebra with two incomparable elements $b c$, and let $f^{\mathbb{A}}$ be the $n$-ary operation such that $f(\vec{a})=\perp$ for all $\vec{a} \in \mathbb{A}^{n}$ (notice that this operation is both join-preserving and meet-reversing in each coordinate). Then by letting $v\left(p_{1}\right)=b$ and $v\left(q_{1}\right)=c$, we have that $\perp \leq \perp$ but $b \not \leq c$.

As for $\wedge_{R}$, notice preliminarily that the following instance of the conclusion is derivable:

$$
\frac{A \vdash A \quad B \vdash B}{\frac{A ; B \vdash A \wedge B}{B ; A \vdash A \wedge B}}
$$

Suppose for contradiction that $\wedge_{R}$ was invertible. Then, from $B ; A \vdash A \wedge B$ we would be able to derive both $A \vdash B$ and $B \vdash A$. But since $B ; A \vdash A \wedge B$ is derivable in DL, this would imply that we can also derive $A \vdash B$ for any $A$ and $B$, which contradicts the soundness of the calculus.

\section{The display calculi DL are amenable}

The present appendix sketches the proof that the calculi DL defined in Section 4 are amenable.

DEFINITION 70 (Amenable calculus, cf. [10], Definition 3.1)

Let $\mathcal{C}$ be a display calculus containing an a-structure constant $\mathbf{I}$ and an s-structure constant $\mathbf{I}^{\prime}$ and satisfying $\mathrm{C} 1-\mathrm{C} 8$. Let $\mathfrak{S}(a)$ and $\mathfrak{S}(s)$ denote the class of a- and s-structures of $\mathcal{C}$, and let $\mathcal{L}$ be the language of $L_{\mathbf{I}}(\mathcal{C})$. A display calculus satisfying the following conditions is said to be amenable.

(1) (Interpretation functions) There are functions $l: \mathfrak{S} \mapsto$ For $\mathcal{L}$ and $r: \mathfrak{S} \mapsto$ For $\mathcal{L}$ such that $l(A)=$ $A=r(A)$ for $A \in \operatorname{For} \mathcal{L}$, and for arbitrary $X \in \mathfrak{S}(a)$ and $Y \in \mathfrak{S}(s)$ :

(a) $X \vdash l(X)$ and $Y \vdash l(Y)$ are derivable in $\mathcal{C}$.

(b) If $X \vdash Y$ is derivable in $\mathcal{C}$ then so is $l(X) \vdash r(Y)$.

(2) (Logical constants) There are logical constants $c_{a}, c_{s} \in \operatorname{For}(\mathcal{L})$ such that the following sequents are derivable for arbitrary $X \in \mathfrak{S}(a)$ and $Y \in \mathfrak{S}(s)$ : 


$$
c_{a} \vdash Y \quad X \vdash c_{s}
$$

(3) (Logical connectives) There are binary connectives $\wedge, \vee \in \mathcal{L}$ such that the following sequents are derivable for $\star \in\{\vee, \wedge\}$ :

(a) commutativity: $A \star B \vdash B \star A$

(b) associativity: $A \star(B \star C) \vdash(A \star B) \star C$ and $(A \star B) \star C \vdash A \star(B \star C)$.

Also, for $A, B \in$ For $\mathcal{L}, X \in \mathfrak{S}(a)$ and $Y \in \mathfrak{S}(s)$ :

(a) $\vee A \vdash Y$ and $B \vdash Y$ implies $\vee(A, B) \vdash Y$

(b) $\vee X \vdash A$ implies $X \vdash \vee(A, B)$ for any formula $B$.

(a) $\wedge \quad X \vdash A$ and $X \vdash B$ implies $X \vdash \wedge(A, B)$

(b) $\wedge A \vdash Y$ implies $\wedge(A, B) \vdash Y$ for any formula $B$.

FACT 71

For any $\mathcal{L}_{\mathrm{DLE}}$-language, the corresponding calculus $\mathrm{DL}$ is amenable.

PROOF. The interpretation functions $l$ and $r$ are those defined in Definition 24. The constants are $c_{a}:=\top$ and $c_{s}:=\perp$. Finally, the derivations requested by item (3) are straightforward and omitted.

\section{Analytic inductive inequalities and acyclic $\mathcal{I}_{\mathbf{2}}(\mathrm{DL})$-inequalities}

The following definitions are slight modifications of Definitions 3.7-3.9 in [10]. The modifications essentially amount to specializing the original inequalities from an arbitrary display calculus $\mathcal{C}$ to DL.

\section{DEFINITION 72}

For any sequent $X \vdash Y$ in the language of $\mathbf{D L}$, let $\operatorname{inv}(X \vdash Y)$ denote the collection of sets of sequents obtained by applying sequences of display postulates and invertible logical rules in DL (cf. Appendix B) to it.

\section{DEFINITION 73}

An $\mathcal{L}_{\mathrm{DLE}}$-formula is a-soluble (resp. s-soluble) if there is some $\left\{U_{i} \vdash V_{i} \mid i \in I\right\} \in \operatorname{inv}(s \vdash \mathbf{I})$ (resp. $\in$ $\operatorname{inv}(\mathbf{I}) \vdash s)$ containing no logical connective.

\section{LEMMA 74}

Any $\mathcal{L}_{\text {DLE}}$-formula $s$ is a-soluble (resp. s-soluble) iff $s$ is left-primitive (resp. right-primitive).

PROOF. If $s$ is left-primitive, then every non-leaf node in $+s$ is labelled in one of the following ways: $+f,-g, \pm \wedge$ or $\pm \vee$. Since the left-introduction (resp. right-introduction) rule for any $f \in \mathcal{F}$ (resp. $g \in \mathcal{G}$ ) is invertible and both introduction rules for $\wedge$ and $\vee$ are invertible, a routine induction on the shape of $s$ shows that $s$ is a-soluble. Conversely, if $s$ is not left-primitive, then there exists at least one node in $+s$ which is labelled either $-f$ or $+g$ for some $f \in \mathcal{F}$ or some $g \in \mathcal{G}$. Consider one such node $n$, and let $s^{\prime}$ be the subterm of $s$ rooted at $n$. We can assume w.l.o.g. that all the ancestors of $n$ do not violate the left-primitive requirement. Reasoning like we did before, we can apply suitable invertible rules to all the subformulas of $s$ rooted at the nodes in the path from the root of $+s$ to the direct ancestor of $n$. Then, in the set of sequents obtained as premises of the last rule application, there will be either one sequent of the form $U_{i} \vdash f\left(s_{1}, \ldots, s_{n_{f}}\right.$ ) (if $n$ is labelled $-f$ ) or of the form $g\left(s_{1}, \ldots, s_{n_{g}}\right) \vdash V_{i}$ (if $n$ is labelled $+g$ ). In either case, since the right-introduction (resp. left-introduction) rule for any $f \in \mathcal{F}$ 
(resp. $g \in \mathcal{G}$ ) is not invertible, there is no invertible rule which can be applied to transform the main connective into a structural connective, which proves that $s$ is not a-soluble, as required.

The following definition slightly generalizes the original Definition 3.9 in [10] from formulas to inequalities.

\section{DEFINITION 75}

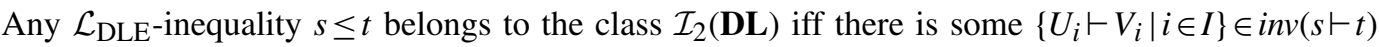
such that, for each $i \in I$, each antecedent-part (resp. succedent-part) formula in $U_{i} \vdash V_{i}$ is s-soluble (resp. a-soluble).

\section{PROPOSITION 76}

The following are equivalent for any $\mathcal{L}_{\mathrm{DLE}}$-inequality $s \leq t$ :

(1) $s \leq t$ belongs to $\mathcal{I}_{2}(\mathbf{D L})$;

(2) every branch in $+s$ and $-t$ is good.

ProOF. By Lemma 74, a term $s$ is left-primitive (resp. right-primitive) if and only if $s$ is a-soluble (ssoluble). Moreover, left-primitive (resp. right-primitive) terms coincide with positive (resp. negative) Skeleton and negative (resp. positive) PIA terms (cf. discussion at the beginning of Section 6.2). If $s \leq t$ is such that every branch is good, then $s \leq t$ is of the form illustrated in the picture below:
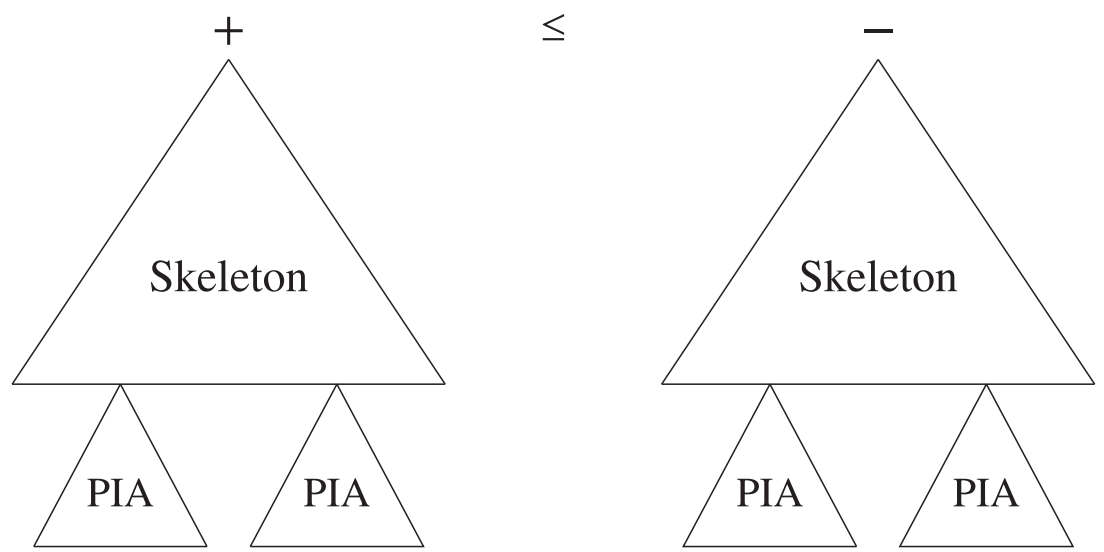

Then it is clear that $s \leq t$ belongs to $\mathcal{I}_{2}(\mathbf{D L})$. Indeed, after applying exhaustively all possible invertible rules to the Skeleton nodes, the PIA parts are 'moved to the premises' via an application of the Ackermann rule, as discussed in Section 9. It is straightforward but tedious to show that, when occurring in the premises, each PIA part is guaranteed to occur on the side on which it is soluble. By definition, this implies that $s \leq t$ is in $\mathcal{I}_{2}(\mathbf{D L})$.

As to the converse direction, notice that each step in the reasoning above can be reversed.

To finish the comparison, we need to report on some definitions from [10]. The following one is a slight modification of [10, Definition 3.18], motivated by the purpose of highlighting its similarity with sets of inequalities in Ackermann shape:

\section{DEFINITION 77}

A nonempty set $\mathcal{S}$ of sequents respects multiplicities w.r.t. a propositional variable $p$ occurring in any of its sequents if $\mathcal{S}$ can be written in one of the following forms via application of display 
rules:

$$
\{p \vdash U \mid p \text { does not occur in } U\} \cup\{S \vdash T \mid p \text { only occurs as s-part in } S \vdash T\}
$$

$\{U \vdash p \mid p$ does not occur in $U\} \cup\{S \vdash T \mid p$ only occurs as a-part in $S \vdash T\}$.

If $\mathcal{S}$ is a set of sequents respecting multiplicities wrt $p$, then $\mathcal{S}$ can be equivalently transformed into the set $\mathcal{S}_{p}$ not containing $p$, and the transformation consists essentially in an application of Ackermann lemma.

Definition 78 (cf. [10, Definition 3.20]) (the set $\mathcal{S}_{p}$ )

Let $\mathcal{S}$ be a set of sequents respecting multiplicities w.r.t. $p$. If $\mathcal{S}$ is uniform in $p$, in the sense that $p$ occurs always as an s-part or an a-part in each sequent of $\mathcal{S}$, then let $\mathcal{S}_{p}:=\{S \vdash T \mid S \vdash T \in \mathcal{S}$ and $p$ does not occur in $S \vdash T\}$. Otherwise, define $\mathcal{S}_{p}$ as the union of $\{S \vdash T \mid S \vdash T \in \mathcal{S}$ and $p$ does not occur in $S \vdash T\}$ and the set of sequents $S^{\prime} \vdash T^{\prime}$ obtained by substituting $p$ for any $U$ such that $p \vdash U$ is in $\mathcal{S}$ (resp. $U \vdash p$ is in $\mathcal{S}$ ) in each sequent $S \vdash T$ in $\mathcal{S}$.

The first case of the definition above corresponds to the situation in which a given variable occurring only positively or negatively is eliminated via Ackermann by suitably replacing it by $\perp$ or $\top$. Clearly, if $\mathcal{S}$ respects multiplicities w.r.t. $p$, then $p$ does not occur in $\mathcal{S}_{p}$ (cf. [10, Lemma 3.21]).

Definition 79 (cf. [10, Definition 3.22]) (acyclic set)

Let $\mathcal{C}$ display calculus. A finite set $\mathcal{S}$ of sequents built from structure variables, structure constants and propositional variables using structural connectives is acyclic if (i) the sequents in $\mathcal{S}$ do not contain any variables; or (ii) there exists a variable $p$ such that $\mathcal{S}$ respects multiplicities w.r.t. $p$ and $\mathcal{S}_{p}$ is acyclic.

\section{LEMMA 80}

Let $\mathcal{S}$ be an acyclic set of sequents in the variables $p_{1}, \ldots, p_{n}$ containing no logical connectives, such that for each variable $p_{i}$ there exist $s_{1}, s_{2} \in \mathcal{S}$ such that $p_{i}$ occurs in antecedent (resp. succedent) position in $s_{1}$ (resp. in $s_{2}$ ). Then there exists a $p$ such that $\mathcal{S}$ can be written in one of the following forms via application of display rules:

$$
\{p \vdash U \mid \text { no logical variable occurs in } U\} \cup\{S \vdash T \mid p \text { only occurs as s-part in } S \vdash T\}
$$

$\{U \vdash p \mid$ no logical variable occurs in $U\} \cup\{S \vdash T \mid p$ only occurs as a-part in $S \vdash T\}$.

ProOF. By induction on the number of variables appearing in $\mathcal{S}$. If it contains only one variable, $p$, then the statement immediately follows from the fact that $\mathcal{S}$ respects multiplicities w.r.t. $p$.

Assume that the statement holds for sets of sequents $\mathcal{S}$ on $n$ variables, and let $\mathcal{S}$ contain $n+1$ variables. Assume for contradiction that the statement is false for each variable $p$ such that $\mathcal{S}$ respects multiplicities w.r.t. $p$. This means that for every such $p$, there is a sequent $p \vdash U$ (or $U \vdash p$ ) as above such that $U$ contains a propositional variable $q$.

Then for every such $p$, the set $\mathcal{S}_{p}$ inherits the same issue: Indeed substituting $U$ for $p$ cannot possibly create terms free from propositional variables, given that $U$ contains $q$. The induction hypothesis implies that each $\mathcal{S}_{p}$ is not acyclic. Then $\mathcal{S}$ is not acyclic, a contradiction.

The following definition is aimed at adapting [10, Definition 3.23] to the setting of DLE-logics. 
DEFINITION 81 (acyclic inequality)

An inequality $s \leq t$ in $\mathcal{I}_{2}(\mathbf{D L})$ is acyclic if there is a set $\left\{\rho_{i}\right\}_{i \in I}$ of semi-structural rules ${ }^{42}$ which is obtained by applying the procedure described in Section 9 to $s \leq t$ such that the set of premises of each $\rho_{i}$ is acyclic.

\section{PROPOSITION 82}

The following are equivalent for any inequality $s \leq t$ :

(1) $s \leq t$ is acyclic and belongs to $\mathcal{I}_{2}(\mathbf{D L})$;

(2) $s \leq t$ is analytic inductive.

Proof. Let $s \leq t$ be analytic $(\Omega, \varepsilon)$-inductive. Then by Proposition $76, s \leq t$ is in $\mathcal{I}_{2}(\mathbf{D L})$. To finish the proof we need to show that it is acyclic. This amounts to proving that the set of premises obtained by applying the Ackermann rule in the procedure described in Section 9 is acyclic. By assumption, $s \leq t$ has the following shape:

$$
\xi_{1}\left(\vec{\phi}_{1} / \vec{x}_{1}, \vec{\psi}_{1} / \vec{y}_{1}, \vec{\gamma}_{1} / \vec{z}_{1}, \vec{\theta}_{1} / \vec{w}_{1}\right) \leq \xi_{2}\left(\vec{\psi}_{2} / \vec{x}_{2}, \vec{\phi}_{2} / \vec{y}_{2}, \vec{\theta}_{2} / \vec{z}_{2}, \vec{\gamma}_{2} / \vec{w}_{2}\right),
$$

where $\xi_{1}\left(\vec{l}_{1}, \overrightarrow{!}_{1}, \vec{l}_{1}, \vec{w}_{1}\right)$ and $\xi_{2}\left(\vec{l}_{2}, \vec{l}_{2}, \vec{l}_{2}, \vec{l}_{2}\right)$, respectively, are a positive and a negative Skeleton formula-cf. page 20, paragraph after Definition 16-(hence $\xi_{1}$ is left-primitive and $\xi_{2}$ is rightprimitive) which are scattered, monotone in $\vec{x}$ and $\vec{z}$ and antitone in $\vec{y}$ and $\vec{w}$. Moreover, the formulas in $\vec{\phi}$ and $\vec{\gamma}$ are positive PIA (and hence right-primitive), and the formulas in $\vec{\psi}$ and $\vec{\theta}$ are negative PIA (and hence left-primitive). Finally, every $\phi$ and $\psi$ contains at least one $\varepsilon$-critical variable, whereas all $+\gamma$ and $-\theta$ are $\varepsilon^{\partial}$-uniform. Without loss of generality we may assume that all formulas in $\vec{\phi}_{i}, \vec{\psi}_{i}$, $\vec{\gamma}_{i}$ and $\vec{\theta}_{i}$ for $i \in\{1,2\}$ are definite PIA (cf. Footnote 29).

Let us apply the procedure described in $[9,10]$ to the calculus DL and the inequality above, seen as a sequent. By exhaustively applying in reverse all invertible rules of $\mathbf{D L}$ which are applicable to the sequent we get the following:

$$
\Xi_{1}\left(\vec{\phi}_{1} / \vec{x}_{1}, \vec{\psi}_{1} / \vec{y}_{1}, \vec{\gamma}_{1} / \vec{z}_{1}, \vec{\theta}_{1} / \vec{w}_{1}\right) \vdash \Xi_{2}\left(\vec{\psi}_{2} / \vec{x}_{2}, \vec{\phi}_{2} / \vec{y}_{2}, \vec{\theta}_{2} / \vec{z}_{2}, \vec{\gamma}_{2} / \vec{w}_{2}\right),
$$

where $\Xi_{1}$ and $\Xi_{2}$ denote the structures associated with $\xi_{1}$ and $\xi_{2}$, respectively. At this point, the procedure in $[9,10]$ calls for the display of the subformulas on which it is not possible to apply invertible rules as a-parts or s-parts of the premises of the rule-to be. The equivalence of the rule below to the sequent above is guaranteed by the Ackermann lemma:

$$
\begin{array}{cccccc}
\vec{X}_{1} \vdash \vec{\phi}_{1} \quad \vec{Y}_{2} \vdash \vec{\phi}_{2} \quad \vec{\psi}_{1} \vdash \vec{Y}_{1} \quad \vec{\psi}_{2} \vdash \vec{X}_{2} \quad \vec{Z}_{1} \vdash \vec{\gamma}_{1} \quad \vec{W}_{2} \vdash \vec{\gamma}_{2} & \vec{\theta}_{1} \vdash \vec{W}_{1} & \vec{\theta}_{2} \vdash \vec{Z}_{2} \\
\hline \Xi_{1}\left(\vec{X}_{1}, \vec{Y}_{1}, \vec{Z}_{1}, \vec{W}_{1}\right) \vdash \Xi_{2}\left(\vec{X}_{2}, \vec{Y}_{2}, \vec{Z}_{2}, \vec{W}_{2}\right)
\end{array}
$$

On each of the premises of the rule above, more invertible rules of DL can be applied in reverse. Applying them exhaustively yields

$$
\begin{gathered}
\vec{X}_{1} \vdash \vec{\Phi}_{1} \quad \vec{Y}_{2} \vdash \vec{\Phi}_{2} \quad \vec{\Psi}_{1} \vdash \vec{Y}_{1} \quad \vec{\Psi}_{2} \vdash \vec{X}_{2} \quad \vec{Z}_{1} \vdash \vec{\Gamma}_{1} \quad \vec{W}_{2} \vdash \vec{\Gamma}_{2} \quad \vec{\Theta}_{1} \vdash \vec{W}_{1} \quad \vec{\Theta}_{2} \vdash \vec{Z}_{2} \\
\hline \Xi_{1}\left(\vec{X}_{1}, \vec{Y}_{1}, \vec{Z}_{1}, \vec{W}_{1}\right) \vdash \Xi_{2}\left(\vec{X}_{2}, \vec{Y}_{2}, \vec{Z}_{2}, \vec{W}_{2}\right)
\end{gathered}
$$

By the definition of inductive inequality, if some $\Omega$-minimal variable occurs in any $\Phi$ or $\Psi$ subterm, then no other variable can occur in that subterm. Hence, the premises of the rule respect multiplicities

\footnotetext{
${ }^{42} \mathrm{~A}$ semi-structural is a rule whose conclusion is constructed from structure variables and structure constants using structural connectives, and whose premises might additionally contain propositional variables.
} 
w.r.t. these variables, which can then be eliminated. Likewise, one can show, by induction on $\Omega$, that all variables can be eliminated, i.e. $s \leq t$ is acyclic, as required.

For the converse direction, assume that $s \leq t$ is acyclic and belongs to $\mathcal{I}_{2}(\mathbf{D L})$. We may assume without loss of generality that all variables in $s \leq t$ occur both positively and negatively, since otherwise they can be eliminated by replacing them with $T$ and $\perp$. By Proposition 76, every branch of the signed generation trees $+s$ and $-t$ is good, and by Definition 81 the following set is acyclic:

$\vec{X}_{1} \vdash \vec{\Phi}_{1} \quad \vec{Y}_{2} \vdash \vec{\Phi}_{2} \quad \vec{\Psi}_{1} \vdash \vec{Y}_{1} \quad \vec{\Psi}_{2} \vdash \vec{X}_{2} \quad \vec{Z}_{1} \vdash \vec{\Gamma}_{1} \quad \vec{W}_{2} \vdash \vec{\Gamma}_{2} \quad \vec{\Theta}_{1} \vdash \vec{W}_{1} \quad \vec{\Theta}_{2} \vdash \vec{Z}_{2}$.

The assumption that each variable occurs both positively and negatively implies that each variable occurs both in antecedent and in consequent position in the sequents above. Hence, by Lemma 80, there exists a propositional variable $p$ such that the above set can be written in one of the following forms via application of display rules:

$$
\begin{gathered}
\{p \vdash U \mid \text { no logical variable occurs in } U\} \cup\{S \vdash T \mid p \text { only occurs as s-part in } S \vdash T\} \\
\{U \vdash p \mid \text { no logical variable occurs in } U\} \cup\{S \vdash T \mid p \text { only occurs as a-part in } S \vdash T\} .
\end{gathered}
$$

Let us define a strict partial order $\Omega$ and an order type $\varepsilon$ on the variables occurring in the set of premises as follows: we declare these $p$ as $\Omega$-minimal elements and we let $\varepsilon(p):=1$ the set of premises is of the second form and $\varepsilon(p):=\partial$ otherwise. Clearly, the set of premises respects multiplicities w.r.t. $p$ which can then be eliminated. In the new set of sequents produced the same reasoning applies. The new variable will be placed above all the $\Omega$-minimal elements. Since the set is acyclic, this process is guaranteed to end after a finite number of rounds, defining an $\varepsilon$ and $\Omega$ for all the variables present. It is routine to check that $s \leq t$ is analytic $(\Omega, \varepsilon)$-inductive. 